\title{
Level raising and symmetric power functoriality, II
}

\author{
Laurent Clozel and Jack A. Thorne*
}

February 24, 2014

\begin{abstract}
We apply automorphy lifting techniques to establish new cases of symmetric power functoriality for Hilbert modular forms of regular algebraic weight. The proof is based on a novel application of an automorphy lifting theorem for residually reducible Galois representations.
\end{abstract}

\section{Contents}

\begin{tabular}{lll}
\hline & Introduction & 2
\end{tabular}

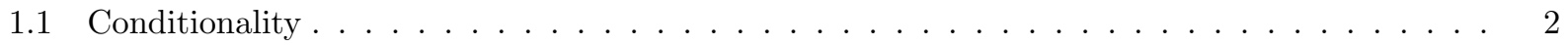

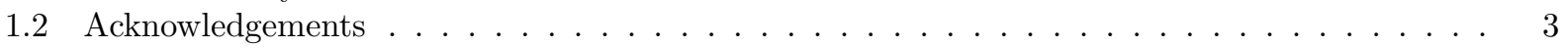

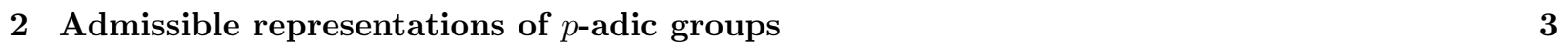

2.1 A ramified unitary group $\ldots \ldots \ldots \ldots \ldots \ldots \ldots \ldots$

2.2 Unitary groups and functoriality $\ldots \ldots \ldots \ldots \ldots \ldots \ldots \ldots$

$\begin{array}{lll}3 & \text { Automorphic representations } & 11\end{array}$

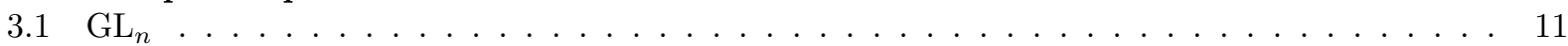

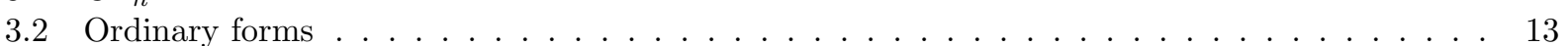

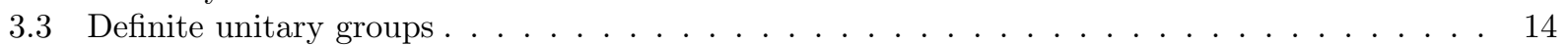

3.4 Endoscopic transfer . . . . . . . . . . . . . . . . . . . . . . . . . 16

3.5 Geometric transfer factors $\ldots \ldots \ldots \ldots \ldots \ldots \ldots \ldots$

3.6 Spectral transfer factors, real places $\ldots \ldots \ldots \ldots \ldots \ldots \ldots$

3.7 Spectral transfer factors, $p$-adic places . . . . . . . . . . . . . . . . . . . . . . . . . 19

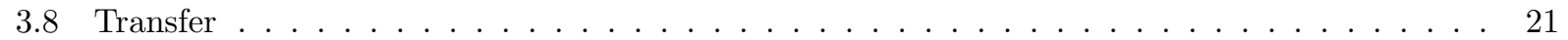

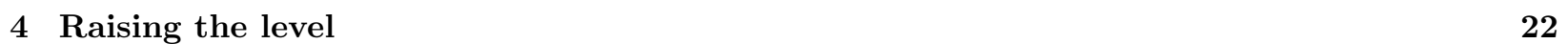

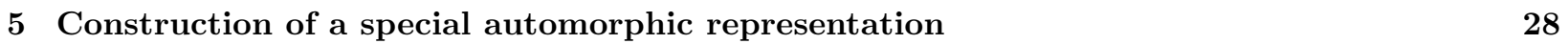

\begin{tabular}{|ll|l}
6 & Proof of Theorem 1.2 & 29
\end{tabular}

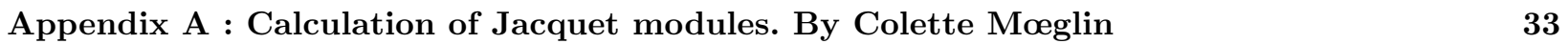

6.1 Le cas quasi déployé, introduction $\ldots \ldots \ldots \ldots \ldots \ldots \ldots \ldots$

$6.2 \quad$ Le cas de $U(n, E), n$ pair et $>4 \ldots \ldots \ldots \ldots \ldots \ldots \ldots \ldots$

\begin{tabular}{ll}
\hline References & 36
\end{tabular}

* Laurent Clozel is a member of Institut Universitaire de France. During the period this research was conducted, Jack Thorne served as a Clay Research Fellow. 


\section{Introduction}

This paper is devoted to the study of a specific instance of Langlands functoriality for $\mathrm{GL}_{2}$. Let us begin with a conjecture. For any notation with which the reader is unfamiliar, we refer to $\$ 3$ below. Let $K$ be a finite Galois extension of the field $\mathbb{Q}$ of rational numbers.

Conjecture 1.1 $\left(\mathrm{SP}_{n+1}(K)\right)$. Let $F$ be a totally real field, linearly disjoint from $K$ over $\mathbb{Q}$. Let $(\pi, \chi)$ be a RAESDC (regular algebraic, essentially self-dual, cuspidal) representation of $\mathrm{GL}_{2}\left(\mathbb{A}_{F}\right)$. Suppose that $\pi$ does not have $C M$, i.e. is not the automorphic induction of an algebraic Hecke character from a quadratic CM extension. Let $n \geq 1$ be an integer.

Then the $n^{\text {th }}$ symmetric power lifting of $\pi$ exists, in the following sense: there exists a RAESDC automorphic representation $(\Pi, \psi)$ of $\mathrm{GL}_{n+1}\left(\mathbb{A}_{F}\right)$ such that for any isomorphism $\iota: \overline{\mathbb{Q}}_{l} \cong \mathbb{C}$, there is an isomorphism of associated Galois representations $\operatorname{Sym}^{n} r_{\iota}(\pi) \cong r_{\iota}(\Pi) \cdot 1^{1}$

Of course this is but a special case of Langlands functoriality; the condition imposed on $F$ of being linearly disjoint from a given field $K$, recurrent since Wiles's work, is due to our deformation-theoretic proofs. (The notation, even if it is clumsy, will alleviate further statements.) Our main result is the following.

Theorem 1.2. Let $l \geq 5$ be a prime. Then the following implication holds:

$$
\mathrm{SP}_{l-1}\left(K\left(\zeta_{l}\right)\right) \Rightarrow \mathrm{SP}_{l+1}\left(K\left(\zeta_{l}\right)\right)
$$

Corollary 1.3. Conjectures $\mathrm{SP}_{6}\left(\mathbb{Q}\left(\zeta_{5}\right)\right)$ and $\mathrm{SP}_{8}\left(\mathbb{Q}\left(\zeta_{35}\right)\right)$ are true.

Proof. Indeed, $\mathrm{SP}_{4}(\mathbb{Q})$ is known to be true, cf. [KS02]. Now use that 5 and 7 are primes.

In the case of elliptic modular forms of weight $k \geq 2$ (thus for $F=\mathbb{Q}$ ), then, the expected tensor products $\mathrm{Sym}^{5}$ and $\mathrm{Sym}^{7}$ exist as cuspidal representations of $\mathrm{GL}_{6}$, resp. GL, over the adèles of $\mathbb{Q}$. For a more ample discussion of Conjecture 1.1, we refer to the paper [CT, of which this one is a sequel. In that paper we outlined a strategy for proving some cases of $\mathrm{SP}_{n+1}(K)$ by reducing it to two other conjectures about automorphic forms, relating to the existence of automorphic tensor products and the construction of so-called 'level-raising' congruences between automorphic representations on unitary groups.

In this paper we carry this strategy out in the first non-trivial case. Namely, we prove a level-raising result for automorphic representations on unitary groups with certain local data, and use this to establish the main theorem above. Our techniques for raising the level seem quite different to previous results in this direction. The problem, and the techniques, go back to Ribet [Rib84]. However we use here an argument introduced by Bellaiche-Graftieaux BG06]. It necessitates the fact that certain representations naturally occurring in our spaces of automorphic forms, and having vectors fixed by the Iwahori subgroup at the "levelraising" prime, have invariants under a larger group. (See Theorem 2.7 as well as the proof of Theorem 4.1.) The fact that suitable representations do occur exactly when needed requires a full understanding of the spaces of automorphic forms on these groups, fortunately now available thanks to the work of Mok [Mok].

\subsection{Conditionality}

Except for some local statements, whose proof is obviously local, in \$2, we warn the reader that most of the proofs in this paper rely on Mok's results [Mok. (For example, note that the simple local statement, Theorem 2.7. clearly depends on a global proof contained in Mok's paper.) We could certainly have dispensed with this, as is done in CHL11. This would, however, considerably obscure the proofs.

The reader is therefore invited to consider the whole paper as conditional on the results in Mok. In turn these results depend on the stabilisation of the trace formula.

Since the case of unitary groups is relatively simple compared with Arthur's proofs for classical groups [Art13, we can state explicitly what is meant by this. As the reader may check, Mok's work becomes unconditional when the following is known.

1. We caution the reader that this conjecture differs slightly in its statement to the conjecture $\operatorname{SP}_{n+1}(\mathbf{K})$ of $[\mathrm{CT}$. 
Mok's proofs necessitate the stabilisation of the trace formula for all quasi-split unitary groups of rank $n, U(n)$ for short, relative to a quadratic extension $E / F$ of number fields; and the stabilisation of the twisted trace formula for $\mathrm{GL}(n, E)$ and the Galois conjugation given by the $F$-form $U(n)$. This is expressed by the identity of traces (3.23) in Arthur's book Art13, expressing the discrete trace of a function $f$ on the adelic points of $U(n)$ or $\mathrm{GL}(n, E)$, as the case may be, as a sum of stable traces. We refer the reader to [Art13, §3.2], for the precise identity, and only note that all the terms are now well-defined due to the proof of the fundamental lemma for units of the Hecke algebra [CL10, CL12, Ngô10, Wal09a, Wal09b] (implying, by Waldspurger's work [Wal97, the corresponding transfer of smooth functions). Moreover, this identity is known to be true in the "non-twisted case", i.e., in our case, for the groups $U(n)$. (As Bergeron, Millson and Moeglin BMM point out, this also assumes a weighted fundamental lemma announced by Chaudouard and Laumon [CL12. See [BMM, Appendix A, footnote 13].) We assume this identity has been proved: this is one of the assumptions in [Mok, Ch. 4]. There has now been considerable progress in this direction, mainly due to Waldspurger and Moeglin. See Wala, Walb.

Given the function $f$, on the adelic points of $U(n)$ or $\operatorname{GL}(n, E)$, the stabilisation is an identity between a certain distribution evaluated at $f$ - the "discrete trace" and a sum of stable distributions evaluated on the images $f^{H}$ of $f$ on (the adelic points of) endoscopic groups. The function $f$, and therefore $f^{H}$, can be assumed to be decomposed. The second assumption in force is the following. Assume $v$ is a finite prime of $F$ and the extension $E / F$ is unramified at $v$. Then the correspondence $f_{v} \leadsto f_{v}^{H}$ of local functions is compatible with the natural map between unramified Hecke algebras given by Langlands functoriality. This implies the properties of the stable expansion formulated as "decomposition by the $\psi$-parameters" used by Mok and formulated in his Lemma 4.3.2. See the discussion in [Art13, §3.3], as well as his Foreword, p. xvii.

\section{$1.2 \quad$ Acknowledgements}

We would first like to thank Colette Mœglin for her help with the proof of Theorem 2.7, and for providing the appendix. We would next like to thank Mark Reeder for making us aware of his papers Ree97, Ree00, and their application to the calculations appearing in the proof of Proposition 2.9. Finally, we thank the anonymous referee for a careful reading of our paper.

\section{Admissible representations of $p$-adic groups}

Let $F$ be a finite extension of $\mathbb{Q}_{p}$, with residue field $k_{F}$, ring of integers $\mathcal{O}_{F}$, uniformizer $\varpi_{F}$, and set $q=\# k_{F}$. In this section we will consider various algebraic $F$-groups $G$. We will abuse notation slightly by writing $G$ both for the group and for its group $G(F)$ of $F$-points. We will use the paper Mor99. as a convenient reference for the facts about Bruhat-Tits theory that we require here; see [Tit79] or [BT72] for more information.

Let $G$ be a connected reductive group over $F$. Let $P=M N$ be a parabolic subgroup of $G$ with Levi subgroup $M$ and unipotent radical $N$, and let $\pi$ be an admissible $\mathbb{C}[G]$-module. The (unnormalized) Jacquet module $\pi_{N}$ of $\pi$ with respect to $N$ is by definition the space of $N$-coinvariants, equipped with its natural $M$-action. We will write $\pi_{N}^{\text {norm }}=\pi_{N} \otimes \delta_{P}^{-1 / 2}$ for the normalized Jacquet module.

Let $S \subset G$ be a maximal $F$-split torus. Associated to the pair $(G, S)$ is the apartment $A=A(G, S)$, affine space under the vector space $V=\left(X^{*}(S) \otimes_{\mathbb{Z}} \mathbb{R}\right)^{*}$. We write $\Phi \subset V^{*}$ for the set of roots with respect to the pair $(G, S)$, and $\Sigma$ for the set of affine roots, which are affine functions on $A$. We fix a choice of Iwahori subgroup $\mathfrak{B} \subset G$. This corresponds to a choice of chamber $C \subset A$, and a set of simple affine roots $\Pi \subset \Sigma$. To a choice of parahoric subgroup $\mathfrak{P}$ containing $\mathfrak{B}$, we can associate a subset $J \subset \Pi$, namely the set of simple affine roots which vanish on the facet $\mathcal{F}$ fixed by $\mathfrak{P}$. We associate to $\mathfrak{P}$ the root subsystem $\Phi_{J}$ of $\Phi$ consisting of the vector parts of the affine roots in $J$.

We associate to $\mathfrak{P}$ a standard Levi subgroup of $G$, as follows. First, let $K \subset G$ denote the reductive subgroup generated by $S$ and the root subgroups $U_{\alpha} \subset G$ for $\alpha$ in the $\mathbb{Z}$-closure of $\Phi_{J}$ inside $\Phi$. Let $Y$ denote the maximal $F$-split torus in the center of $K$. Then the associated Levi subgroup is $M=Z_{G}(Y)$. 
Proposition 2.1. With notation as in the preceding paragraph, let $\pi$ be an admissible representation of $G$, and let $P$ be any parabolic subgroup of $G$ containing $L$ as Levi subgroup. Let $N$ denote the unipotent radical of $P$. Then there is an isomorphism $\pi^{\mathfrak{P}} \cong \pi_{N}^{\mathfrak{P} \cap M}$.

Proof. By [Mor99, Theorem 2.1] and Mor99, Lemma 2.4], $\mathfrak{P}$ has an Iwahori decomposition with respect to $P$, in the sense of [CaS, $\S 1.4]$. Similarly, $\mathfrak{B}$ has an Iwahori decomposition with respect to the minimal parabolic $P_{0}=M_{0} N_{0} \subset G$ containing $Z_{G}(S)$ and contained inside $P$. There is a commutative diagram

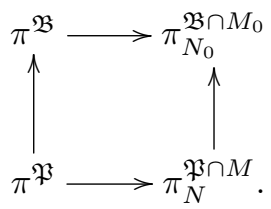

The bottom arrow is surjective, by [Cas, Theorem 3.3.3]. On the other hand, the top arrow is injective, by Mor99, Lemma 3.6]. The commutativity of the diagram now implies that the bottom arrow is also injective, and this completes the proof.

\subsection{A ramified unitary group}

Now suppose that $E / F$ is a ramified quadratic extension, and that the residue characteristic of $F$ is not 2 . In this case we define a unitary group as follows. Let $n=2 m \geq 6$ be an even integer, and define a matrix by

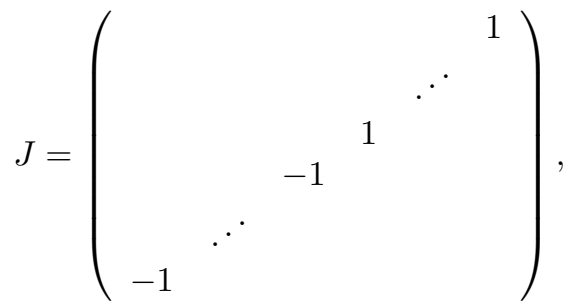

and an $\mathcal{O}_{F}$-group by the formula $\mathcal{G}(R)=\left\{\left.g \in \mathrm{GL}_{n}\left(R \otimes_{\mathcal{O}_{F}} \mathcal{O}_{E}\right)\right|^{t} g J g^{c}=J\right\}$. Then $\mathcal{G}$ is smooth over $\mathcal{O}_{F}$, and its generic fiber $G=U_{n}$ is a quasi-split unitary group in $n$ variables. The group $K=\mathcal{G}\left(\mathcal{O}_{F}\right)$ is a special maximal parahoric subgroup, and there is a surjective map $K \rightarrow \operatorname{Sp}\left(J, k_{F}\right)$ to the symplectic group over the residue field of the symplectic form represented by $J$. The group $\mathcal{G}$ is the integral model of $G$ associated to the maximal parahoric subgroup $K$ as in Tit79, §3.4]. An Iwahori subgroup $\mathfrak{B}$ is the inverse image in $K$ under this map of the subgroup of upper-triangular matrices. A maximal $F$-split torus $S$ of $G$ is the subgroup of matrices of the form

$$
\operatorname{diag}\left(t_{1}, \ldots, t_{m}, t_{m}^{-1}, \ldots, t_{1}^{-1}\right), t_{i} \in F^{\times} .
$$

It naturally extends to an $\mathcal{O}_{F}$-split torus $\mathcal{S} \subset \mathcal{G}$.

Let $\mathcal{P} \subset \mathcal{G}$ denote the subgroup consisting of matrices whose $(i, j)$ entries vanish if $i>j$ and $(i, j) \neq(m+1, m)$. Define $\mathfrak{P}$ to be the parahoric subgroup of $G$ which is the pre-image of $\mathcal{P}\left(k_{F}\right) \subset \operatorname{Sp}\left(J, k_{F}\right)$ inside $K$. The parabolic subgroup associated to $\mathfrak{P}$ by the recipe of the previous section is just $P$, the generic fiber of $\mathcal{P}$. We write $M$ for its standard Levi subgroup, isomorphic to $\left(E^{\times}\right)^{m-1} \times U_{2}$. The base extension of $P$ to $E$ is, under the canonical isomorphism $G(E) \cong \mathrm{GL}_{n}(E)$, the standard parabolic corresponding to the partition

$$
n=\underbrace{1+\cdots+1}_{(n-2) / 2}+2+\underbrace{1+\cdots+1}_{(n-2) / 2} .
$$

Proposition 2.1 now implies the following.

Corollary 2.2. Let $\pi$ be an admissible representation of $G$, and let $P=M N$ denote the Levi decomposition with respect to the maximal $F$-split torus $S$. Then projection induces an isomorphism $\pi^{\mathfrak{P}} \cong \pi_{N}^{\mathfrak{P} \cap M}$. 
Lemma 2.3. The pro-order of $\mathfrak{B}$ is $q^{\infty}(q-1)^{m}$.

Proof. Arguing as in [Tit79, §3.7], we see that the prime-to- $q$ part of the pro-order of $\mathfrak{B}$ is the order of $Z_{\mathcal{G}}(\mathcal{S})\left(k_{F}\right)$, namely $(q-1)^{m}$.

We now introduce the Iwahori-Hecke algebra $H_{\mathfrak{B}}$ of $G$. By definition, this is the convolution algebra of compactly supported $\mathfrak{B}$-biinvariant functions $f: G \rightarrow \mathbb{Z}$. If $R$ is a ring, we write $H_{\mathfrak{B}, R}=H_{\mathfrak{B}} \otimes_{\mathbb{Z}} R$. If $M$ is a smooth $R[G]$-module, then $H_{\mathfrak{B}, R}$ acts on $M^{\mathfrak{B}}$ on the left. The algebra $H_{\mathfrak{B}}$ is non-commutative and has a canonical anti-involution $\jmath$ given on double cosets by $\jmath:[\mathfrak{B} g \mathfrak{B}] \mapsto\left[\mathfrak{B} g^{-1} \mathfrak{B}\right]$. It is useful to recall the following facts.

Proposition 2.4. Let $K$ be a field of characteristic zero.

1. The assignment $\pi \mapsto \pi^{\mathfrak{B}}$ induces an equivalence of categories between the category of admissible $K[G]$ modules which are generated by their $\mathfrak{B}$-invariant vectors and the category of left $H_{\mathfrak{B}, K}$-modules which are finite-dimensional as $K$-vector spaces.

2. Let $\pi$ be an admissible $K[G]$-module which is generated by its $\mathfrak{B}$-invariant vectors. Then $\pi^{\vee}$ corresponds, under the above equivalence, to the module $\operatorname{Hom}_{K}\left(\pi^{\mathfrak{B}}, K\right)$, which we make into a left $H_{\mathfrak{B}, K^{-}}$ module using the anti-involution $\mathrm{J}$.

Proof. These facts are proved in [Bor76] for semisimple $p$-adic groups, but the arguments easily extend to our case.

Let us now say a little more about the structure of the algebra $H_{\mathfrak{B}}$. Fix an element $\varpi \in E$ such that $\varpi^{2} \in F$ is a uniformizer of $F$. With respect to the torus $S$ a choice of set of positive roots is

$$
\left\{t_{i} / t_{j} \mid 1 \leq i<j \leq m\right\} \cup\left\{t_{i} t_{j} \mid 1 \leq i \leq j \leq m\right\} .
$$

The corresponding simple roots are the elements

$$
\alpha_{i}=t_{i} / t_{i+1}, i=1, \ldots, m-1 \text { and } \alpha_{m}=t_{m}^{2} .
$$

This root system is of type $\mathrm{C}_{m}$. We write $W_{0}$ for its Weyl group. If $\alpha \in \Phi$ is a root, we write $s_{\alpha} \in W_{0}$ for the corresponding reflection. We can identify $W_{0} \cong\{ \pm 1\}^{m} \rtimes \mathfrak{S}_{m}$. Here $\mathfrak{S}_{m}$, the symmetric group on the set $\{1, \ldots, m\}$, acts on $S$ by permutation of $t_{1}, \ldots, t_{m}$, and a vector $\mu=\left(\mu_{i}\right)_{i=1}^{m}$ in $\{ \pm 1\}^{m}$ sends $t_{i}$ to $t_{i}^{\mu_{i}}$. We write $w_{0} \in W_{0}$ for the longest element. It is $(-1, \ldots,-1)$, and is central.

Let $Z=Z_{G}(S)$, the maximal torus of $G$ consisting of elements

$$
\operatorname{diag}\left(t_{1}, \ldots, t_{m},{\overline{t_{m}}}^{-1}, \ldots,{\overline{t_{1}}}^{-1}\right), t_{i} \in E^{\times} .
$$

Let $Z_{c} \subset Z$ denote the maximal compact subgroup, and set $\Lambda=Z / Z_{c} \cong \mathbb{Z}^{m}$. A basis of $\Lambda$ is given by the elements

$$
\epsilon_{i}=\operatorname{diag}(1, \ldots, \varpi, \ldots,-1 / \varpi, \ldots, 1), 1 \leq i \leq m,
$$

where $\varpi$ occupies the $i^{\text {th }}$ position. Let $N=N_{G}(S)$. The triple $(G, \mathfrak{B}, N)$ is a generalized Tits system, cf. Cas80, [wa66], and the algebra $H_{\mathfrak{B}}$ admits the following presentation. The extended affine Weyl group $W=\Lambda \rtimes W_{0}$ admits a natural length function $l: W \rightarrow \mathbb{N}$; on the other hand, it has a subgroup, the affine Weyl group $W^{\text {af }} \subset W$ generated by the reflections in the affine roots, cf. [Tit79, §1.7]. We may write $G=\coprod_{w \in W} \mathfrak{B} w \mathfrak{B}$, where the union is disjoint. Writing $G^{0}=\coprod_{w \in W^{\text {af }}} \mathfrak{B} w \mathfrak{B}, G^{0} \subset G$ is a normal subgroup, and $\left(G^{0}, \mathfrak{B}, N \cap G^{0}\right)$ is a Tits system. We write $H^{0} \subset H_{\mathfrak{B}}$ for the subalgebra of elements supported in $G^{0}$.

Let $\Psi \subset W$ denote the subgroup of elements of length zero. There is a decomposition $W=W^{\text {af }} \rtimes \Psi$, and $G / G^{0} \cong \Psi$. In our case, the group $\Psi$ has order two, the non-trivial element being represented by the matrix

$$
\omega=\left(\begin{array}{ccccc}
0 & 0 & \cdots & 0 & 1 / \varpi \\
0 & 1 & \cdots & 0 & 0 \\
\vdots & 0 & \ddots & 0 & \vdots \\
0 & 0 & \cdots & 1 & 0 \\
\varpi & 0 & \cdots & 0 & 0
\end{array}\right) .
$$


It is easy to check that $\omega$ normalizes $\mathfrak{B}$. For each $i=1, \ldots, m$, let $s_{i}=s_{\alpha_{i}}$. Let $s_{0}$ denote the conjugate of $s_{1}$ by $\omega$. Then the elements $s_{0}, \ldots, s_{m} \in W^{\text {af }}$ are the reflections corresponding to the set of simple affine roots induced by $\mathfrak{B}$. Let $B_{W}$ denote the group generated by the elements $T_{w}, w \in W$, subject to

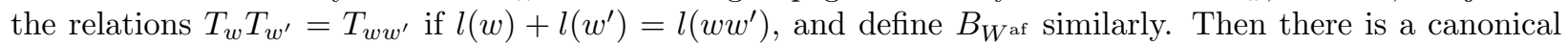
isomorphism between $H_{\mathfrak{B}}$ and the quotient of the group algebra $\mathbb{Z}\left[B_{W}\right]$ by the relations $\left(T_{s_{i}}-1\right)\left(T_{s_{i}}+q\right)=0$, $i=0, \ldots, m$, which takes $\mathfrak{B} w \mathfrak{B}$ to $T_{w}$. Similarly, $H^{0}$ is canonically isomorphic to the quotient of the group algebra $\mathbb{Z}\left[B_{W}\right.$ af $]$ by the same set of relations, and there is an isomorphism

$$
H_{\mathfrak{B}} \cong \mathbb{Z}[\Psi] \widetilde{\otimes} H^{0}
$$

where the twisted tensor product is as in [Iwa66, §5].

We now introduce the Bernstein presentation of the algebra $H_{\mathfrak{B}, \mathbb{C}}$, following Lus89. This is defined in terms of a root system $(X, Y, R, \check{R}, \Pi)$. Here we take $X=\Lambda$ and $Y=\operatorname{Hom}(\Lambda, \mathbb{Z})$. The set $R \subset X$ of roots is taken to consist of the elements

$$
\left\{ \pm \epsilon_{i} \pm \epsilon_{j} \mid 1 \leq i<j \leq m\right\} \cup\left\{ \pm 2 \epsilon_{i} \mid 1 \leq i \leq m\right\}
$$

the simple roots in $\Pi \subset R$ being given by the formulae

$$
\beta_{i}=\epsilon_{i}-\epsilon_{i+1}, 1 \leq i \leq m-1, \beta_{m}=2 \epsilon_{m}
$$

Writing $e_{1}, \ldots, e_{m}$ for the basis of $Y$ dual to $\epsilon_{1}, \ldots, \epsilon_{m}$, the set $\check{R}$ of coroots is

$$
\left\{ \pm e_{i} \pm e_{j} \mid 1 \leq i<j \leq m\right\} \cup\left\{ \pm e_{i} \mid 1 \leq i \leq m\right\}
$$

This root system is isomorphic to that of the group $\mathrm{Sp}_{m}(\mathbb{C})$. It is now easy to check that the extended affine Weyl group defined in Lus89, §1] is just our $W$ above, and the set $S$ of simple reflections constructed there is equal to $\left\{s_{0}, \ldots, s_{m}\right\}$. (The main point to check is as follows. Let $\beta_{0} \in R$ be such that $\check{\beta}_{0} \in \check{R}$ is the lowest root. Then $s_{0}=s_{\beta_{0}} \beta_{0} \in W_{0} \ltimes \Lambda=W$.) Comparing the above discussion with [Lus89, §3] shows that the algebra $H$ constructed by Lusztig in terms of the data $(X, Y, R, \check{R}, \Pi)$ is canonically identified with our algebra $H_{\mathfrak{B}, \mathbb{C}}$, once (in the notation there) $v$ is specialized to $q^{1 / 2}$ and the function $L: S \rightarrow \mathbb{N}$ takes the constant value 1 .

Lusztig defines a presentation, the Bernstein presentation, of the algebra $H_{\mathfrak{B}, \mathbb{C}}$ as a twisted tensor product

$$
H_{\mathfrak{B}, \mathbb{C}} \cong H_{0} \widetilde{\otimes}_{\mathbb{C}} \mathbb{C}[X]
$$

where $H_{0} \subset H_{\mathfrak{B}, \mathbb{C}}$ is the $\mathbb{C}$-subalgebra spanned by the elements $T_{w}, w \in W_{0}$, and $\mathbb{C}[X]$ is the coordinate ring of the complex algebraic torus $\operatorname{Hom}\left(\Lambda, \mathbb{C}^{\times}\right)$. If $\beta \in \Pi$ is a simple root and $s=s_{\beta} \in W_{0}$ is the corresponding simple reflection, then $T_{s} \in H_{0}$ and writing $B_{s}=T_{s}-q$, we have the following relation for all $\theta \in \mathbb{C}[X]$ :

$$
\theta B_{s}=B_{s} \theta^{s}+\left(\theta^{s}-\theta\right) \zeta_{\beta}
$$

where $\zeta_{\beta}=\left(q-e_{\beta}\right) /\left(1-e_{\beta}\right)$. Here we write $e_{\beta} \in \mathbb{C}[X]$ for the element corresponding to $\beta \in X$, and $W_{0}$ acts on $\mathbb{C}[X]$ by its natural right action.

Finally, we relate this presentation to parabolic induction. Let $\tau \in \operatorname{Hom}\left(\Lambda, \mathbb{C}^{\times}\right)$. Then $\tau$ defines a module $\mathbb{C}_{\tau}$ for the group algebra $\mathbb{C}[X]$, which is one-dimensional as $\mathbb{C}$-vector space. Following [Ree97, we define $M(\tau)=H_{\mathfrak{B}, \mathbb{C}} \otimes_{\mathbb{C}[X]} \mathbb{C}_{\tau}$.

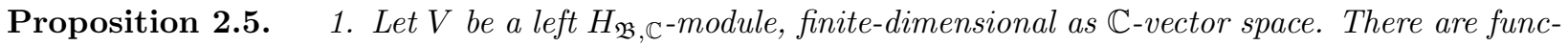
torial isomorphisms

$$
\operatorname{Hom}_{H_{\mathfrak{B}, \mathbb{C}}}(M(\tau), V) \cong \operatorname{Hom}_{\mathbb{C}[X]}\left(\mathbb{C}_{\tau}, V\right) \text { and } \operatorname{Hom}_{H_{\mathfrak{B}, \mathbb{C}}}\left(V, M\left(w_{0} \tau\right)\right) \cong \operatorname{Hom}_{\mathbb{C}[X]}\left(V, \mathbb{C}_{\tau}\right)
$$

2. Let $I(\tau)$ denote the normalized induction of the character $\tau: \Lambda \rightarrow \mathbb{C}^{\times}$, an admissible $\mathbb{C}[G]$-module.

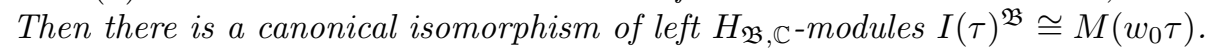


Proof. The first part follows immediately from [Ree97, (3.7)] and the proof of [Ree97, (3.8), Lemma]. For the second part, let $\pi$ be an admissible $\mathbb{C}[G]$-module, generated by its Iwahori-fixed vectors. By Frobenius reciprocity, Cas80, Proposition 2.4], and the first part of the proposition, there are functorial isomorphisms

$$
\operatorname{Hom}_{G}(\pi, I(\tau)) \cong \operatorname{Hom}_{\mathbb{C}[X]}\left(\pi_{N}^{\text {norm }}, \mathbb{C}_{\tau}\right) \cong \operatorname{Hom}_{\mathbb{C}[X]}\left(\pi^{\mathfrak{B}}, \mathbb{C}_{\tau}\right) \cong \operatorname{Hom}_{H_{\mathfrak{B}, \mathbb{C}}}\left(\pi^{\mathfrak{B}}, M\left(w_{0} \tau\right)\right) .
$$

On the other hand, by Proposition 2.4 , there is a functorial isomorphism $\operatorname{Hom}_{G}(\pi, I(\tau)) \cong \operatorname{Hom}_{H_{\mathfrak{B}, \mathbb{C}}}\left(\pi^{\mathfrak{B}}, I(\tau)^{\mathfrak{B}}\right)$. The result now follows from Yoneda's lemma.

\subsection{Unitary groups and functoriality}

Now suppose that $n \geq 6$ is an even integer, and let $E / F$ be a quadratic extension. Let $U_{n}$ denote the quasi-split unitary group in $n$ variables associated to this extension. Let $L_{F}=W_{F} \times \mathrm{SU}_{2}(\mathbb{R})$. The L-group of $U_{n}$ is a semidirect product

$$
{ }^{L} U_{n}=\widehat{G} \rtimes \operatorname{Gal}(E / F)=\mathrm{GL}_{n}(\mathbb{C}) \rtimes \operatorname{Gal}(E / F),
$$

where the non-trivial element $c \in \operatorname{Gal}(E / F)$ acts on $\mathrm{GL}_{n}(\mathbb{C})$ by the automorphism

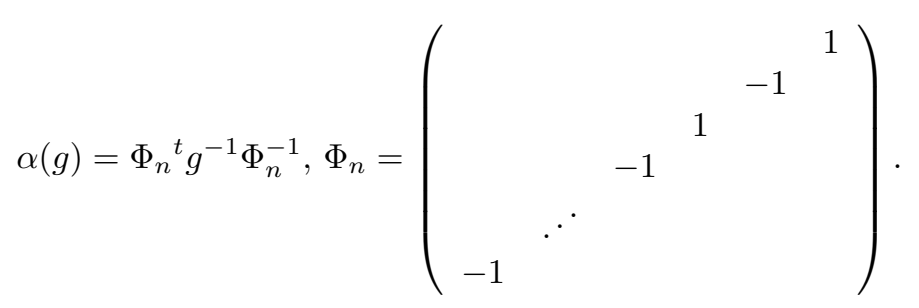

We define an admissible parameter to be a homomorphism $L_{F} \rightarrow{ }^{L} U_{n}$ such that the projection $L_{F} \rightarrow$ ${ }^{L} U_{n} \rightarrow \operatorname{Gal}(E / F)$ is the canonical homomorphism, and $\Phi\left(U_{n}\right)$ to be the set of admissible parameters taken up to $\mathrm{GL}_{n}(\mathbb{C})$-conjugation. If $n=a+b$ is a partition into even integers, then there is an L-homomorphism $\xi:{ }^{L}\left(U_{a} \times U_{b}\right) \rightarrow{ }^{L} U_{n}$, given by formulae

$$
\begin{aligned}
\xi\left(g_{1}, g_{2}, w\right) & =\left(\left(\begin{array}{cc}
g_{1} & 0 \\
0 & g_{2}
\end{array}\right), w\right)\left(w \in W_{E}\right), \\
\xi\left(w_{c}\right) & =\left(\left(\begin{array}{cc}
\Phi_{a} & 0 \\
0 & \Phi_{b}
\end{array}\right) \Phi_{n}^{-1}, w_{c}\right),
\end{aligned}
$$

where $w_{c} \in W_{F} \backslash W_{E}$. On the other hand, there is an injective map $\Phi\left(U_{n}\right) \rightarrow \Phi\left(\mathrm{GL}_{n}(E)\right)$ given by restriction of parameters to $L_{E}$. If $G=U_{n}$ or $\mathrm{GL}_{n}(E)$ we write $\Phi_{\text {bdd }}(G)$ for the subset of parameters $\varphi$ such that $\varphi\left(W_{E}\right)$ is a bounded subset of $\widehat{G}$, and $\Pi_{\text {temp }}(G)$ for the set of isomorphism classes of irreducible admissible representations of $G$ which are tempered.

Lemma 2.6. This map induces a bijection between $\Phi\left(U_{n}\right)$ and the subset of $\Phi\left(\mathrm{GL}_{n}(E)\right)$ consisting of those parameters which are conjugate symplectic, in the sense of [Mok, §2.2].

Proof. This follows from $\mathrm{Mok}$, Lemma 2.2.1]. It uses that $n$ is even.

Given $\varphi \in \Phi(G)$ we define groups

$$
S_{\varphi}=Z_{\widehat{G}}(\operatorname{im} \varphi), \bar{S}_{\varphi}=S_{\varphi} / Z(\widehat{G})^{\Gamma_{F}}, \mathcal{S}_{\varphi}=\pi_{0}\left(\bar{S}_{\varphi}\right) .
$$

In [Mok, Theorem 2.5.1] is associated to each $\varphi \in \Phi_{\mathrm{bdd}}\left(U_{n}\right)$ a finite set $\Pi_{\varphi}$ of isomorphism classes of tempered irreducible admissible representations of $U_{n}$, and a bijective mapping $\Pi_{\varphi} \rightarrow \operatorname{Hom}\left(\mathcal{S}_{\varphi}, \mathbb{C}^{\times}\right)$. This set is characterized by certain character identities. The set $\Pi_{\text {temp }}\left(U_{n}\right)$ is the disjoint union of the sets $\Pi_{\varphi}$ for $\varphi \in \Phi_{\mathrm{bdd}}\left(U_{n}\right)$. We refer to $\Pi_{\varphi}$ as the L-packet associated to $\varphi$. If $\pi \in \Pi_{\varphi}$ and $\varphi$ is a bounded parameter, 
then we define the stable base change $\mathrm{BC}(\pi)$ to be the irreducible admissible representation of $\mathrm{GL}_{n}(E)$ corresponding to the restriction of $\varphi$ in $\Phi_{\mathrm{bdd}}\left(\mathrm{GL}_{n}(E)\right)$.

We will be in interested in a particular L-packet. Suppose once more that $E / F$ is ramified, and that the residue characteristic of $F$ is not 2. We write $\mathrm{St}_{n, E}$ for the Steinberg representation of $\mathrm{GL}_{n}(E)$. The following description of our L-packet of interest was explained to us by Moeglin, who has kindly written up a proof in the appendix to this paper. Recall that there is a correspondence $f_{E} \leadsto f$ between compactly supported, smooth functions on $\mathrm{GL}_{n}(E)$ and $U_{n}$. This is a correspondence, not a map; we will simply say that $f_{E}$ and $f$, or equivalently $f$ and $f_{E}$, are associated.

Theorem 2.7. The representation $\Pi_{E}=\mathrm{St}_{2, E} \boxplus \mathrm{St}_{n-2, E}$ of $\mathrm{GL}_{n}(E)$ is in the image of the stable base change map. The corresponding L-packet of $U_{n}$ contains exactly two elements $X, Y$ which may be characterized as follows.

$$
\begin{aligned}
- & \langle\operatorname{tr} X+\operatorname{tr} Y, f\rangle=\left\langle\operatorname{tr} \Pi_{E} \times I_{c}, f_{E}\right\rangle, \text { where the intertwining operator } I_{c}: \Pi_{E} \cong \Pi_{E}^{c} \text { is Whittaker } \\
& \text { normalized, cf. } \sqrt{3.7} \text { below. } \\
- & \operatorname{dim} X^{\mathfrak{P}}=\operatorname{dim} X^{\mathfrak{B}}=1 \text { and } \operatorname{dim} Y^{\mathfrak{B}}=n / 2+1 . \\
- & X_{N_{0}}^{\text {norm }}=[n-3, n-5, \ldots, 1,-1] \text { and }\left(Y_{N_{0}}^{\text {norm }}\right)^{s s}=[1, n-3, n-5, \ldots, 1]+\sum_{i=1}^{n / 2-3}[n-3, \ldots, n- \\
& 1-2 i, 1, n-3-2 i, \ldots, 1]+2[n-3, n-5, \ldots, 1,1]+[n-3, n-5, \ldots, 1,-1] .
\end{aligned}
$$

Here we write $\left[a_{1}, \ldots, a_{n / 2}\right]$ for the character $|\cdot|^{a_{1} / 2} \otimes \cdots \otimes|\cdot|^{a_{n / 2} / 2}$ of $E^{\times} \times \cdots \times E^{\times}$, the $F$ points of the standard Levi subgroup of the minimal parabolic $P_{0} \subset U_{n}$. We remark that the character $[n-3, n-5, \ldots, 1,1]$ occurs in $\left(Y_{N_{0}}^{\text {norm }}\right)^{\mathrm{ss}}$ with multiplicity two, while every other character occurs with multiplicity one.

Proof. Mok's results imply the existence of two irreducible modules $X^{\prime}$ and $Y^{\prime}$ satisfying the first equality in the theorem, cf. Mok, Theorem 3.2.1]. On the other hand, Moeglin shows the existence of the same equality for the modules $X, Y$ described in the Appendix, $\langle\operatorname{tr} X+\operatorname{tr} Y, f\rangle$ being replaced by $\langle a \operatorname{tr} X+b \operatorname{tr} Y, f\rangle$ with $a, b \neq 0$ [Mœg07, Proposition 5.5]. On the elements of a maximally split torus $T_{0}$ of $U_{n}$ and the associated elements of $\mathrm{GL}_{n}(E)$, the correspondence is a simple identity of orbital integrals (twisted for $f_{E}$ ). In particular, the orbital integrals of $f$ are arbitrary, say on regular elements of $T_{0}$, for $f$ in the image of the correspondence. It follows that $a=b=1$; the Jacquet modules of $X+Y, X^{\prime}+Y^{\prime}$ are then determined by this identity (by Casselman's theorem: see [Clo90a, (2.4)]). It is now easy to check that $X, Y$ and $X^{\prime}, Y^{\prime}$ coincide, up to reordering. The second and third assertions of the theorem now follow from the Appendix, except for the statement on $X^{\mathfrak{P}}$.

By Proposition 2.1 it suffices to check that $X_{N}^{\mathfrak{B} \cap M} \neq 0$. By Frobenius reciprocity and transitivity of the Jacquet modules, there exists a non-trivial map from $X_{N}$ to $[n-3, \ldots, 1] \otimes \operatorname{Ind}_{P_{0}^{2}}^{U_{2}}|\cdot|^{-1 / 2}, P_{0}^{2}$ being the Borel subgroup in $U_{2}$. The induced representation has the trivial representation as its only submodule.

Proposition 2.8. Let $\varphi: H_{\mathfrak{B}} \rightarrow \mathbb{C}$ denote the homomorphism giving the action of $H_{\mathfrak{B}}$ on $X^{\mathfrak{B}}$. Then $\varphi \circ \jmath=\varphi$.

Proof. This is an immediate consequence of the fact that $X$ is self-dual, which may be checked as follows. Given the structure of the Jacquet modules of $X$ and $Y$, it suffices to show that $X+Y$ is self-dual in the Grothendieck group of admissible $\mathbb{C}\left[U_{n}\right]$-modules. The correspondence $f_{E} \leadsto f$ is compatible with the antiinvolutions $g \mapsto g^{-1}$ (on both groups), so it suffices to check that there is an isomorphism $\Pi_{E} \cong \Pi_{E}^{\vee}$, compatible with the Whittaker functional. However $\Pi_{E}^{\vee}$ is isomorphic to the representation $\Pi_{E}\left(\Phi_{n}{ }^{t} g^{-1} \Phi_{n}^{-1}\right)=\rho_{E}$, say, and an isomorphism $\Pi_{E} \rightarrow \rho_{E}$ respects the Whittaker functional.

Fix an odd prime $l$ and an isomorphism $\iota: \overline{\mathbb{Q}}_{l} \cong \mathbb{C}$, and let $K \subset \overline{\mathbb{Q}}_{l}$ be a finite extension of $\mathbb{Q}_{l}$, with ring of integers $\mathcal{O}$ and residue field $k$. We suppose that $K$ contains a square root of $q$.

Proposition 2.9. $\quad$ 1. $\iota^{-1} X$ and $\iota^{-1} Y$ are defined over $K$. We write $X_{K}, Y_{K}$ for a choice of admissible $K\left[U_{n}\right]$-modules satisfying

$$
X_{K} \otimes_{K, \iota} \mathbb{C} \cong X, Y_{K} \otimes_{K, \iota} \mathbb{C} \cong Y
$$


2. Suppose that $l \nmid q(q+1) \prod_{i=1}^{n / 2-2}\left(q^{i}-1\right)$. Then there exist $H_{\mathfrak{B}, \mathcal{O}}$-submodules $X_{\mathcal{O}}^{\mathfrak{B}} \subset X_{K}^{\mathfrak{B}}$ and $Y_{\mathcal{O}}^{\mathfrak{B}} \subset Y_{K}^{\mathfrak{B}}$ such that the natural maps

$$
X_{\mathcal{O}}^{\mathfrak{B}} \otimes_{\mathcal{O}} K \rightarrow X_{K}^{\mathfrak{B}} \text { and } Y_{\mathcal{O}}^{\mathfrak{B}} \otimes_{\mathcal{O}} K \rightarrow Y_{K}^{\mathfrak{B}}
$$

are isomorphisms, and $X_{\mathcal{O}}^{\mathfrak{B}} \otimes_{\mathcal{O}} k$ and $Y_{\mathcal{O}}^{\mathfrak{B}} \otimes_{\mathcal{O}} k$ have no Jordan-Hölder factors as $H_{\mathfrak{B}, k \text {-modules in }}$ common.

Proof. We give a proof by explicit calculation, using the results of Reeder [Ree97, Ree00. We use the notation for the algebra $H_{\mathfrak{B}}$ established in the previous section. If $M$ is a $\mathbb{C}[\Lambda]$-module and $\tau: \Lambda \rightarrow \mathbb{C}^{\times}$is a homomorphism, we write $M\left[\tau^{\infty}\right]$ for the subspace which is annihilated by some power of the ideal $\mathfrak{m}_{\tau} \subset \mathbb{C}[\Lambda]$, kernel of the associated homomorphism $\mathbb{C}[\Lambda] \rightarrow \mathbb{C}$.

Let $\tau_{0}$ denote the character $[n-3, n-5, \ldots, 1,1]$ of $\Lambda$. As observed above, $Y^{\mathfrak{B}}\left[\tau_{0}^{\infty}\right]$ has dimension

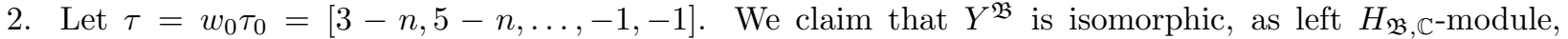
to the submodule of $M(\tau)$ generated by $M(\tau)\left[\tau_{0}^{\infty}\right]$. Indeed, by Proposition 2.5, there is an injection of

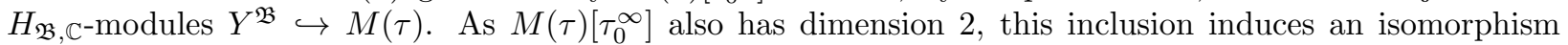
$Y^{\mathfrak{B}}\left[\tau_{0}^{\infty}\right] \cong M(\tau)\left[\tau_{0}^{\infty}\right]$, implying the claim. We now use this to compute a model for $Y^{\mathfrak{B}}$, and then calculate its reduction modulo $l$.

The non-trivial characters of $\Lambda$ occurring in $Y^{\mathfrak{B}}$ are $\tau_{0}, s_{m} \tau_{0}$, and $s_{m-2} \tau_{0}, s_{m-3} s_{m-2} \tau_{0}, \ldots$, $s_{1} \ldots s_{m-3} s_{m-2} \tau_{0}$. Using Ree00, Proposition 2.1], we can calculate bases for the weight spaces of these characters in $Y^{\mathfrak{B}}$ and the matrices of the operators $T_{s_{i}}$. Let us treat first the case $n=6, \tau_{0}=[3,1,1]$. (The module $Y^{\mathfrak{B}}$ then corresponds to the module $V_{01}$ of [Ree97, $\left.\S 13.2\right]$; note that our [3,1,1] is Reeder's $[-3,-1,-1]$.) The stabilizer of $\tau$ in $W_{0}$ is $W_{0, \tau}=\left\{1, s_{m-1}\right\}=\left\{1, s_{2}\right\}$. One calculates using Ree00, Proposition 2.1] and [Ree00, Proposition 2.3] that a basis for $Y^{\mathfrak{B}} \subset M(\tau)$ is given by the vectors (Reeder's notation)

$$
\left\{H_{s_{m} w_{0} s_{m-1}} \otimes 1, H_{w_{0}} \otimes 1, H_{w_{0} s_{m-1}} \otimes 1, H_{s_{m-2} w_{0} s_{m-1}} \otimes 1\right\}=\left\{H_{s_{3} w_{0} s_{2}} \otimes 1, H_{w_{0}} \otimes 1, H_{w_{0} s_{2}} \otimes 1, H_{s_{1} w_{0} s_{2}} \otimes 1\right\} .
$$

With respect to this basis, the operators $T_{s_{i}}$ are given by the matrices

$$
\begin{aligned}
T_{s_{1}} & =\left(\begin{array}{cccc}
-1 & 0 & 0 & 0 \\
0 & -1 & 0 & 0 \\
0 & q & -1 & 1 \\
0 & q(q+1) & 0 & q
\end{array}\right), \\
T_{s_{2}} & =\left(\begin{array}{cccc}
-1 & 0 & 0 & 0 \\
0 & -1 & 1 & 0 \\
0 & 0 & q & 0 \\
0 & 0 & 0 & -1
\end{array}\right), \\
T_{s_{3}} & =\left(\begin{array}{cccc}
q & 2 q(q+1) & 0 & 0 \\
0 & -1 & 0 & 0 \\
1 & 2 q & -1 & 0 \\
0 & 0 & 0 & -1
\end{array}\right) .
\end{aligned}
$$

The group $\Lambda$ is freely generated by the elements $\epsilon_{i}, i=1,2,3$, and these elements act on $Y^{\mathfrak{B}}$ by the matrices

$$
\begin{gathered}
\epsilon_{1}=\left(\begin{array}{cccc}
q^{-3 / 2} & 0 & 0 & 0 \\
0 & q^{-3 / 2} & 0 & 0 \\
0 & 0 & q^{-3 / 2} & 0 \\
0 & 0 & 0 & q^{-1 / 2}
\end{array}\right), \\
\epsilon_{2}=\left(\begin{array}{cccc}
q^{-1 / 2} & 0 & 0 & 0 \\
0 & q^{-1 / 2} & 0 & 0 \\
0 & q^{1 / 2}-q^{-1 / 2} & q^{-1 / 2} & 0 \\
0 & 0 & 0 & q^{-3 / 2}
\end{array}\right),
\end{gathered}
$$




$$
\epsilon_{3}=\left(\begin{array}{cccc}
q^{1 / 2} & 0 & 0 & 0 \\
0 & q^{-1 / 2} & 0 & 0 \\
0 & q^{-1 / 2}-q^{1 / 2} & q^{-1 / 2} & 0 \\
0 & 0 & 0 & q^{-1 / 2}
\end{array}\right)
$$

(We remark that $q^{1 / 2}$ is a canonically defined element of $\mathbb{R} \subset \mathbb{C}$.) Let $Y_{\mathbb{Z}\left[q^{1 / 2}, q^{-1 / 2}\right]}$ denote the free $\mathbb{Z}\left[q^{1 / 2}, q^{-1 / 2}\right]$-module spanned by the above basis elements. Then

$$
Y_{\mathbb{Z}\left[q^{1 / 2}, q^{-1 / 2}\right]}^{\mathfrak{B}} \subset Y^{\mathfrak{B}}
$$

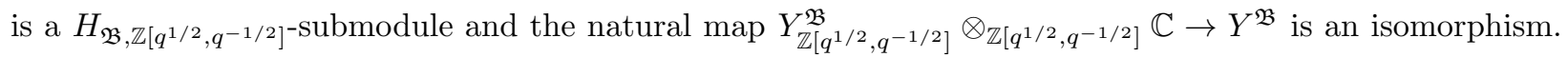
The choice of $\iota$ induces a homomorphism $\mathbb{Z}\left[q^{1 / 2}, q^{-1 / 2}\right] \rightarrow \mathcal{O}$. We set

$$
Y_{\mathcal{O}}^{\mathfrak{B}}=Y_{\mathbb{Z}\left[q^{1 / 2}, q^{-1 / 2}\right]}^{\mathfrak{B}} \otimes_{\mathbb{Z}\left[q^{1 / 2}, q^{-1 / 2}\right]} \mathcal{O},
$$

and choose $X_{\mathcal{O}}^{\mathfrak{B}} \subset X_{K}^{\mathfrak{B}}$ to be any $\mathcal{O}$-submodule of rank 1 . The proposition now follows in this case from the fact that the above matrices generate, after reduction $\bmod \lambda$, the whole algebra $\operatorname{End}_{k}\left(Y_{\mathcal{O}}^{\mathfrak{B}} \otimes_{\mathcal{O}} k\right)=M_{4}(k)$. Indeed, it is easy to see that the matrices $\epsilon_{1}, \epsilon_{2}, \epsilon_{2}$ generate a subalgebra of $M_{4}(k)$ containing the diagonal matrices $\operatorname{diag}(\lambda, \mu, \mu, \nu), \lambda, \mu, \nu \in k$. Multiplying the matrices $T_{s_{1}}, T_{s_{2}}$ and $T_{s_{3}}$ on the left and right by matrices of this form, and using that $2 q\left(q^{2}-1\right)$ is non-zero in $k$, we obtain the elementary matrices $E_{3,4}$, $E_{4,3}, E_{1,2}$ and $E_{3,1}$, where $E_{i, j}$ is the matrix with exactly one non-zero entry in the $(i, j)$ spot, which is equal to 1 . Using the matrices $\epsilon_{2}$ and $T_{s_{2}}$, we obtain all block diagonal matrices with blocks of size $1+2+1=4$. It is now easy to check that the algebra generated by all of these operators is $M_{4}(k)$.

We treat the general case by induction on $n \geq 8$. Suppose the proposition to be true for the group

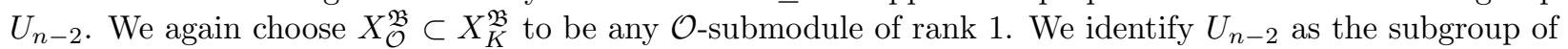
$U_{n}$ consisting of block diagonal matrices, corresponding to the partition $n=1+(n-2)+1$. We write $Y_{n-2}$ for the corresponding representation of $U_{n-2}$. Similarly we write $\mathfrak{B}_{n-2} \subset U_{n-2}$ for the Iwahori subgroup of this group. We can view $H_{\mathfrak{B}_{n-2}, \mathbb{C}}$ as a subalgebra of $H_{\mathfrak{B}, \mathbb{C}}$, namely the one generated by the elements $T_{s_{2}}, \ldots, T_{s_{m}} \in H_{0}$ and $\epsilon_{2}^{ \pm 1}, \ldots, \epsilon_{m}^{ \pm 1} \in \mathbb{C}[\Lambda]$. One calculates using [Ree00, Proposition 2.3] that a basis for $Y^{\mathfrak{B}}$ is given by the elements

$$
\left\{H_{s_{m} w_{0} s_{m-1}} \otimes 1, H_{w_{0}} \otimes 1, H_{w_{0} s_{m-1}} \otimes 1, H_{s_{m-2} w_{0} s_{m-1}} \otimes 1, H_{s_{m-3} s_{m-2} w_{0} s_{m-1}} \otimes 1, \ldots, H_{s_{1} \ldots s_{m-3} s_{m-2} w_{0} s_{m-1}} \otimes 1\right\} .
$$

We first show that the $\mathcal{O}$-submodule $Y_{\mathcal{O}}^{\mathfrak{B}}$ of $\iota^{-1} Y^{\mathfrak{B}}$ spanned by these elements is $H_{\mathfrak{B}, \mathcal{O}}$-invariant. Indeed, the $\mathcal{O}$-submodule spanned by the first $m$ of these elements is preserved by the subalgebra $H_{\mathfrak{B}_{n-2}, \mathcal{O}}$, and is isomorphic to the module $Y_{n-2, \mathcal{O}}^{\mathfrak{B}}$ (in the obvious notation). The operator $T_{s_{1}}$ preserves the subspace spanned by the vectors

$$
H_{s_{2} \ldots s_{n-3} s_{n-2} w_{0} s_{n-1}} \otimes 1, H_{s_{1} \ldots s_{n-3} s_{n-2} w_{0} s_{n-1}} \otimes 1,
$$

and the matrix of its restriction to this subspace is

$$
\left(\begin{array}{cc}
-\frac{q-1}{q^{m-2}-1} & 1 \\
\frac{q\left(q^{m-3}-1\right)\left(q^{m-1}-1\right)}{\left(q^{m-2}-1\right)^{2}} & \frac{q^{m-2}(q-1)}{q^{m-2}-1}
\end{array}\right) .
$$

It acts as multiplication by $q$ on the other basis vectors. It is now easy to see that the algebra $H_{\mathfrak{B}, \mathcal{O}}$ preserves $Y_{\mathcal{O}}^{\mathfrak{B}}$. The character of $\mathcal{O}[\Lambda]$ afforded by $X_{\mathcal{O}}^{\mathfrak{B}}$ is distinct from the other characters of $\mathcal{O}[\Lambda]$ appearing in $Y_{\mathcal{O}}^{\mathfrak{B}}$,

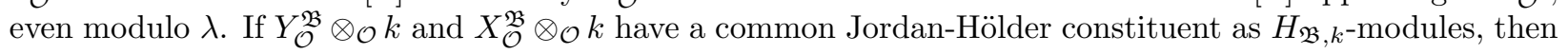
they must also have a common Jordan-Hölder constituent as $H_{\mathfrak{B}_{n-2}, k}$-modules, contradicting the induction hypothesis. This completes the proof of the proposition.

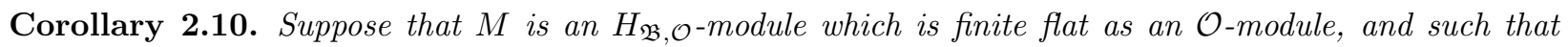
$M \otimes_{\mathcal{O}, \iota} \mathbb{C} \cong\left(X^{\mathfrak{B}}\right)^{a} \oplus\left(Y^{\mathfrak{B}}\right)^{b}$ for some integers $a, b \geq 0$. Suppose that $l \nmid q(q+1) \prod_{i=1}^{n / 2-2}\left(q^{i}-1\right)$. Let $M_{X}$ denote the intersection of $M$ with the $X_{K}^{\mathfrak{B}}$-isotypic component of $M \otimes_{\mathcal{O}} K$, and similarly for $M_{Y}$. Then there is a direct sum decomposition of $H_{\mathfrak{B}, \mathcal{O} \text {-modules }}$

$$
M=M_{X} \oplus M_{Y} .
$$


Proof. Consider the map $M_{X} \oplus M_{Y} \rightarrow M$. It is injective, and surjective after tensoring with $K$. To show that it is surjective, it suffices to show that the induced map

$$
M_{X} \otimes_{\mathcal{O}} k \oplus M_{Y} \otimes_{\mathcal{O}} k \rightarrow M \otimes_{\mathcal{O}} k
$$

is injective. However, the kernel of this map can be viewed as a submodule of $M_{X} \otimes_{\mathcal{O}} k$ and as a submodule

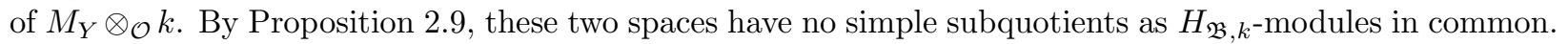
Therefore the kernel must be trivial, and this implies the result.

\section{Automorphic representations}

\section{$3.1 \mathrm{GL}_{n}$}

Let $p$ be a prime, and let $K$ be a finite extension of $\mathbb{Q}_{p}$. Let $\Omega$ denote an algebraically closed field of characteristic zero. There is a bijection

$$
\operatorname{rec}_{K}: \operatorname{Adm}_{\mathbb{C}} \operatorname{GL}_{n}(K) \leftrightarrow \mathrm{WD}_{\mathbb{C}}^{n} W_{K},
$$

characterized by a certain equality of epsilon- and L-factors on either side, cf. [HT01, Hen02. When $n=1$, it is induced by the local Artin map, normalized to take uniformizers to geometric Frobenius elements. Here we write $\operatorname{Adm}_{\Omega} \mathrm{GL}_{n}(K)$ for the set of isomorphism classes of irreducible admissible representations of this group over $\Omega$, and $\mathrm{WD}_{\Omega}^{n} W_{K}$ for the set of Frobenius-semisimple Weil-Deligne representations $(r, N)$ of $W_{K}$ valued in $\mathrm{GL}_{n}(\Omega)$. We define $\operatorname{rec}_{K}^{T}(\pi)=\operatorname{rec}_{K}\left(\pi|\cdot|^{(1-n) / 2}\right)$. This is the normalization of the local Langlands correspondence with good rationality properties; in particular, for any $\sigma \in \operatorname{Aut}(\mathbb{C})$ and any $\pi \in \operatorname{Adm}_{\mathbb{C}} \mathrm{GL}_{n}(K)$ there is an isomorphism

$$
\operatorname{rec}_{K}^{T}\left({ }^{\sigma} \pi\right) \cong{ }^{\sigma} \operatorname{rec}_{K}^{T}(\pi) .
$$

This can be seen using, for example, the characterization of $\operatorname{rec}_{K}$ and the description given in [Tat79, §3] of the action of Galois on local $\epsilon$ - and $L$-factors. It follows that for any $\Omega$ we can define a canonical bijection

$$
\operatorname{rec}_{K}^{T}: \operatorname{Adm}_{\Omega} \operatorname{GL}_{n}(K) \leftrightarrow \mathrm{WD}_{\Omega}^{n} W_{K} .
$$

Suppose instead that $K$ is a finite extension of $\mathbb{R}$. Then there is a bijection (Langlands' normalization):

$$
\operatorname{rec}_{K}: \operatorname{Adm}_{\mathbb{C}} \operatorname{GL}_{n}(K) \leftrightarrow \operatorname{Rep}_{\mathbb{C}}^{n} W_{K}
$$

Here we write $\operatorname{Adm}_{\mathbb{C}} \mathrm{GL}_{n}(K)$ for the set of infinitesimal equivalence classes of irreducible admissible representations of $\mathrm{GL}_{n}(K)$ and $\operatorname{Rep}_{\mathbb{C}}^{n} W_{K}$ for the set of continuous semisimple representations of $W_{K}$ into $\mathrm{GL}_{n}(\mathbb{C})$.

We define $\operatorname{rec}_{K}^{T}(\pi)=\operatorname{rec}_{K}\left(\pi|\cdot|^{(1-n) / 2}\right)$. If $K$ is isomorphic to $\mathbb{C}$, then we say that a character $K^{\times} \rightarrow \mathbb{C}^{\times}$is algebraic if it has the form $x \mapsto \sigma(x)^{n_{\sigma}} \tau(x)^{n_{\tau}}$ for some integers $n_{\sigma}, n_{\tau}$, and $\sigma, \tau$ the distinct $\mathbb{R}$-isomorphisms $K \cong \mathbb{C}$. We then say that $\pi$ is regular algebraic if $\operatorname{rec}_{K}^{T}(\pi)$ is a direct sum of pairwise distinct algebraic characters. If $K=\mathbb{R}$, we say that $\pi$ is regular algebraic if $\left.\operatorname{rec}_{K}^{T}(\pi)\right|_{\mathbb{C}^{\times}}$is a direct sum of pairwise distinct algebraic characters.

Now let $E$ be an imaginary $\mathrm{CM}$ field. We write $F$ for the maximal totally real subfield of $E$, and $c \in \operatorname{Gal}(E / F)$ for the non-trivial element.

Definition 3.1. 1. We say that an automorphic representation $\pi$ of $\mathrm{GL}_{n}\left(\mathbb{A}_{E}\right)$ is $R A C S D C$ (regular algebraic, conjugate self-dual, cuspidal) if it satisfies the following conditions:

- It is conjugate self-dual: $\pi^{c} \cong \pi^{\vee}$.

- It is cuspidal.

- It is regular algebraic.

2. We say that a pair $(\pi, \chi)$ of an automorphic representation $\pi$ of $\mathrm{GL}_{n}\left(\mathbb{A}_{E}\right)$ and a character $\chi$ : $F^{\times} \backslash \mathbb{A}_{F}^{\times} \rightarrow \mathbb{C}^{\times}$is RAECSDC (regular algebraic, essentially conjugate self-dual, cuspidal) if it satisfies the following conditions: 
- It is essentially conjugate self-dual: $\pi^{c} \cong \pi^{\vee} \otimes \chi \circ \mathbb{N}_{E / F}$.

- $\pi$ is cuspidal.

- $\pi$ is regular algebraic.

- $\chi$ is an algebraic character such that $\chi_{v}(-1)=(-1)^{n}$ for each place $v \mid \infty$.

3. We say that a pair $(\pi, \chi)$ of an automorphic representation $\pi$ of $\mathrm{GL}_{n}\left(\mathbb{A}_{F}\right)$ and a character $\chi$ : $F^{\times} \backslash \mathbb{A}_{F}^{\times} \rightarrow \mathbb{C}^{\times}$is RAESDC (regular algebraic, essentially self-dual, cuspidal) if it satisfies the following conditions:

- It is essentially self-dual: $\pi \cong \pi^{\vee} \otimes \chi$.

- $\pi$ is cuspidal.

- $\pi$ is regular algebraic.

- $\chi$ is an algebraic character such that $\chi_{v}(-1)$ is independent of the place $v \mid \infty$.

If $\pi$ is a regular algebraic cuspidal automorphic representation of $\mathrm{GL}_{n}\left(\mathbb{A}_{E}\right)$, then for each embedding $\tau: E \hookrightarrow \mathbb{C}$, we are given a representation $r_{\tau}: \mathbb{C}^{\times} \rightarrow \mathrm{GL}_{n}(\mathbb{C})$, induced by $\operatorname{rec}_{E_{v}}\left(\pi_{v}\right)$, where $v$ is the infinite place induced by $\tau$, and the isomorphism $E_{v}^{\times} \cong \mathbb{C}^{\times}$induced by $\tau$. There exists (cf. [Clo90b, Lemma 4.9]) an integer $w \in \mathbb{Z}$ such that this representation has the form

$$
r_{\tau}(z)=\left(z^{a_{\tau, 1}} \bar{z}^{w-a_{\tau, 1}}, \ldots, z^{a_{\tau, n}} \bar{z}^{w-a_{\tau, n}}\right),
$$

where $a_{\tau, i} \in(n-1) / 2+\mathbb{Z}$ and $a_{\tau, 1}>\cdots>a_{\tau, n}$. (Note that $w=0$ if and only if $\pi$ is unitary; this will be the case if $\pi$ is conjugate self-dual.) We will refer to the tuple $\mathbf{a}=\left(a_{\tau, 1}, \ldots, a_{\tau, n}\right)_{\tau \in \operatorname{Hom}(E, \mathbb{C})}$ as the infinity type of $\pi$. We also define a tuple $\boldsymbol{\lambda}=\left(\lambda_{\tau}\right)_{\tau \in \operatorname{Hom}(E, \mathbb{C})}=\left(\lambda_{\tau, 1}, \ldots, \lambda_{\tau, n}\right)_{\tau \in \operatorname{Hom}(E, \mathbb{C})}$, which we call the weight of $\pi$, by the formula $\lambda_{\tau, i}=-a_{\tau, n+1-i}+(n-1) / 2-(n-i)$. Then for each $\tau: E \hookrightarrow \mathbb{C}$, we have $\lambda_{\tau, 1} \geq \cdots \geq \lambda_{\tau, n}$, and the irreducible admissible representation of $\mathrm{GL}_{n}(\mathbb{C})$ corresponding to $r_{\tau}$ has the same infinitesimal character as the dual of the algebraic representation of $\mathrm{GL}_{n}(\mathbb{C})$ with highest weight $\lambda_{\tau}$. If $\pi$ is a regular algebraic cuspidal automorphic representation of $\mathrm{GL}_{n}\left(\mathbb{A}_{F}\right)$, then for each embedding $F \hookrightarrow \mathbb{C}$, we get a representation $r_{\tau}=\left.\operatorname{rec}_{F_{v}}\left(\pi_{v}\right)\right|_{\mathbb{C}^{\times}}$, where $v$ is the place of $F$ corresponding to $\tau$. In this case we use the same formulae to define the infinity type and the weight of $\pi$.

We will also have cause to consider representations which are not cuspidal. Suppose that $\sigma_{1}, \sigma_{2}$ are conjugate self-dual cuspidal automorphic representations of $\mathrm{GL}_{n_{1}}\left(\mathbb{A}_{E}\right), \mathrm{GL}_{n_{2}}\left(\mathbb{A}_{E}\right)$, respectively, and that $\Sigma=\sigma_{1} \boxplus \sigma_{2}$ is regular algebraic. Then the representations $\sigma_{i}|\cdot|{ }^{\left(n_{i}-n\right) / 2}$ are regular algebraic. We call a representation $\Sigma$ arising in this way a RACSD sum of cuspidal representations. In this case, define $\mathbf{a}^{i}=\left(a_{\tau}^{i}\right)_{\tau \in \operatorname{Hom}(E, \mathbb{C})}$ by the requirement that $\left(a_{\tau, 1}^{i}+\left(n_{i}-n\right) / 2, \ldots, a_{\tau, n_{i}}^{i}+\left(n_{i}-n\right) / 2\right)$ equal the infinity type of $\sigma_{i}|\cdot|^{\left(n_{i}-n\right) / 2}$, and define $\mathbf{b}=\left(b_{\tau}\right)_{\tau \in \operatorname{Hom}(E, \mathbb{C})}$ by the formula

$$
\left(b_{\tau, 1}, \ldots, b_{\tau, n}\right)=\left(a_{\tau, 1}^{1}, \ldots, a_{\tau, n_{1}}^{1}, a_{\tau, 1}^{2}, \ldots, a_{\tau, n_{2}}^{2}\right) .
$$

Let $\mathfrak{S}_{n}$ denote the symmetric group on $\{1, \ldots, n\}$. There is a unique tuple $\mathbf{w}=\left(w_{\tau}\right)_{\tau \in \operatorname{Hom}(E, \mathbb{C})} \in \mathfrak{S}_{n}^{\operatorname{Hom}(E, \mathbb{C})}$ such that for each $\tau \in \operatorname{Hom}(E, \mathbb{C})$, we have

$$
b_{\tau, w_{\tau}(1)}>\cdots>b_{\tau, w_{\tau}(n)} .
$$

The infinity type of $\Sigma$ is defined to be $\left(b_{\tau, w_{\tau}(1)}, \ldots, b_{\tau, w_{\tau}(n)}\right)_{\tau \in \operatorname{Hom}(E, \mathbb{C})}$.

Theorem 3.2. $\quad$ 1. Let $\pi$ be a RACSD sum of cuspidals or a RAECSDC automorphic representation of $\mathrm{GL}_{n}\left(\mathbb{A}_{E}\right)$, and fix an isomorphism $\iota: \overline{\mathbb{Q}}_{l} \cong \mathbb{C}$. Then there exists a continuous semisimple representation

$$
r_{\iota}(\pi): G_{E} \rightarrow \mathrm{GL}_{n}\left(\overline{\mathbb{Q}}_{l}\right)
$$

satisfying the following property: for every finite place $v$ of $E$ not dividing $l$, there is an isomorphism

$$
W D\left(\left.r_{\iota}(\pi)\right|_{G_{E v}}\right)^{F-s s} \cong \operatorname{rec}_{E_{v}}^{T}\left(\iota^{-1} \pi_{v}\right) .
$$

For each place $v$ of $E$ dividing $l,\left.r_{\iota}(\pi)\right|_{G_{E_{v}}}$ is de Rham, and if $\tau: E_{v} \hookrightarrow \overline{\mathbb{Q}}_{l}$ is an embedding and $\mathbf{a}$ the infinity type of $\pi$, then the Hodge-Tate weights with respect to this embedding are

$$
\operatorname{HT}_{\tau}\left(r_{\iota}(\pi)\right)=\left\{-a_{\iota^{-1} \tau, 1}+(n-1) / 2, \ldots,-a_{\iota^{-1} \tau, n}+(n-1) / 2\right\} .
$$


2. Let $(\pi, \chi)$ be a RAESDC automorphic representation of $\mathrm{GL}_{n}\left(\mathbb{A}_{F}\right)$, and fix an isomorphism $\iota: \overline{\mathbb{Q}}_{l} \cong \mathbb{C}$. Then there exists a continuous semisimple representation

$$
r_{\iota}(\pi): G_{F} \rightarrow \mathrm{GL}_{n}\left(\overline{\mathbb{Q}}_{l}\right)
$$

satisfying the following property: for every finite place $v$ of $F$ not dividing $l$, there is an isomorphism

$$
W D\left(\left.r_{\iota}(\pi)\right|_{G_{E_{v}}}\right)^{F-s s} \cong \operatorname{rec}_{F_{v}}^{T}\left(\iota^{-1} \pi_{v}\right)
$$

For each place $v$ of $F$ dividing $l,\left.r_{\iota}(\pi)\right|_{G_{F}}$ is de Rham, and if $\tau: F_{v} \hookrightarrow \overline{\mathbb{Q}}_{l}$ is an embedding and $\mathbf{a}$ the infinity type of $\pi$, then the Hodge-Tate weights with respect to this embedding are

$$
\operatorname{HT}_{\tau}\left(r_{\iota}(\pi)\right)=\left\{-a_{\iota^{-1} \tau, 1}+(n-1) / 2, \ldots,-a_{\iota^{-1} \tau, n}+(n-1) / 2\right\} .
$$

Proof. This theorem is due to many people. We give references for the case of a RACSDC automorphic representation $\pi$, from which the others can be deduced. In this case the existence of the representation $r_{\iota}(\pi)$ is proved in [CH, Theorem 3.2.3]. The strong form of local-global compatibility is proved in Car12.

Lemma 3.3. Let $\pi$ be one of the above types of automorphic representations, and fix an isomorphism $\iota: \overline{\mathbb{Q}}_{l} \cong \mathbb{C}$. Let $\sigma$ be a continuous automorphism of $\overline{\mathbb{Q}}_{l}$. Then ${ }^{\iota \sigma \iota^{-1}} \pi$ is defined, by [Clo90b, Theorem 3.13]. There are isomorphisms

$$
r_{\iota}\left({ }^{\iota \sigma \iota}{ }^{-1} \pi\right) \cong r_{\iota \sigma}(\pi) \cong \sigma r_{\iota}(\pi) .
$$

Proof. This follows from local-global compatibility, the rationality of the local Langlands correspondence for $\mathrm{GL}_{n}$, and the Chebotarev density theorem.

We will use the following convention for residual representations. If $\rho: G_{F} \rightarrow \mathrm{GL}_{n}\left(\overline{\mathbb{Q}}_{l}\right)$ is a continuous representation, then after choosing an invariant lattice, defined over a finite extension of $\mathbb{Q}_{l}$, we obtain by reduction modulo $l$ a residual representation valued in $\mathrm{GL}_{n}\left(\overline{\mathbb{F}}_{l}\right)$. By the principle of Brauer-Nesbitt, the semisimplification of this representation depends, up to isomorphism, only on $\rho$, and will be denoted $\bar{\rho}: G_{F} \rightarrow \mathrm{GL}_{n}\left(\overline{\mathbb{F}}_{l}\right)$.

\subsection{Ordinary forms}

Let $L=E$ or $F$. If $\pi$ is a regular algebraic automorphic representation of $\mathrm{GL}_{n}\left(\mathbb{A}_{L}\right)$ of infinity type a and weight $\boldsymbol{\lambda}$, we define Hecke operators $U_{\boldsymbol{\lambda}, v}^{j}$ as follows at primes $v$ above $l$. They depend on a choice of isomorphism $\iota: \overline{\mathbb{Q}}_{l} \cong \mathbb{C}$, which we fix for the rest of this section, as well as a choice of uniformizer $\varpi_{v}$ of $\mathcal{O}_{L_{v}}$. Define a matrix

$$
\alpha_{v}^{j}=\operatorname{diag}(\underbrace{\varpi_{v}, \ldots, \varpi_{v}}_{j}, \underbrace{1, \ldots, 1}_{n-j})
$$

and set

$$
U_{\boldsymbol{\lambda}, v}^{j}=\prod_{\tau} \iota^{-1} \tau\left(\varpi_{v}\right)^{-\lambda_{\tau, n}-\cdots-\lambda_{\tau, n+1-j}}\left[\operatorname{Iw}_{c}(v) \alpha_{v}^{j} \operatorname{Iw}_{c}(v)\right] .
$$

By definition, the subgroup $\operatorname{Iw}_{c}(v) \subset \operatorname{GL}_{n}\left(\mathcal{O}_{L_{v}}\right)$ is the subgroup of matrices whose reduction modulo $\varpi_{v}^{c}$ is an upper-triangular matrix with 1's on the diagonal, and the product runs over embeddings $\tau: L \hookrightarrow \mathbb{C}$ such that $\iota^{-1} \tau$ induces the place $v$ of $L$. We note that by [Ger, Lemma 2.3.3], the Hecke operators $U_{\boldsymbol{\lambda}, v}^{j}$ commute with the inclusions $\iota^{-1} \pi_{v}^{\mathrm{Iw}_{c}(v)} \rightarrow \iota^{-1} \pi_{v}^{\mathrm{Iw}_{c^{\prime}}(v)}$ when $c^{\prime} \geq c$. It therefore makes sense to omit $c$ from the notation defining $U_{\boldsymbol{\lambda}, v}^{j}$. We also write $T_{c}(v) \subset \operatorname{Iw}_{c}(v)$ for the group of diagonal matrices with integral entries which are congruent to 1 modulo $\varpi_{v}^{c}, e_{v}$ for the absolute ramification index of $\left[L_{v}: \mathbb{Q}_{l}\right], f_{v}$ for the absolute residue degree, and val $: \overline{\mathbb{Q}}_{l}^{\times} \rightarrow \mathbb{Q}$ for the valuation such that $\operatorname{val}(l)=1$.

Definition 3.4. Let $\pi$ be a regular algebraic automorphic representation of $\mathrm{GL}_{n}\left(\mathbb{A}_{L}\right)$ of weight $\boldsymbol{\lambda}$. We say that $\pi$ is $\iota$-ordinary if for each place $v$ of $L$ dividing $l$, there is an integer $c \geq 1$ and a line inside $\iota^{-1} \pi_{v}^{\operatorname{Iw}_{c}(v)}$ which is invariant under each operator $U_{\boldsymbol{\lambda}, v}^{j}$, and such that the eigenvalues of these operators on this line are all l-adic units. 
The next lemma follows immediately from [CT, Lemma 2.5] and [CT, Lemma 2.6].

Lemma 3.5. 1. The subspace of $\underset{c}{\lim } \iota^{-1} \pi_{v}^{\mathrm{Iw}_{c}(v)}$ where each operator $U_{\boldsymbol{\lambda}, v}^{j}$ acts with eigenvalue a unit has dimension at most one.

2. Suppose that $\pi_{1}, \pi_{2}$ are cuspidal conjugate self-dual automorphic representations of $\mathrm{GL}_{n_{1}}\left(\mathbb{A}_{E}\right)$ and $\mathrm{GL}_{n_{2}}\left(\mathbb{A}_{E}\right)$, respectively. Suppose that $\Pi=\pi_{1} \boxplus \pi_{2}$ is regular algebraic. Then the representations $\pi_{i}|\cdot|^{\left(n_{i}-n\right) / 2}$ are regular algebraic, and $\Pi$ is $\iota$-ordinary if and only if $\pi_{1}|\cdot|^{\left(n_{1}-n\right) / 2}, \pi_{2}|\cdot|^{\left(n_{2}-n\right) / 2}$ are $\iota$-ordinary and the following condition on infinity types holds. Recall the tuple $\mathbf{w}=\left(w_{\tau}\right)_{\tau \in \operatorname{Hom}(E, \mathbb{C})}$ of permutations associated to the infinity types of $\pi_{1}, \pi_{2}$. Then $w_{\tau}$ depends only on the place $v$ of $E$ dividing $l$ induced by the embedding $\iota^{-1} \tau: E \hookrightarrow \overline{\mathbb{Q}}_{l}$.

\subsection{Definite unitary groups}

We now let $E$ be an imaginary $\mathrm{CM}$ field with totally real subfield $F$, and suppose that $[F: \mathbb{Q}]$ is even. Let $G$ be a unitary group in $n$ variables associated to the extension $E / F$, quasi-split at every finite place, such that $G(\mathbb{R})$ is compact. Such a group exists since $[F: \mathbb{Q}]$ is even, and is uniquely determined up to isomorphism. We can choose the matrix algebra $B=M_{n}(E)$ and an involution $\dagger$ of $B$ of the second kind, so that $G$ is defined by

$$
G(R)=\left\{g \in\left(B \otimes_{F} R\right)^{\times} \mid g^{\dagger} g=1\right\}
$$

for any $F$-algebra $R$. We may choose an order $\mathcal{O}_{B} \subset B$, stable under $\dagger$, so that $\mathcal{O}_{B, w}$ is maximal for any place $w$ of $E$ split over $F$. This defines an integral model of $G$ over $\mathcal{O}_{F}$, and for any place $v$ of $F$ split as $v=w w^{c}$ in $E$, we can choose an isomorphism

$$
\mathcal{O}_{B} \otimes_{\mathcal{O}_{F}} \mathcal{O}_{F_{v}} \cong M_{n}\left(\mathcal{O}_{E_{w}}\right) \times M_{n}\left(\mathcal{O}_{E_{w^{c}}}\right),
$$

such that $\dagger$ acts as $\left(g_{1}, g_{2}\right) \mapsto\left(g_{2},{ }^{t} g_{1}\right)$. Projection onto the first factor induces an isomorphism $\iota_{w}: G\left(F_{v}\right) \rightarrow$ $\mathrm{GL}_{n}\left(E_{w}\right)$ such that $\iota_{w}\left(G\left(\mathcal{O}_{F_{v}}\right)\right)=\mathrm{GL}_{n}\left(\mathcal{O}_{E_{w}}\right)$.

Let $l$ be a prime, and suppose that every prime of $F$ above $l$ splits in $E$. Let $S_{l}$ denote the set of primes of $F$ above $l$. We choose a prime $\widetilde{v}$ of $E$ above $v$ for each $v \in S_{l}$, and let $\widetilde{S}_{l}$ denote the set of these primes. Then, as above, we are given an isomorphism $\iota_{\widetilde{v}}: G\left(F_{v}\right) \rightarrow \mathrm{GL}_{n}\left(E_{\widetilde{v}}\right)$. We write $I_{l}$ for the set of embeddings $F \hookrightarrow \overline{\mathbb{Q}}_{l}$, and $\widetilde{I}_{l}$ for the set of embeddings $E \hookrightarrow \overline{\mathbb{Q}}_{l}$ inducing an element of $\widetilde{S}_{l}$. These two sets are therefore in canonical bijection.

Let $K \subset \overline{\mathbb{Q}}_{l}$ be a finite extension of $\mathbb{Q}_{l}$, with ring of integers $\mathcal{O}$ and residue field $k$. We suppose that $K$ contains the image of $E$ under every embedding $E \hookrightarrow \overline{\mathbb{Q}}_{l}$. To a tuple $\boldsymbol{\lambda}=\left(\lambda_{\tau, 1}, \ldots, \lambda_{\tau, n}\right)_{\tau \in \widetilde{I}_{l}}$ of dominant weights of $\mathrm{GL}_{n}$, we associate a representation $M_{\boldsymbol{\lambda}}$ of the group $\prod_{v \in S_{l}} G\left(\mathcal{O}_{F_{v}}\right)$ as in Ger, Definition 2.2.3]. It is an $\mathcal{O}$-lattice inside the representation $W_{\boldsymbol{\lambda}}=\otimes_{\tau \in \widetilde{I}_{l}}\left(W_{\lambda_{\tau}} \otimes_{F_{v}, \tau} K\right)$, where $W_{\lambda_{\tau}}$ is the algebraic representation of $\mathrm{GL}_{n}\left(F_{v}\right)$ of highest weight $\lambda_{\tau}$, and $v$ is the place of $F$ induced by $\tau$.

Fix $\boldsymbol{\lambda}$ and an open compact subgroup $U=\prod_{v} U_{v} \subset G\left(\mathbb{A}_{F}^{\infty}\right)$, such that $U_{v} \subset G\left(\mathcal{O}_{F_{v}}\right)$ for each $v \in S_{l}$. Let $A$ be an $\mathcal{O}$-algebra. We can then define a space of automorphic forms with $A$-coefficients as follows. By definition, $S_{\boldsymbol{\lambda}}(U, A)$ is the set of functions $f: G(F) \backslash G\left(\mathbb{A}_{F}^{\infty}\right) \rightarrow M_{\boldsymbol{\lambda}} \otimes_{\mathcal{O}} A$ such that for all $u \in U$, we have $f(g u)=u_{l}^{-1} \cdot f(g)$. Here $u_{l}$ denotes the projection of $u$ to its $\prod_{v \in S_{l}} G\left(\mathcal{O}_{F_{v}}\right)$-component. If $\boldsymbol{\lambda}=0$, then we write $S_{\boldsymbol{\lambda}}(U, A)=S(U, A)$.

The relation with classical automorphic forms is given by the following result. Let $\mathcal{A}$ denote the space of automorphic forms on $G(F) \backslash G(\mathbb{A})$, and let $\iota: \overline{\mathbb{Q}}_{l} \cong \mathbb{C}$ be an isomorphism. There is an algebraic representation $W_{\iota \boldsymbol{\lambda}}$ of $G\left(F \otimes_{\mathbb{Q}} \mathbb{R}\right)$, defined by the formula $\otimes_{\tau \in \widetilde{I}_{l}} W_{\lambda_{\tau}} \otimes_{F_{v}, \iota \tau} \mathbb{C}$.

Proposition 3.6. There is a canonical isomorphism

$$
\left(\underset{\vec{U}}{\lim } S_{\boldsymbol{\lambda}}(U, K)\right) \otimes_{K, \iota} \mathbb{C} \cong \operatorname{Hom}_{G\left(F \otimes_{\mathbb{Q}} \mathbb{R}\right)}\left(W_{\iota \boldsymbol{\lambda}}^{\vee}, \mathcal{A}\right) .
$$

In particular, for any irreducible subrepresentation $\sigma \subset \mathcal{A}$, there is a canonical subspace $\iota^{-1}\left(\sigma^{\infty}\right)^{U} \subset$ $S_{\boldsymbol{\lambda}}\left(U, \overline{\mathbb{Q}}_{l}\right)$, and $\underset{U}{\lim } S_{\boldsymbol{\lambda}}(U, K)$ is a semisimple admissible representation of $G\left(\mathbb{A}_{F}^{\infty}\right)$. 
Proof. This is proved just as CHT08, Proposition 3.3.2].

If $\pi$ is an automorphic representation of $\mathrm{GL}_{n}\left(\mathbb{A}_{E}\right)$ and $\sigma$ is an automorphic representation of $G\left(\mathbb{A}_{F}\right)$, we say that $\pi$ is the base change of $\sigma$ if for any finite place $w$ of $E$, the following condition is satisfied:

- If $w$ is split over the place $v$ of $F$, then $\pi_{w}$ is the standard base change of $\sigma_{v}$.

- If $w$ is inert over the place $v$ of $F$ and $\sigma_{v}$ is unramified, then $\pi_{w}$ is the standard unramified base change of $\sigma_{v}$ (cf. [Mín11, Theorem 4.1]).

Proposition 3.7. $\quad$ 1. Suppose that $\sigma$ is an automorphic representation of $G\left(\mathbb{A}_{F}\right)$. Then there exist discrete and conjugate self-dual representations $\pi_{1}, \ldots, \pi_{s}$ of $\mathrm{GL}_{n}\left(\mathbb{A}_{E}\right)$ such that $\pi=\pi_{1} \boxplus \cdots \boxplus \pi_{s}$ is the base change of $\sigma$ in the above sense.

Proof. This follows from [Lab11a, Corollaire 5.3].

Proposition 3.8. Let $\sigma$ be an automorphic representation of $G\left(\mathbb{A}_{F}\right)$. Then there exists a unique continuous semisimple representation

$$
r_{\iota}(\sigma): G_{E} \rightarrow \mathrm{GL}_{n}\left(\overline{\mathbb{Q}}_{l}\right)
$$

satisfying the following condition: for every place $w$ of $E$ split over $F$, we have

$$
W D\left(\left.r_{\iota}(\sigma)\right|_{G_{E_{w}}}\right)^{F-s s} \cong \operatorname{rec}_{E_{w}}^{T}\left(\iota^{-1}\left(\sigma_{v} \circ \iota_{w}\right)\right) .
$$

Proof. This now follows from Theorem 3.2 .

Let $U=\prod_{v} U_{v}$ be an open compact subgroup as above, and suppose that there exists an integer $c \geq 1$ such that for each $v \in S_{l}, U_{v}=\iota_{\widetilde{v}}^{-1} \operatorname{Iw}_{c}(\widetilde{v})$. For each prime $v \in S_{l}$, fix a uniformizer $\varpi_{\widetilde{v}}$ of $\mathcal{O}_{E_{\widetilde{v}}}$, and define the matrix

$$
\alpha_{v}^{j}=\operatorname{diag}(\underbrace{\varpi_{\widetilde{v}}, \ldots, \varpi_{\widetilde{v}}}_{j}, \underbrace{1, \ldots, 1}_{n-j}) .
$$

We define an endomorphism $U_{\boldsymbol{\lambda}, v}^{j}$ of the space $S_{\boldsymbol{\lambda}}(U, \mathcal{O})$ by the formula

$$
U_{\boldsymbol{\lambda}, v}^{j}=\prod_{\tau} \iota^{-1} \tau\left(\varpi_{\widetilde{v}}\right)^{-\lambda_{\tau, n}-\cdots-\lambda_{\tau, n+1-j}} \iota_{\widetilde{v}}^{-1}\left[\operatorname{Iw}_{c}(\widetilde{v}) \alpha_{v}^{j} \operatorname{Iw}_{c}(\widetilde{v})\right] .
$$

If $\boldsymbol{\lambda}=0$, then we write $U_{\boldsymbol{\lambda}, v}^{j}=U_{v}^{j}$. If $\sigma$ is an automorphic representation of $G\left(\mathbb{A}_{F}\right)$, we say that $\sigma$ is $\iota$-ordinary if there exists an integer $c \geq 1$ and an open compact subgroup $U$ of this form such that these operators on $\left(\iota^{-1} \sigma^{\infty}\right)^{U}$ have a common line where they all act with eigenvalues which are $l$-adic units.

Lemma 3.9. 1. Let $\sigma$ be an automorphic representation of $G\left(\mathbb{A}_{F}\right)$, and let $\pi$ denote its base change to

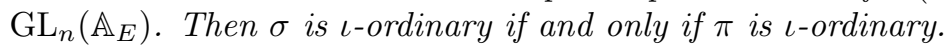

2. Let $v \in S_{l}$. Then the subspace $\iota^{-1} \sigma_{v}^{\text {ord }}$ of $\underset{c}{\lim _{c} \iota^{-1} \sigma_{v}^{\iota \tilde{v}^{-1}} \operatorname{Iw}_{c}(\widetilde{v})}$ where each operator $U_{\boldsymbol{\lambda}, v}^{j}$ acts with eigenvalues which are l-adic units has dimension at most one. If $\sigma_{v}^{\tau_{v}^{-1} \operatorname{Iw}_{c}(\widetilde{v})} \neq 0$ then we have $\iota^{-1} \sigma_{v}^{\text {ord }} \subset \iota^{-1} \sigma_{v}^{\iota \widetilde{v}^{-1} \operatorname{Iw}_{c}(\widetilde{v})}$.

Proof. Since $l$ is split, by assumption, this follows from the corresponding facts for $\mathrm{GL}_{n}\left(\mathbb{A}_{E}\right)$ and the definition of base change.

Let $\sigma$ be an automorphic representation of $G\left(\mathbb{A}_{F}\right)$. We will write $\left(\iota^{-1} \sigma^{\infty}\right)^{\text {ord }}$ for the subspace

$$
\iota^{-1} \sigma^{l, \infty} \otimes \bigotimes_{v \in S_{l}} \iota^{-1} \sigma_{v}^{\text {ord }} \subset \iota^{-1} \sigma^{\infty}
$$

This is an admissible representation of $G\left(\mathbb{A}_{F}^{l, \infty}\right)$, and is non-zero precisely when $\sigma$ is $\iota$-ordinary. 
Proposition 3.10. Let $\sigma$ be an ı-ordinary automorphic representation of $G\left(\mathbb{A}_{F}\right)$ of weight $\boldsymbol{\lambda}$. Let $U=$ $\prod_{v} U_{v} \subset G\left(\mathbb{A}_{F}^{\infty}\right)$ be an open compact subgroup as above, and suppose that $\sigma^{U} \neq 0$. Let $\boldsymbol{\lambda}^{\prime}$ be another choice of weight. Then there exists an ı-ordinary automorphic representation $\sigma^{\prime}$ of $G\left(\mathbb{A}_{F}\right)$ of weight $\boldsymbol{\lambda}^{\prime}$ such that $\overline{r_{\iota}(\sigma)} \cong \overline{r_{\iota}\left(\sigma^{\prime}\right)}$ and for every finite place $v$ of $F$ not dividing $l,\left(\sigma_{v}^{\prime}\right)^{U_{v}} \neq 0$.

Proof. This is an easy consequence of Hida theory, cf. [Ger, Lemma 2.6.4]. In this reference it is assumed that the extension $E / F$ is everywhere unramified, but in our situation this makes no difference.

\subsection{Endoscopic transfer}

We continue with the notation and assumptions of the previous section. We make the following further hypotheses:

$-n \geq 6$ is even.

- There exist places $v_{0}, \ldots, v_{s}$ of $F$ not dividing $2 l$ which are ramified in $E$. We write $w_{0}, \ldots, w_{s}$ for the places of $E$ above $v_{0}, \ldots, v_{s}$.

- $E / F$ is unramified at every finite place $v \neq v_{0}, \ldots, v_{s}$.

- For each place $v$ of $F$ dividing $l$, the local degree $\left[F_{v}: \mathbb{Q}_{l}\right]$ is even.

We fix for each place $v$ of $F$ inert in $E$ a hyperspecial maximal compact subgroup $U_{v} \subset G\left(F_{v}\right)$. We fix also an isomorphism $\iota: \overline{\mathbb{Q}}_{l} \cong \mathbb{C}$. For each $i=0, \ldots, s$ we have defined an L-packet $\left\{X_{i}, Y_{i}\right\}$ of representations of $G\left(F_{v_{i}}\right)$, cf. Theorem 2.7. Define a function $\epsilon_{i}:\left\{X_{i}, Y_{i}\right\} \rightarrow\{ \pm 1\}$, by

$$
\epsilon_{i}\left(X_{i}\right)=-1, \epsilon_{i}\left(Y_{i}\right)=+1 .
$$

Theorem 3.11. 1. Let $\pi_{1}, \pi_{2}$ be RACSDC automorphic representations of $\mathrm{GL}_{2}\left(\mathbb{A}_{E}\right), \mathrm{GL}_{n-2}\left(\mathbb{A}_{E}\right)$, respectively. Suppose that $\pi=\pi_{1} \boxplus \pi_{2}$ satisfies the following:

$-\pi$ has weight zero.

- $\pi$ is ı-ordinary.

- If $w \neq w_{0}, \ldots, w_{s}$ is a place of $E$ at which $\pi_{w}$ is ramified, then $w$ is split over $F$.

- For each $i=0, \ldots, s$ we have $\pi_{1, w_{i}} \cong \mathrm{St}_{2, E_{w_{i}}}$ and $\pi_{2, w_{i}} \cong \mathrm{St}_{n-2, E_{w_{i}}}$.

Then there are exactly $2^{s}$ automorphic representations $\sigma$ of $G\left(\mathbb{A}_{F}\right)$ which have base change equal to $\pi$, and such that if $v$ is a place of $F$ inert in $E$, then $\sigma_{v}^{U_{v}} \neq 0$. They are in bijective correspondence with elements $\mathbf{d} \in \prod_{i=1}^{s}\left\{X_{i}, Y_{i}\right\}$, this correspondence $\mathbf{d} \leftrightarrow \sigma(\mathbf{d})$ being characterized by the relation

$$
\sigma(\mathbf{d})_{v_{i}} \cong d_{i}, i=1, \ldots, s .
$$

These representations each appear with multiplicity one, and satisfy the further condition

$$
\epsilon_{0}\left(\sigma(\mathbf{d})_{v_{0}}\right) \cdot \prod_{i=1}^{s} \epsilon_{i}\left(d_{i}\right)=1
$$

2. Suppose that $\pi$ is a RACSDC automorphic representation of $\mathrm{GL}_{n}\left(\mathbb{A}_{E}\right)$ satisfying the following:

- If $w \neq w_{0}, \ldots, w_{s}$ is a place of $E$ at which $\pi_{w}$ is ramified, then $w$ is split over $F$.

- $\pi$ has weight zero.

- For each $i=0, \ldots, s, \pi_{w_{i}} \cong \mathrm{St}_{2, E_{w_{i}}} \boxplus \mathrm{St}_{n-2, E_{w_{i}}}$.

Then there are exactly $2^{s+1}$ automorphic representations $\sigma$ of $G\left(\mathbb{A}_{F}\right)$ such that $\pi$ is the base change of $\sigma$ and such that if $v$ is a place of $F$ inert in $E$, then $\sigma_{v}^{U_{v}} \neq 0$. They are in bijective correspondence with elements $\mathbf{d} \in \prod_{i=0}^{s}\left\{X_{i}, Y_{i}\right\}$, this correspondence $\mathbf{d} \leftrightarrow \sigma(\mathbf{d})$ being characterized by the relation

$$
\sigma(\mathbf{d})_{v_{i}} \cong d_{i}, i=0, \ldots, s .
$$

These representations each appear with multiplicity one.

3. Suppose that $\pi$ is a RACSDC automorphic representation of $\mathrm{GL}_{n}\left(\mathbb{A}_{E}\right)$ satisfying the following:

- If $w \neq w_{0}, \ldots, w_{s}$ is a place of $E$ at which $\pi_{w}$ is ramified, then $w$ is split over $F$.

$-\pi$ has weight zero. 
- $\pi_{w_{0}}$ is an unramified twist of the Steinberg representation.

- For each $i=1, \ldots, s, \pi_{w_{i}} \cong \mathrm{St}_{2, E_{w_{i}}} \boxplus \mathrm{St}_{n-2, E_{w_{i}}}$.

Then there are exactly $2^{s}$ automorphic representations $\sigma$ of $G\left(\mathbb{A}_{F}\right)$ such that $\pi$ is the base change of $\sigma$ and such that if $v$ is a place of $F$ inert in $E$, then $\sigma_{v}^{U_{v}} \neq 0$. They are in bijective correspondence with elements $\mathbf{d} \in \prod_{i=1}^{s}\left\{X_{i}, Y_{i}\right\}$, this correspondence $\mathbf{d} \leftrightarrow \sigma(\mathbf{d})$ being characterized by the relation

$$
\sigma(\mathbf{d})_{v_{i}} \cong d_{i}, i=1, \ldots, s .
$$

These representations each appear with multiplicity one.

The rest of $₫ 3$ will be devoted to the proof of Theorem 3.11, which depends on the stabilization of the trace formula for the definite unitary group $G$. We write $G^{*}$ for the quasi-split inner form of $G$. The other elliptic endoscopic groups of $G$ are isomorphic to $U(a) \times U(b), a, b \geq 1, a+b=n$, where $U(m)$ denotes the quasi-split unitary group in $m$ variables attached to the extension $E / F$. We will be especially interested in the group

$$
H=U(2) \times U(n-2) .
$$

We recall that we have defined an L-embedding $\xi:{ }^{L} H \rightarrow{ }^{L} G$ as

$$
\begin{gathered}
\xi\left(g_{1}, g_{2}, w\right)=\left(\left(\begin{array}{cc}
g_{1} & 0 \\
0 & g_{2}
\end{array}\right), w\right)\left(w \in W_{E}\right), \\
\xi\left(w_{c}\right)=\left(\left(\begin{array}{cc}
\Phi_{2} & 0 \\
0 & \Phi_{n-2}
\end{array}\right) \Phi_{n}^{-1}, w_{c}\right)
\end{gathered}
$$

where $w_{c} \in W_{F}$ is a representative of complex conjugation. Stable base change is associated to the L-group homomorphism

$$
\begin{gathered}
{ }^{L} G={ }^{L} G^{*} \rightarrow{ }^{L}\left(\operatorname{Res}_{E / F} G\right)=\mathrm{GL}_{n}(\mathbb{C}) \times \mathrm{GL}_{n}(\mathbb{C}) \rtimes W_{F}, \\
(g, w) \mapsto(g, g, w)
\end{gathered}
$$

(cf. Mín11, p. 402], Mok, (2.1.9)]). The analogue of the above theorem with $G$ replaced by its quasisplit inner form $G^{*}$ has been proved by Mok [Mok, Theorem 2.5.2]. Let $\pi$ be one of the automorphic representations of $\mathrm{GL}_{n}\left(\mathbb{A}_{E}\right)$ appearing in the statement of the theorem, and let $S$ be a finite set of places of $F$ containing the archimedean primes and the places below which $\pi$ is ramified. Let $\psi$ denote the data of the Hecke matrix $t_{\pi_{w}}$ for $w$ coprime to $S$ (this is the data used by Arthur [Art13] and Mok). By unramified base change [Mín11], $\psi$ defines an unramified representation $\sigma_{v}$ of $G^{*}\left(F_{v}\right)$ for $v \notin S$, characterized for $v$ inert in $E$ by the property $\sigma_{v}^{U_{v}} \neq 0$.

Mok describes the full subspace of $L_{\text {disc }}^{2}\left(G^{*}(F) \backslash G^{*}\left(\mathbb{A}_{F}\right)\right)$ associated to $\psi$. At the archimedean places and the places $v_{0}, \ldots, v_{s}$, there is an L-packet $\Pi\left(\psi_{v}\right)$ of representations of $G^{*}\left(F_{v}\right)$ and the choice of a local representation $\sigma_{v} \in \Pi\left(\psi_{v}\right)$ is subject to a global sign condition, cf. [Mok, Theorem 2.5.2]. If $\pi$ is cuspidal, then this condition is vacuous and every representation $\sigma$ in the global L-packet (restricted direct product of local L-packets) appears with multiplicity one.

We need the analogous result, however, for $G$ and not $G^{*}$, and Arthur's description of the spectrum has not been achieved in this case. We will deduce what we need from Mok's results; we apologise for the obvious redundancy of our arguments.

\subsection{Geometric transfer factors}

Assume $f=\otimes_{v} f_{v}$ is a decomposed, smooth, $K_{\infty}$-finite function on $G\left(\mathbb{A}_{F}\right)$. We will need the associated functions $f^{H}$ on $H\left(\mathbb{A}_{F}\right)$, where $H$ is an endoscopic group for $G$ (or $G^{*}$ ). This depends on a choice of transfer factors $\Delta(\gamma, \delta)$, where $(\gamma, \delta)$ are associated (strongly regular) elements in $H\left(F_{v}\right), G\left(F_{v}\right)$.

At the finite places, we use the Whittaker normalization of transfer factors [KS99, §5.3], [Mok, §3]. This is possible since $G$ is quasi-split at the finite places. At the archimedean places we will use Kottwitz's transfer factors, explicitly described for unitary groups in [Clo11]. We must check that such choices are 
compatible, i.e. that they satisfy a product formula for rational elements $(\gamma, \delta)$. The local factors at the finite places are defined by the formula

$$
\Delta(\gamma, \delta)=\epsilon(V, \psi) \Delta_{0}(\gamma, \delta),
$$

where $\Delta_{0}$ is the Langlands-Shelstad factor in the quasi-split case [KS99, p. 65]. Here $V=V_{G}-V_{H}$ is a virtual representation of $\operatorname{Gal}\left(\bar{F}_{v} / F_{v}\right)$, with $V_{G}=X_{*}\left(\widehat{T}_{G}\right) \otimes \mathbb{C}$, and similarly for $V_{H}$, and $\psi$ is an additive character. In our case, $V_{G} \cong V_{H}$ and $\Delta(\gamma, \delta)=\Delta_{0}(\gamma, \delta)$.

For $\gamma, \delta \in H\left(F_{\infty}\right), G\left(F_{\infty}\right)$, consider now the product $\Delta_{K, \infty}(\gamma, \delta)$ of Kottwitz's transfer factors at the real primes. If $G$ is replaced by $G^{*}$, then

$$
\Delta_{K, \infty}^{G^{*}}(\gamma, \delta)=\left( \pm(i)^{2(n-2)}\right)^{d} \Delta_{0, \infty}(\gamma, \delta)
$$

([Lab11b p. 414]), where $i=\sqrt{-1}$ and $d=[F: \mathbb{Q}]$. We now use Labesse's argument: the groups $G, G^{*}$, and $H$ can be chosen so as to contain the diagonal torus $T=U(1)^{n}$ (compatibly with [Clo11]). For $\gamma \in T$,

$$
\Delta_{K, \infty}^{G^{*}}(\gamma, \gamma)=\prod_{v \mid \infty}(-1)^{q\left(G^{*}\right)-q(G)} \Delta_{K, \infty}^{G}(\gamma, \gamma)
$$

where $q(G)$ is half the real dimension of the symmetric space of $G$ [Lab11b, p. 414]. In our case, then,

$$
\Delta_{K, \infty}^{G}(\gamma, \gamma)=\epsilon^{d} \Delta_{0, \infty}(\gamma, \gamma)
$$

on $T$, where $\epsilon= \pm i^{2(n-2)}(-1)^{n / 2}$. The two factors therefore coincide on $T$. The compatibility of the factors $\Delta_{0}$ thus implies that of our chosen factors on $T$ and therefore on $(G, H)$ by the essential uniqueness of local transfer factors.

\subsection{Spectral transfer factors, real places}

Once we have defined the association of $f$ and $f^{H}$, there follow identities between (signed) traces of $f$ and $f^{H}$ in associated L-packets. We describe this in the case of interest to us, namely when the global parameter $\psi$ arises from a regular algebraic automorphic representation $\pi=\pi_{1} \boxplus \pi_{2}$ of weight zero, and $\pi_{1}$, $\pi_{2}$ are RACSDC. Note that the datum of $\psi$, outside an arbitrary finite set $S$, uniquely determines $\pi_{1}$ and $\pi_{2}$, thus their infinity types, by the theorems of Jacquet-Shalika. In particular, it makes sense to consider the induced local parameter $\psi_{v}$ at an infinite place $v$ of $E$, cf. [Mok, $\S 2.3$ ].

Let us write $\mathbf{a}, \mathbf{b}$ for the infinity types of $\pi_{1}, \pi_{2}$, respectively, cf. 3.1. We recall that we have defined a tuple of permutations $\mathbf{w}=\left(w_{\tau}\right)_{\tau: E \hookrightarrow \mathbb{C}}$ in terms of these infinity types. The Langlands parameters of $\pi_{1}$, $\pi_{2}$ at the infinite place induced by an embedding $\tau: E \hookrightarrow \mathbb{C}$ are given by homomorphisms

$$
\begin{gathered}
z \mapsto\left((z / \bar{z})^{a_{\tau, 1}},(z / \bar{z})^{a_{\tau, 2}}\right), \\
z \mapsto\left((z / \bar{z})^{b_{\tau, 1}},(z / \bar{z})^{b_{\tau, 2}}, \ldots,(z / \bar{z})^{b_{\tau, n-2}}\right) .
\end{gathered}
$$

Let $\psi_{v, H}: W_{\mathbb{R}} \rightarrow{ }^{L} H$ be the sum of our two parameters, uniquely extended to $W_{\mathbb{R}}$. Let $\sigma_{v}$ denote the trivial representation of $G\left(F_{v}\right)$ for $v \mid \infty$, associated to the parameter

$$
\psi_{v}: z \mapsto\left((z / \bar{z})^{(n-1) / 2}, \ldots,(z / \bar{z})^{(1-n) / 2}\right)
$$

(extended to $\left.W_{\mathbb{R}}\right)$. There is a spectral transfer factor $\Delta_{v}\left(\psi_{v, H}, \sigma_{v}\right)$ satisfying the identity, for $f_{v}, f_{v}^{H}$ associated:

$$
\left\langle\Theta_{\psi_{v}, H}, f_{v}^{H}\right\rangle=\Delta_{v}\left(\psi_{v, H}, \sigma_{v}\right)\left\langle\Theta_{\sigma_{v}}, f_{v}\right\rangle .
$$

In this identity $\Theta_{\psi_{v, H}}$ is the stable character on $H\left(F_{v}\right)$ associated to the L-packet given by $\psi_{v, H}$; $\Theta_{\sigma_{v}}$ is the character of the trivial representation. (We note once and for all that in the identity

$$
S O_{\delta}\left(f^{H}\right)=\sum_{\gamma} \Delta(\gamma, \delta) O_{\gamma}(f)
$$

there is an implicit choice of Haar measures on $H\left(F_{v}\right)$ and $G\left(F_{v}\right)$. The same measures are used to define the integrals against $\Theta_{\psi_{v, H}}$ and $\Theta_{\sigma_{v}}$.) 
Lemma 3.12. For any $v \mid \infty, \Delta\left(\psi_{v, H}, \sigma_{v}\right)=\operatorname{det} w_{\tau}$, where $\tau: E \hookrightarrow \mathbb{C}$ is an embedding inducing the place $v$ of $F$.

Proof. This follows immediately from the exposition in [Clo11].

\subsection{Spectral transfer factors, $p$-adic places}

We now describe the character identities at the $p$-adic primes $v_{i}$, for the particular representations which will concern us. We first recall the characterization of the $L$-packet $\left\{X_{i}, Y_{i}\right\}$ associated to the representation $\Pi_{E}=\mathrm{St}_{2, E_{w_{i}}} \boxplus \mathrm{St}_{n-2, E_{w_{i}}}$. Recall the correspondence of $\$ 2.2$ between functions $f \in C_{c}^{\infty}\left(G^{*}\left(F_{v_{i}}\right)\right)$ and $f_{E} \in C_{c}^{\infty}\left(\mathrm{GL}_{n}\left(E_{w_{i}}\right)\right)$, characterized by an identity of stable (twisted) orbital integrals. Then for $f, f_{E}$ associated,

$$
\left\langle\operatorname{tr} X_{i}+\operatorname{tr} Y_{i}, f\right\rangle=\left\langle\Pi_{E} \times I_{c}, f_{E}\right\rangle
$$

where $I_{c}$ is the intertwining operator $\Pi_{E} \cong \Pi_{E}^{c}$, normalized by the Whittaker model, cf. [Mok, Theorem 3.2.1]. Consider now the endoscopic group $H=U(2) \times U(n-2)$. The parameter $\psi_{v_{i}}$ can be seen as a parameter $\psi_{v_{i}, H}$ for $H$, which defines the tensor product $\mathrm{St}_{H}$ of the two Steinberg representations.

Proposition 3.13. For any $f \in C_{c}^{\infty}\left(G\left(F_{v_{i}}\right)\right)$, we have

$$
\left\langle\operatorname{tr} Y_{i}-\operatorname{tr} X_{i}, f\right\rangle=\left\langle\epsilon_{i}\left(X_{i}\right) \operatorname{tr} X_{i}+\epsilon_{i}\left(Y_{i}\right) \operatorname{tr} Y_{i}, f\right\rangle=\left\langle\operatorname{tr} \operatorname{St}_{H}, f^{H}\right\rangle .
$$

Before sketching the proof we note that this is plausible. One property of the signs $\epsilon_{i}$ (for the Whittaker normalization) is that we should have $\epsilon(Z)=1$ when $Z$ is the representation in the L-packet for $G$ having a Whittaker model. The computation of the Jacquet modules shows that $Y_{i}$ is the "bigger" representation in the L-packet. Presumably it has a Whittaker model, although we have not checked this.

Proof. We sketch the proof in the case $n=6$; it will be clear that the proof extends. For the duration of this proof we also simplify notation by removing the dependence of the various objects on the subscript $i$. Thus

$$
\begin{gathered}
X_{N_{0}}^{\text {norm }}=[3,1,-1] \\
\left(Y_{N_{0}}^{\text {norm }}\right)^{\mathrm{ss}}=[3,1,-1]+2[3,1,1]+[1,3,1] .
\end{gathered}
$$

Let $T_{0}=Z_{U_{n}}(S)$ denote the maximal torus consisting of elements

$$
\operatorname{diag}\left(t_{1}, t_{2}, t_{3},{\overline{t_{3}}}^{-1},{\overline{t_{2}}}^{-1},{\overline{t_{1}}}^{-1}\right), t_{i} \in E^{\times} .
$$

The torus $T_{0}$ (or a stably conjugate torus in $H$ ) has trivial Galois cohomology, so the relation between $f$ and $f^{H}$ on elements conjugate to this torus is simply:

$$
O_{\delta}\left(f^{H}\right)=\Delta(\delta, \gamma) O_{\gamma}(f)
$$

( $\delta \in H, \gamma \in G$ regular semisimple and associated).

We already know, thanks to Mok's results [Mok, Theorem 3.2.1], that such an identity exists: only the signs of $X_{i}$ and $Y_{i}$ have to be determined. Thus we can choose $f$ to be supported on the $G$-conjugates of the following subset of $T_{0}$ :

$$
T_{0}^{+}=\left\{\left(t_{1}, t_{2}, t_{3}\right)|| t_{1}|<| t_{2}|<| t_{3} \mid<1\right\} .
$$

Similarly in $H$ we have

$$
T_{0}^{+}(H)=\left\{\left(t_{1}, t_{2}, t_{3}\right)|| t_{1}|<1,| t_{2}|<| t_{3} \mid<1\right\} .
$$

Assume that $f$ is such a function on a quasi-split group $G^{\prime}$ (which may be $G$ or $H$ ), and let $\pi$ be an admissible representation of $G^{\prime}$. The identity [Clo90a, (2.4)] (note that $G$ need not be unramified there) yields

$$
\langle\operatorname{tr} \pi, f\rangle=\int_{t \in T_{0}^{+}} \Theta\left(\pi_{N_{0}}^{\text {norm }}\right)(t) D(t) O_{t}(f) d t .
$$


Here $\pi_{N_{0}}^{\text {norm }}=\pi_{N_{0}} \delta_{P_{0}}^{-1 / 2}$ is the normalized Jacquet module, $\Theta\left(\pi_{N_{0}}^{\text {norm }}\right)$ is its trace, and

$$
D(t)=\prod_{\alpha>0}\left|t^{\alpha / 2}-t^{-\alpha / 2}\right| .
$$

Indeed, we first have by [Clo90a, (2.4)]

$$
\langle\operatorname{tr} \pi, f\rangle=\int_{t \in T_{0}^{+}} \Theta\left(\pi_{N_{0}}^{\text {norm }}\right) \bar{f}^{P_{0}}(t) d t
$$

where $\bar{f}^{P_{0}}(t)=\delta_{P_{0}}^{1 / 2}(t) \int_{N_{0}} \bar{f}(t n) d n$ and $\bar{f}$ is the conjugation $K$-average of $f$ for a good, maximal compact subgroup $K$ of $G$. By a formula of Harish-Chandra (see e.g. Car79, Lemma 4.1], and make the change of variable $\left.n \rightarrow t n t^{-1}\right)$ :

$$
\bar{f}^{P_{0}}(t)=\delta_{P_{0}}^{-1 / 2}(t) \Delta(t) O_{t}(f)
$$

for $t$ regular in $T_{0}$, where $\Delta(t)=\left|\operatorname{det}\left(1-\operatorname{Ad}_{\mathfrak{n}_{0}}(t)\right)\right|$. Now

$$
\delta_{P_{0}}^{-1 / 2}(t) \Delta(t)=\prod_{\alpha>0}\left|t^{-\alpha / 2}\right|\left|1-t^{\alpha}\right|=\prod_{\alpha>0}\left|t^{-\alpha / 2}-t^{\alpha / 2}\right|=D(t) .
$$

We first apply this to $G$, giving for such functions:

$$
\langle\operatorname{tr} Y-\operatorname{tr} X, f\rangle=\int_{t \in T_{0}^{+}}\left(2 e_{1}+e_{2}\right)(t) D_{G}(t) O_{t}(f) d t,
$$

where $e_{1}=[3,1,1]$ and $e_{2}=[1,3,1]$ are characters of $T_{0}$.

Consider now $f^{H}$ on $H$. The exponent of the normalized Jacquet module of $\mathrm{St}_{H}=\mathrm{St}_{U(2)} \otimes \mathrm{St}_{U(4)}$ is, with the same notation, $e=[1 ; 3,1]$. The orbital integrals of $f^{H}$ need not be supported in $T_{0}^{+}(H)$. However, we can write $f^{H}=\sum_{w} \chi_{w} f^{H}=\sum f_{w}^{H}$, where $w \in W\left(H, T_{0}\right)$ and $\chi_{w}$ is the characteristic function of the set of elements contracting $w \cdot N_{0}$. An easy calculation then shows that the formula

$$
\left\langle\operatorname{trSt}_{H}, f^{H}\right\rangle=\int_{t \in T_{0}^{+}(H)} e(t) D_{H}(t) O_{t}\left(f^{H}\right) d t
$$

remains true (note that $D_{H}(t)$ is invariant). Now if $t=\left(t_{1}, t_{2}, t_{3}\right) \in T_{0}^{+}$then it has three distinct conjugates in $T_{0}^{+}(H)$, namely

$$
\left(t_{1}, t_{2}, t_{3}\right),\left(t_{2}, t_{1}, t_{3}\right), \text { and }\left(t_{3}, t_{1}, t_{2}\right) .
$$

Assuming the identity

$$
D_{H}\left(t_{H}\right) O_{t_{H}}\left(f^{H}\right)=D_{G}(t) O_{t}(f)
$$

we then see that

$$
\left\langle\operatorname{trSt}_{H}, f^{H}\right\rangle=\int_{t \in T_{0}^{+}}\left(2 e_{1}+e_{2}\right)(t) D_{G}(t) O_{t}(f) d t,
$$

since $e\left(t_{1}, t_{2}, t_{3}\right)=e_{2}(t)$ and $e\left(t_{2}, t_{1}, t_{3}\right)=e\left(t_{3}, t_{1}, t_{2}\right)=e_{1}(t)$. Thus we have to check the identity

$$
D_{H}\left(t_{H}\right) O_{t_{H}}\left(f^{H}\right)=D_{G}(t) O_{t}(f),
$$

where $f, f^{H}$ are related by the identity $O_{t_{H}}\left(f^{H}\right)=\Delta\left(t_{H}, t\right) O_{t}(f)$. Recall that

$$
\Delta\left(t_{H}, t\right)=\Delta_{I} \Delta_{I I} \Delta_{I I I, 1} \Delta_{I I I, 2} \Delta_{I V}
$$

where $\Delta_{I V}$ is simply $D_{G}(t) / D_{H}\left(t_{H}\right)$. We check that the other factors are equal to 1 , for the LanglandsShelstad transfer factors (quasi-split case) of [LS87, which coincide with the Whittaker-normalized transfer factors in our case, cf. 3.5 . 
The factors $\Delta_{I}$ [LS87, p. 241] and $\Delta_{I I I, 1}$ [LS87, p. 245] are defined by a cup-product with $H^{1}\left(F, T_{\mathrm{sc}}\right)$; here $T=T_{0}, T_{\mathrm{sc}}=T \cap S U_{6}$ and $H^{1}\left(F, T_{\mathrm{sc}}\right)=\{1\}$ (apply Hilbert 90 twice). The factor $\Delta_{I I}$ is defined in [LS87, p. 243]. It requires the choice of data $a_{\alpha} \in E^{\times}$and $\chi_{\alpha}$ (a character of $E^{\times}$) for the roots $\alpha$ of $\left(G, T_{0}\right)$. In our case it is easily checked that $a_{\alpha}=1, \chi_{\alpha}=1$ are suitable and then $\Delta_{I I}=1$, by definition.

The term $\Delta_{I I I, 2}$ [LS87, p. $246-247$ ] is ostensibly the most complicated. However [LS87, Definition p. 247], we have

$$
\Delta_{I I I, 2}\left(t_{H}, t\right)=\chi\left(t_{H}\right),
$$

where $\chi$ is a character of $T_{0}(H)$ defined by Langlands functoriality for tori. Here $\chi$ is defined by an element $a \in H^{1}\left(W_{F}, \widehat{T_{0}}\right)$ where we have written $F=F_{v_{i}}$. In turn $a$ is obtained by comparing the (tautological) embedddings ${ }^{L} T_{0} \rightarrow{ }^{L} G,{ }^{L} T_{0}(H) \rightarrow{ }^{L} H$ and the natural embedding ${ }^{L} H \rightarrow{ }^{L} G$, cf. 2.2. In our case one finds that $a$ is trivial. This completes the proof of Proposition 3.13.

\subsection{Transfer}

We now note that by Lab11a, Proposition 5.6] there is an identity

$$
T_{\mathrm{disc}}^{G}(f)=\sum_{\mathcal{E}} \iota(G, \mathcal{E}) S T_{\mathrm{disc}}^{\mathcal{E}}\left(f^{\mathcal{E}}\right)
$$

for $f, f^{H}$ which are associated, the sum being over the elliptic endoscopic data of $G$. The terms $S T_{\text {disc }}^{\mathcal{E}}\left(f^{\mathcal{E}}\right)$ have been computed by Mok, cf. [Mok, Theorem 5.1.2]. If $f=\otimes_{v} f_{v}=f^{\infty} f_{\infty}=f^{S} f_{S}$ is chosen so that $f_{\infty}$ is an Euler-Poincaré function for the trivial representation of $G\left(F_{\infty}\right)$ (e.g. the constant function), then $f$ traces in only finitely many automorphic representations of $G$. By a separation of eigenvalues argument (cf. [CHL11, p. 487]), we can even choose $f^{S}$ so that the only non-trivial contributions in the above formula come from the groups $G^{*}$ and $H$, and only the parameter $\psi$ contributes in the expression for the stable trace of [Mok, Theorem 5.1.2].

Let us first suppose that the parameter $\psi$ corresponds to a RACSDC automorphic representation of $\mathrm{GL}_{n}\left(\mathbb{A}_{E}\right)$, as in the second part of Theorem 3.11. In this case we obtain a formula (Mok's notation):

$$
T_{\mathrm{disc}}^{G}(f)=f^{G^{*}}(\psi)=\prod_{v \nmid \infty} f_{v}^{G^{*}}\left(\psi_{v}\right) .
$$

(Strictly speaking we use here the analogue of Lemma 3.12 for the endoscopic group $G^{*}$ of $G$, which states that $\Delta_{v}\left(\psi_{v, G^{*}}, \sigma_{v}\right)=1$ for each infinite place $v$ of $F$.) The theorem now follows in this case from the identity

$$
f_{v_{i}}^{G}\left(\psi_{v_{i}}\right)=\left\langle\operatorname{tr} X_{i}+\operatorname{tr} Y_{i}, f_{v_{i}}\right\rangle
$$

when $\pi_{w_{i}} \cong \mathrm{St}_{2, E_{w_{i}}} \boxplus \mathrm{St}_{n-2, E_{w_{i}}}$. The third part of Theorem 3.11 follows in a similar manner, taking into account that the L-packet of representations of $G\left(F_{v_{0}}\right)$ corresponding to the representation $\mathrm{St}_{n, E_{w_{0}}}(\psi)$ of $\mathrm{GL}_{n}\left(E_{w_{0}}\right)$ contains a single element. Note that there is a similar identity, relating twisted trace and trace, for the Steinberg representations of $U(n)$ and of $G L\left(n, E_{w}\right)$.

Now suppose that the parameter $\psi$ corresponds to a sum $\pi=\pi_{1} \boxplus \pi_{2}$ of RACSDC automorphic representations of $\mathrm{GL}_{2}\left(\mathbb{A}_{E}\right)$ and $\mathrm{GL}_{n-2}\left(\mathbb{A}_{E}\right)$, respectively, such that $\pi$ is $\iota$-ordinary and regular algebraic of weight zero, as in the first part of Theorem 3.11. It follows that $\pi_{1}$ and $\pi_{2}$ are $\iota$-ordinary and the infinity types $\mathbf{a}, \mathbf{b}$ satisfy the following condition (cf. Lemma 3.5):

- Let $\mathbf{w}=\left(w_{\tau}\right)_{\tau: E \hookrightarrow \mathbb{C}} \in \mathfrak{S}_{n}^{\operatorname{Hom}(E, \mathbb{C})}$ be the tuple of permutations associated to $\pi_{1}, \pi_{2}$, cf. 3.1 . Then $w_{\tau}$ depends only on the place of $E$ induced by the embedding $\iota^{-1} \tau: E \hookrightarrow \overline{\mathbb{Q}}_{l}$.

Choose for each place $v$ of $F$ dividing $l$ an embedding $\tau(v): E \hookrightarrow \mathbb{C}$ such that $\iota^{-1} \tau$ induces the place $v$. We have by Lemma 3.12 a formula

$$
\prod_{v \mid \infty} \Delta\left(\psi_{v, H}, \sigma_{v}\right)=\prod_{v \mid l} \operatorname{det} w_{\tau(v)}^{\left[F_{v}: \mathbb{Q}_{l}\right]}
$$


Since the local degrees $\left[F_{v}: \mathbb{Q}_{l}\right]$ are even by hypothesis, this product is equal to 1 and we obtain a formula

$$
T_{\text {disc }}^{G}(f)=\left(f^{G^{*}}(\psi)+f^{H}\left(\psi_{H}\right)\right) / 2=\left(\prod_{v \nmid \infty} f_{v}^{G}\left(\psi_{v}\right)+\prod_{v \nmid \infty} f_{v}^{H}\left(\psi_{v, H}\right)\right) / 2 .
$$

The contribution from the places $v_{0}, \ldots, v_{s}$ is (by Proposition 3.13):

$$
\frac{1}{2}\left(\prod_{i=0}^{s} f_{v_{i}}^{G}\left(\psi_{v_{i}}\right)+\prod_{i=0}^{s} f_{v_{i}}^{H}\left(\psi_{v_{i}, H}\right)\right)=\sum_{\mathbf{d}}\left[\frac{1}{2}\left(1+\prod_{i=0}^{s} \epsilon_{i}\left(d_{i}\right)\right) \prod_{i=0}^{s}\left\langle\operatorname{tr} d_{i}, f_{v_{i}}\right\rangle\right],
$$

where the notation $\mathbf{d}$ is as in the statement of the theorem. This completes the proof.

\section{Raising the level}

Let $E$ be an imaginary CM field with totally real subfield $F$. We fix a prime $l \geq 5$ and an isomorphism $\iota: \overline{\mathbb{Q}}_{l} \cong \mathbb{C}$. Let $n=l+1$. We make the following hypotheses:

- For each place $v \mid l$ of $F, v$ splits in $E$ and $\left[F_{v}: \mathbb{Q}_{l}\right]$ is even. In particular, $[F: \mathbb{Q}]$ is even and there exists a unitary group $G$ in $n$ variables over $F$ such that $G\left(F_{\infty}\right)$ is compact and $G$ is quasi-split at every finite place.

- Let $v_{0}, \ldots, v_{s}$ be the places of $F$ ramified in $E$. Then $s \geq 1, q_{v_{0}} \equiv-1 \bmod l$ and for each $i=1, \ldots, s, l$ does not divide $q_{v_{i}}\left(q_{v_{i}}+1\right) \prod_{j=1}^{n / 2-2}\left(q_{v_{i}}^{j}-1\right)$. (This will be the case if, for example, $q_{v_{i}}$ is a primitive root modulo $l$.)

We write $w_{0}, \ldots, w_{s}$ for the places of $E$ above $v_{0}, \ldots, v_{s}$. We fix RACSDC automorphic representations $\pi_{2}$, $\pi_{l-1}$ of $\mathrm{GL}_{2}\left(\mathbb{A}_{E}\right)$ and $\mathrm{GL}_{l-1}\left(\mathbb{A}_{E}\right)$, respectively, satisfying the following hypotheses:

- $\pi_{2}$ and $\pi_{l-1}$ are $\iota$-ordinary. For each embedding $\tau: E \hookrightarrow \mathbb{C}$, the infinity type of $\pi_{l-1}$ at $\tau$ is

$$
((n-3) / 2,(n-5) / 2, \ldots,(5-n) / 2,(3-n) / 2)
$$

and the infinity type of $\pi_{2}$ at $\tau$ is

$$
((n-1) / 2,(1-n) / 2)
$$

- The residual representations $\bar{r}_{2}=\overline{r_{\iota}\left(\pi_{2}\right)}$ and $\bar{r}_{l-1}=\overline{r_{\iota}\left(\pi_{l-1}\right)}$ are irreducible.

- For each $i=0, \ldots, s$ we have isomorphisms

$$
\pi_{2, w_{i}} \cong \mathrm{St}_{2, E_{w_{i}}} \text { and } \pi_{l-1, w_{i}} \cong \mathrm{St}_{l-1, E_{w_{i}}} \text {. }
$$

The residual representations $\left.\bar{r}_{2}\right|_{G_{E_{w_{i}}}}$ and $\left.\bar{r}_{l-1}\right|_{G_{E_{w_{i}}}}$ are ramified and send a generator of tame inertia at each of these places to a regular unipotent element (that is, having a single Jordan block).

- Any finite place $w \neq w_{0}, \ldots, w_{s}$ of $E$ at which $\pi_{2}$ or $\pi_{l-1}$ is ramified is split over $F$.

In this section we intend to prove the following theorem.

Theorem 4.1. With hypotheses as above, there exists a RACSDC automorphic representation $\Pi$ of $\mathrm{GL}_{n}\left(\mathbb{A}_{E}\right)$ which is $\iota$-ordinary of weight 0 , such that $\overline{r_{\iota}(\Pi)} \cong \overline{r_{\iota}\left(\pi_{2} \boxplus \pi_{l-1}\right)}$, and such that $\Pi_{w_{0}}$ is an unramified twist of the Steinberg representation.

In $₫ 2.2$ we introduced $L$-packets $\left\{X_{i}, Y_{i}\right\}$ of representations of the groups $G\left(F_{v_{i}}\right)$ corresponding to the representations $\mathrm{St}_{2, E_{w_{i}}} \boxplus \mathrm{St}_{l-1, E_{w_{i}}}$ of $\mathrm{GL}_{n}\left(E_{w_{i}}\right)$. Let $\mathfrak{B}_{v_{i}}$ denote an Iwahori subgroup of $G\left(F_{v_{i}}\right)$. Then these representations are characterized within their L-packet by the equations $\operatorname{dim} X_{i}^{\mathfrak{B}_{v_{i}}}=1, \operatorname{dim} Y_{i}^{\mathfrak{B}_{v_{i}}}=n / 2+1$, cf. Theorem 2.7. By Theorem 3.11. there exists an automorphic representation $\sigma_{0}$ of $G\left(\mathbb{A}_{F}\right)$ with base change $\pi_{2} \boxplus \pi_{l-1}$. We observe that $\sigma_{0}$ is $\iota$-ordinary, by Lemma 3.5 .

We define an open compact subgroup $U_{1}=\prod_{v} U_{1, v}$ of $G\left(\mathbb{A}_{F}^{\infty}\right)$ as follows: 
- $U_{1, v_{0}}=\mathfrak{P}_{v_{0}}$, the subgroup containing the Iwahori subgroup defined in $\$ 2.1$

- For each $i=1, \ldots, s, U_{1, v_{i}}=\mathfrak{B}_{v_{i}}$.

- For each place $v \mid l$ of $F$, choose a place $\widetilde{v}$ of $E$ above it, and set $U_{1, v}=\iota_{\widetilde{v}}^{-1} \operatorname{Iw}_{c}(\widetilde{v})$ for some integer $c>0$.

- For each place $v$ of $F$ inert in $E, U_{1, v}$ is a hyperspecial maximal compact subgroup of $G\left(F_{v}\right)$.

- For some place $v, U_{1, v}$ contains no non-trivial elements of finite order. (This condition is sometimes referred to by saying that $U_{1}$ is sufficiently small.)

$-\sigma_{0}^{U_{1}} \neq 0$.

We define another open compact subgroup $U=\prod_{v} U_{v}$ by the formulae $U_{v_{0}}=\mathfrak{B}_{v_{0}}$ and $U_{v}=U_{1, v}$ if $v \neq v_{0}$. Thus $U \subset U_{1}$ and $\left[U_{1}: U\right]=\left[U_{1, v_{0}}: U_{v_{0}}\right]=q_{v_{0}}+1$.

Fix a finite extension $K \subset \overline{\mathbb{Q}}_{l}$ of $\mathbb{Q}_{l}$, with ring of integers $\mathcal{O}$, residue field $k$, and maximal ideal $\lambda$. We write $S\left(U_{1}, \mathcal{O}\right)$ for the space of automorphic forms on $G$ with trivial coefficients and level $U_{1}$, as defined in $\$ 3.3$. Let $T$ denote the set of finite places of $F$ above which $E$ or $\pi$ is ramified or such that $U_{1, v}$ is not hyperspecial maximal compact. (Thus $T$ contains the places dividing $l$.) We then define the Hecke algebra $\mathbb{T}\left(U_{1}, \mathcal{O}\right)$ to be the $\mathcal{O}$-subalgebra of $\operatorname{End}_{\mathcal{O}}\left(S\left(U_{1}, \mathcal{O}\right)\right)$ generated by the unramified Hecke operators at places of $F$ not in $T$ and split in $E$, and the operators $U_{v}^{j}$ for each $v \mid l$. It is a finite flat $\mathcal{O}$-algebra. (We recall that the definition of $U_{v}^{j}$ depends on a choice of place $\widetilde{v}$ of $E$ above $v$ and a uniformizer of $E_{\widetilde{v}}$, but these choices play no role here.)

The representation $\sigma_{0}$ gives rise to a homomorphism $\mathbb{T}\left(U_{1}, \mathcal{O}\right) \rightarrow \overline{\mathbb{F}}_{l}$, and we write $\mathfrak{m}$ for the kernel of this homomorphism. Then $S\left(U_{1}, \mathcal{O}\right)_{\mathfrak{m}}$ is an $\mathcal{O}$-direct summand of $S\left(U_{1}, \mathcal{O}\right)$, and every automorphic representation $\sigma$ of $G\left(\mathbb{A}_{F}\right)$ which contributes to $S\left(U_{1}, \mathcal{O}\right)_{\mathfrak{m}}$ is $\iota$-ordinary. Moreover, $\mathbb{T}\left(U_{1}, \mathcal{O}\right)_{\mathfrak{m}} \otimes_{\mathcal{O}} K$ is a semisimple $K$-algebra.

Now suppose that $V=\prod_{v} V_{v} \subset U_{1}$ is an open compact subgroup such that for each place $v$ of $F$ such that either $v \mid l$ or $v \notin T, V_{v}=U_{1, v}$. We can define the space $S(V, \mathcal{O})$ and Hecke algebra $\mathbb{T}(V, \mathcal{O})$ and a natural surjective homomorphism $\mathbb{T}(V, \mathcal{O}) \rightarrow \mathbb{T}\left(U_{1}, \mathcal{O}\right)$. In an abuse of notation, we will write $\mathfrak{m}$ also for the pullback of this maximal ideal to $\mathbb{T}(V, \mathcal{O})$.

Using Theorem 3.11, we see that there is a direct sum decomposition

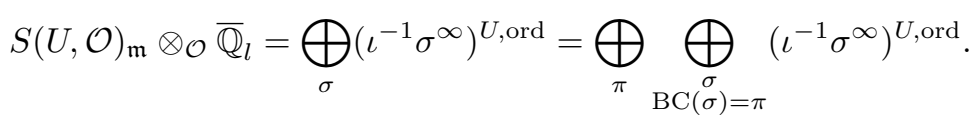

Here the first sum in the third term runs over automorphic representations $\pi$ of $\mathrm{GL}_{n}\left(\mathbb{A}_{E}\right)$. The second, inner, sum in the third term runs over automorphic representations $\sigma$ of $G\left(\mathbb{A}_{F}\right)$ which contribute to $S(U, \mathcal{O})_{\mathfrak{m}}$ and such that $\pi$ is the base change of $\sigma$. We will say that a representation $\pi$ for which the $\pi$-summand in the above expression is non-trivial is relevant.

Proposition 4.2. Let $\pi$ be relevant. Then one of the following is true:

- $\pi=\pi_{a} \boxplus \pi_{b}$, where $\pi_{a}, \pi_{b}$ are RACSDC automorphic representations of $\mathrm{GL}_{2}\left(\mathbb{A}_{E}\right), \mathrm{GL}_{l-1}\left(\mathbb{A}_{E}\right)$, respectively, and for each $i=0, \ldots, s, \pi_{w_{i}} \cong \mathrm{St}_{2, E_{w_{i}}} \boxplus \mathrm{St}_{l-1, E_{w_{i}}}$.

- $\pi$ is cuspidal and for each $i=0, \ldots, s, \pi_{w_{i}} \cong \mathrm{St}_{2, E_{w_{i}}} \boxplus \mathrm{St}_{l-1, E_{w_{i}}}$.

- $\pi$ is cuspidal and $\pi_{w_{0}}$ is an unramified twist of the Steinberg representation, and for each $i=$ $1, \ldots, s, \pi_{w_{i}} \cong \mathrm{St}_{2, E_{w_{i}}} \boxplus \mathrm{St}_{l-1, E_{w_{i}}}$.

Proof. Let $\pi$ be as in the statement of the theorem. By Proposition 3.7. we can write $\pi \cong \pi_{1} \boxplus \cdots \boxplus \pi_{r}$, where the $\pi_{i}$ are discrete and conjugate self-dual automorphic representations of $\mathrm{GL}_{n_{i}}\left(\mathbb{A}_{F}\right)$. Let $\rho=r_{\iota}(\pi)$. Then $\bar{\rho}$ is a direct sum of two irreducible representations of distinct dimensions. In particular, we must have either $r=1$ and $\pi$ is cuspidal, or $r=2, n_{1}=2, n_{2}=n-2=l-1$ and $\pi_{1}$ and $\pi_{2}$ are both cuspidal. In this case $\pi_{1}$ and $\pi_{2}$ are also regular algebraic. We now apply the following.

Lemma 4.3. Let $\pi$ be relevant. Then for each $i=0, \ldots, s, \pi_{w_{i}}$ has an Iwahori-fixed vector.

Proof. We fix $i$ to be one of $0, \ldots, s$ for the duration of the proof. Assume first that $\pi$ is cuspidal. By the identity at the beginning of 33.8 and [Lab11a, Theorem 4.12] we obtain, after separation of Hecke eigenvalues:

$$
\sum_{\sigma}\langle\operatorname{tr} \sigma, f\rangle=\left\langle\operatorname{tr} \pi_{v_{0}} \otimes \cdots \otimes \pi_{v_{s}} \otimes \pi_{\infty} \times I_{c}, f_{E}\right\rangle
$$


Here $f$ is a function on $G\left(F_{\infty} \times \prod_{i=0}^{s} F_{v_{i}}\right), f_{E}$ is a function on $G\left(E_{\infty} \times \prod_{i=0}^{s} E_{w_{i}}\right)$ associated to $f$ by stable base change, and $\sigma$ runs over the local components of automorphic representations of $G\left(\mathbb{A}_{F}\right)$ associated to $\pi$. We may further assume that $f_{\infty}=1$. (If we use Mok's full results then the sum is finite, each term occurring with multiplicity one, since the same identity obtains for $G^{*}$, isomorphic to $G$ at the finite places. We do not need this.)

Now fix $v=v_{i}, w=w_{i}$ for some $i=0, \ldots, s$. Choosing the functions for $v^{\prime} \neq v$ suitably we obtain

$$
c \sum_{\sigma}\left\langle\operatorname{tr} \sigma, f_{v}\right\rangle=\left\langle\operatorname{tr} \pi_{w} \times I_{c}, f_{E_{w}}\right\rangle,
$$

with $c \neq 0$. The representation of $G\left(\mathbb{A}_{F}\right)$ is admissible, so the left-hand side contains a finite number of semistable representations $\sigma$ with finite multiplicity. (A semistable representation is, by definition, one that has a non-zero Jacquet module for $N_{0}$, composed of unramified characters.)

Consider a function $f_{v}$ with support in the elements contracting $N_{0}$. We may further assume that the constant term $\bar{f}^{P_{0}}(t)$ (cf. 3.7 is an unramified function. By the descent formula for the traces (\$3.7), the left-hand side is then a finite sum, over the semistable representations:

$$
c \sum_{\sigma}\left\langle\operatorname{tr} \sigma_{N_{0}}^{\text {norm }}, \bar{f}^{P_{0}}\right\rangle_{T_{0}}
$$

By assumption, the sum contains a representation $\sigma_{0}$ such that $\left(\sigma_{0}\right)_{N_{0}}$ is a sum of unramified characters; note that there is no cancellation in the sum. However, the identity of orbital integrals shows that we can take for $f_{E_{w}}$ a function whose orbital integrals have the same property. The right-hand side of the identity is then equal to

$$
\left\langle\operatorname{tr} \pi_{N_{0}(E)}^{\text {norm }},\left(\overline{f_{E_{w}}}\right)^{P_{0}}\right\rangle
$$

and this implies that $\pi_{w}$ is semistable. (Moeglin Mœe07 shows that in fact the resulting identity of Jacquet modules extends from the contracting elements to all of $T_{0}$ and $T_{0}(E)$.)

Consider now the case where $\pi=\pi_{2} \boxplus \pi_{n-2}$ with $\pi_{2}, \pi_{n-2}$ cuspidal. In this case the relevant equality is given by 3.8 . The sum $\sum_{\sigma}\langle\operatorname{tr} \sigma, f\rangle$ is equal to the sum of two terms, one pertaining to $G^{*}$ :

$$
1 / 2\left\langle\operatorname{tr} \pi \times I_{c}, f_{E}\right\rangle,
$$

where however $\pi$ is an Eisenstein representation $\pi=\pi_{2} \boxplus \pi_{n-2}$. This is the term (4.4.2) in [CHL11; the proof is identical. The second term is

$$
1 / 2\left\langle\operatorname{tr}\left(\pi_{2} \otimes \pi_{n-2}\right) \times I_{c}, f_{E}^{H}\right\rangle,
$$

where $H=U(2) \times U(n-2)$ is the endoscopic group of our datum. We choose $f_{v}$ and the $f_{v^{\prime}}$ for $v^{\prime} \neq v$ as above, so the previous argument applies to $\sum_{\sigma}$, non-zero by assumption. If the first term does not vanish, the local component of $\pi_{2} \boxplus \pi_{n-2}$ at $v$ is semistable; it follows that the local components of $\pi_{2}, \pi_{n-2}$ at $v$ are also semistable. If the $H$-term does not vanish, the computation of the transfer in 3.7 shows that we may choose $f^{H}$ unramified, with regular support, thus also $f_{E}^{H}$, the transfer being obvious on the split torus. Again this implies that the local components of $\pi_{2}$ and $\pi_{n-2}$ at $v$ are semistable.

We now return to the proof of Proposition 4.2 Suppose first that $r=2$, so that $\rho=\rho_{a}(1-$ $n / 2) \oplus \rho_{b}(-1)$, where $\rho_{a}=r_{\iota}\left(\pi_{a}\right)$ and $\rho_{b}=r_{\iota}\left(\pi_{b}\right)$. The hypotheses on the residual representations $\bar{\rho}_{a} \cong \bar{r}_{2}$, $\bar{\rho}_{b} \cong \bar{r}_{l-1}$ now imply that for each $i=0, \ldots, s$, the representation $\pi_{a, w_{i}}$ (resp. $\pi_{b, w_{i}}$ ) is an unramified twist of $\mathrm{St}_{2, E_{w_{i}}}$ (resp. $\mathrm{St}_{l-1, E_{w_{i}}}$ ). Indeed, it is easy to see that since $\pi_{w_{i}}$ has an Iwahori-fixed vector, the same must be true for the representations $\pi_{a, w_{i}}$ and $\pi_{b, w_{i}}$. We therefore have, for example, an isomorphism

$$
\pi_{b, w_{i}} \cong \mathrm{St}_{b_{1}, E_{w_{i}}}\left(\psi_{1}\right) \boxplus \cdots \boxplus \mathrm{St}_{b_{t}, E_{w_{i}}}\left(\psi_{t}\right),
$$

where $b_{1}+\cdots+b_{t}=n-2=l-1$ and each $\psi_{1}, \ldots, \psi_{t}: E_{w_{i}}^{\times} \rightarrow \mathbb{C}^{\times}$is an unramified character. Let $t_{w_{i}} \in I_{w_{i}}$ denote a generator of the $l$-part of tame inertia. Local-global compatibility in its strong form (cf. Car12] ) now implies that $\rho_{b}\left(t_{w_{i}}\right)$ is a unipotent matrix with Jordan form corresponding to the partition 
$b_{1}+\cdots+b_{t}=l-1$. After conjugating and possibly enlarging $K$, we can assume that $\rho_{b}$ takes values in $\mathrm{GL}_{l-1}(\mathcal{O})$, and that the composite $G_{E} \rightarrow \mathrm{GL}_{l-1}(\mathcal{O}) \rightarrow \mathrm{GL}_{l-1}(k)$ is equal to $\bar{r}_{l-1}$. By hypothesis, $\bar{r}_{l-1}\left(t_{w_{i}}\right)$ is a regular unipotent matrix. It follows that we must have $t=1$, and then $\pi_{b, w_{i}}$ is an unramified twist of the Steinberg representation, as claimed. To see that the first bullet point holds in this situation, we must check that these twists are all actually trivial. To do this we look at the Frobenius eigenvalues of $\bar{\rho}_{a}$ and $\bar{\rho}_{b}$. Let us treat, for example, $\pi_{b, w_{0}} \cong \operatorname{St}_{l-1, E_{w_{0}}}(\psi)$. Since $\psi \psi^{c}=1$ (as $\pi_{b}$ is of unitary type) and $\psi=\psi^{c}$ (as $\psi$ is unramified), we see that $\psi^{2}=1$ and we must rule out the case that $\psi$ is the non-trivial unramified character of order 2 .

Let $\varpi_{w_{0}}$ be a uniformizer of $E_{w_{0}}$, and let $N=\rho_{b}\left(t_{w_{0}}\right)-1 \in M_{l-1}(\mathcal{O})$. Then $N \bmod \lambda$ is a regular nilpotent element, by hypothesis, and the natural map

$$
(\operatorname{ker} N) \otimes_{\mathcal{O}} k \rightarrow \operatorname{ker}(N \bmod \lambda)
$$

is an isomorphism. In particular, $\bar{\rho}_{b}$ preserves the line $\operatorname{ker}(N \bmod \lambda)$ and Frobenius acts with eigenvalue

$$
\iota^{-1} \psi\left(\varpi_{w_{0}}\right) \epsilon\left(\text { Frob }_{w_{0}}\right)^{-1} q_{w_{0}}^{l-2} \equiv \iota^{-1} \psi\left(\varpi_{w_{0}}\right) \bmod \lambda .
$$

Since $\pi_{l-1, w_{0}}$ is, by hypothesis, the untwisted Steinberg representation, performing the same calculation for $\bar{r}_{l-1}$ gives $\iota^{-1} \psi\left(\varpi_{w_{0}}\right) \equiv 1 \bmod \lambda$, and hence $\psi=1$.

Now suppose that $r=1$, so that $\pi$ is cuspidal. Let $0 \leq i \leq s$. Since $\pi_{w_{i}}$ has Iwahori-fixed vectors, there is an isomorphism

$$
\pi_{w_{i}} \cong \mathrm{St}_{n_{1}, E_{w_{i}}}\left(\psi_{1}\right) \boxplus \cdots \boxplus \mathrm{St}_{n_{t}, E_{w_{i}}}\left(\psi_{t}\right)
$$

for some $t \geq 1$, where the $\psi_{i}$ are unramified characters of $E_{w_{i}}^{\times}$. The congruence $\bar{\rho} \cong \overline{r_{\iota}\left(\sigma_{0}\right)}$ implies that the nilpotent conjugacy class of $\mathrm{GL}_{n}$ corresponding to the partition $n=n_{1}+\cdots+n_{t}$ specializes to the class corresponding to the partition $2+(n-2)$. This rules out all but the possibilities $n=2+(n-2)$, $n=1+(n-1)$, and $n=n$. We must rule out the case $n=1+(n-1)$ and show that in case $n=2+(n-2)$ the characters $\psi_{1}, \psi_{2}$ are trivial, and that in case $n=n$ we necessarily have $i=0$. This will complete the proof of the proposition.

To rule out the case $n=1+(n-1)$, we note that no representation $\psi_{1} \boxplus \operatorname{St}_{n-1, E_{w_{i}}}\left(\psi_{2}\right)$ with $\psi_{1}, \psi_{2}$ unramified is in the image of the stable base change map, as the corresponding parameter is not conjguate symplectic, cf. Lemma 2.6. Suppose instead that $\pi_{w_{i}} \cong \operatorname{St}_{2, E_{w_{i}}}\left(\psi_{1}\right) \boxplus \operatorname{St}_{l-1, E_{w_{i}}}\left(\psi_{2}\right)$. After conjugating, we may assume that $\rho$ takes values in $\operatorname{GL}_{n}(\mathcal{O})$ and that $\rho \bmod \lambda$ is semisimple. Let $N=\rho\left(t_{w_{i}}\right)-1$. For each $j \geq 0$ the natural map $\left(\operatorname{ker} N^{j}\right) \otimes_{\mathcal{O}} k \rightarrow \operatorname{ker}\left(N^{j} \bmod \lambda\right)$ is an isomorphism and comparing the eigenvalues of Frobenius on $\operatorname{ker}(N \bmod \lambda)$ of $\bar{\rho}$ and $\bar{r}_{2}(1-n / 2) \oplus \bar{r}_{l-1}(-1)$, we get

$$
\iota^{-1} \psi_{1}\left(\varpi_{w_{i}}\right) q_{w_{i}}^{n / 2} \equiv q_{w_{i}}^{n / 2} \bmod \lambda \text { and } \iota^{-1} \psi_{2}\left(\varpi_{w_{i}}\right) q_{w_{i}}^{l-1} \equiv q_{w_{i}}^{l-1} \bmod \lambda .
$$

We again have $\psi_{1}^{2}=\psi_{2}^{2}=1$. It follows that $\psi_{1}$ and $\psi_{2}$ are both trivial. Finally, suppose that we have $\pi_{w_{i}} \cong \operatorname{St}_{n, E_{w_{i}}}(\psi)$ for some unramified character $\psi: E_{w_{i}}^{\times} \rightarrow \mathbb{C}^{\times}$. Comparing the Frobenius eigenvalues at $w_{i}$ of $\rho$ and $r_{\iota}\left(\sigma_{0}\right)$ shows that

$$
\iota^{-1} \psi\left(\varpi_{w_{i}}\right)\left\{q_{w_{i}}^{l}, q_{w_{i}}^{l-1}, \ldots, q_{w_{i}}, 1\right\} \equiv\left\{q_{w_{i}}^{n / 2}, q_{w_{i}}^{n / 2-1}, q_{w_{i}}^{l-1}, q_{w_{i}}^{l-2}, \ldots, q_{w_{i}}\right\} \bmod \lambda,
$$

where again $\psi\left(\varpi_{w_{i}}\right)= \pm 1$. Suppose for contradiction that $i>0$. If $\psi\left(\varpi_{w_{i}}\right)=1$ then the above equality of sets of eigenvalues cannot hold, since $q_{w_{i}}^{n / 2-1}=q_{w_{i}}^{(l-1) / 2} \equiv-1 \bmod l$. If $\psi\left(\varpi_{w_{i}}\right)=-1$, then the injection $\left(\operatorname{ker} N^{3}\right) \otimes_{\mathcal{O}} k \hookrightarrow \operatorname{ker}\left(N^{3} \bmod \lambda\right)$ shows that

$$
\left\{-q_{w_{i}}^{l},-q_{w_{i}}^{l-1},-q_{w_{i}}^{l-2}\right\} \bmod \lambda \subset\left\{q_{w_{i}}^{n / 2}, q_{w_{i}}^{n / 2-1}, q_{w_{i}}^{l-1}, q_{w_{i}}^{l-2}, q_{w_{i}}^{l-3}\right\} \bmod \lambda,
$$

or equivalently

$$
\left\{-q_{w_{i}},-1,-1 / q_{w_{i}}\right\} \bmod \lambda \subset\left\{-q_{w_{i}},-1,1,1 / q_{w_{i}}, 1 / q_{w_{i}}^{2}\right\} \bmod \lambda .
$$

It follows that $-1 / q_{w_{i}} \bmod \lambda \in\left\{1,1 / q_{w_{i}}, 1 / q_{w_{i}}^{2}\right\} \bmod \lambda$, again giving a contradiction. This completes the proof. 
Corollary 4.4. Let $\pi$ be relevant. Then each automorphic representation $\sigma$ of $G\left(\mathbb{A}_{F}\right)$ with base change $\pi$ and $\left(\sigma^{\infty}\right)^{U} \neq 0$ occurs with multiplicity one in the space of automorphic forms on $G$. Moreover, we have the following possibilities:

1. If $\pi$ is not cuspidal then there are exactly $2^{s}$ such representations $\sigma$. We index them by a choice of element $\mathbf{d}=\left(d_{1}, \ldots, d_{s}\right) \in \prod_{i=1}^{s}\left\{X_{i}, Y_{i}\right\}$. The corresponding automorphic representation $\sigma(\mathbf{d})$ is uniquely characterized by the condition

$$
\sigma(\mathbf{d})_{v_{i}} \cong d_{i}, i=1, \ldots, s
$$

It satisfies the condition

$$
\epsilon_{0}\left(\sigma(\mathbf{d})_{v_{0}}\right) \cdot \prod_{i=1}^{s} \epsilon_{i}\left(d_{i}\right)=1
$$

where $\epsilon_{i}:\left\{X_{i}, Y_{i}\right\} \rightarrow\{ \pm 1\}$ is defined by $\epsilon_{i}\left(X_{i}\right)=-1, \epsilon_{i}\left(Y_{i}\right)=1$.

2. If $\pi$ is cuspidal and $\pi_{w_{0}} \cong \mathrm{St}_{2, E_{w_{0}}} \boxplus \mathrm{St}_{l-1, E_{w_{0}}}$ then there are exactly $2^{\text {s+1 }}$ such representations, corresponding as above to a choice of element of $\prod_{i=0}^{s}\left\{X_{i}, Y_{i}\right\}$.

3. If $\pi$ is cuspidal and $\pi_{w_{0}}$ is an unramified twist of the Steinberg representation then there are exactly $2^{s}$ such representations, corresponding as above to a choice of element of $\prod_{i=1}^{s}\left\{X_{i}, Y_{i}\right\}$.

Proof. This follows from Proposition 4.2 and Theorem 3.11

This corollary has the following consequence. Let $\mathbf{d}=\left(X_{1}, \ldots, X_{s}\right)$ if $s$ is odd, and $\mathbf{d}=\left(Y_{1}, X_{2}, \ldots, X_{s}\right)$ is $s$ is even. Let $\pi$ be relevant, and suppose that $\pi$ is not cuspidal. Then $\sigma(\mathbf{d})_{v_{0}} \cong X_{0}$. We fix this choice of $\mathbf{d}$ for the remainder of this section. (In fact, any choice of $\mathbf{d} \in \prod_{i=1}^{s}\left\{X_{i}, Y_{i}\right\}$ with $\prod_{i=1}^{s} \epsilon_{i}\left(d_{i}\right)=-1$ would suffice for what follows.)

Now let $V \subset U$ be an open compact subgroup of the kind considered above. There is a pairing

$$
\langle\cdot, \cdot\rangle_{V}: S(V, \mathcal{O}) \times S(V, \mathcal{O}) \rightarrow \mathcal{O},
$$

given by the formula (for any two functions $f, g \in S(V, \mathcal{O})$ ):

$$
\langle f, g\rangle=\sum_{x \in G(F) \backslash G\left(\mathbb{A}_{F}^{\infty}\right) / V} f(x) g(x) .
$$

This is clearly a perfect pairing, and it moreover satisfies the formula $\left\langle\left[V_{v} g_{v} V_{v}\right] x, y\right\rangle=\left\langle x,\left[V_{v} g_{v}^{-1} V_{v}\right] y\right\rangle$ for any $g_{v} \in G\left(F_{v}\right), x, y \in S(V, \mathcal{O})$. There is a canonical isomorphism $S(V, \mathcal{O}) \otimes_{\mathcal{O}} k \cong S(V, k)$, and the induced pairing on $S(V, k)$ is still perfect. (These two assertions use that assumption that the open compact subgroup $U$ is sufficiently small.)

This pairing need not restrict to a perfect duality on $S(V, \mathcal{O})_{\mathfrak{m}}$. In fact, for any automorphic representation $\sigma$ of $G\left(\mathbb{A}_{F}\right)$ which contributes to $S(V, \mathcal{O})$, its admissible dual $\sigma^{\vee}$ also contributes. We write $\mathfrak{m}^{\vee} \subset \mathbb{T}(U, \mathcal{O})$ for the maximal ideal corresponding to the Hecke eigenvalues of $\sigma_{0}^{\vee}$. We have the following result.

Proposition 4.5. - - The above pairing restricts to a perfect duality

$$
\langle\cdot, \cdot\rangle_{V, \mathfrak{m}}: S(V, \mathcal{O})_{\mathfrak{m}} \times S(V, \mathcal{O})_{\mathfrak{m} \vee} \rightarrow \mathcal{O} .
$$

- The induced pairing

$$
\langle\cdot, \cdot\rangle_{U, \mathfrak{m}}: S(U, k)_{\mathfrak{m}} \times S(U, k)_{\mathfrak{m} \vee} \rightarrow k
$$

vanishes on restriction to the subspace $S\left(U_{1}, k\right)_{\mathfrak{m}} \times S\left(U_{1}, k\right)_{\mathfrak{m} \vee}$. (Note that for any subgroup $V \subset U_{1}$, there are isomorphisms

$$
S(V, \mathcal{O}) \otimes_{\mathcal{O}} k \cong S(V, k)
$$

compatible with the action of Hecke operators.) 
Proof. For the first part, we decompose

$$
S(V, \mathcal{O}) \otimes_{\mathcal{O}} \overline{\mathbb{Q}}_{l} \cong \bigoplus_{\sigma}\left(\iota^{-1} \sigma^{\infty}\right)^{V}
$$

A separation of eigenvalues argument shows that if $f \in\left(\iota^{-1} \sigma^{\infty}\right)^{V}, g \in\left(\iota^{-1}\left(\sigma^{\prime}\right)^{\infty}\right)^{V}$, then $\langle f, g\rangle_{V}=0$ unless $\sigma^{\prime} \cong \sigma^{\vee}$. The claim of the proposition easily follows from this statement.

For the second part, let $i: S\left(U_{1}, k\right) \rightarrow S(U, k)$ denote the natural inclusion. This can be identified with the trivial Hecke operator for the pair of subgroups $U \subset U_{1}$, and so for any $f, g \in S\left(U_{1}, k\right)$ we find the formula

$$
\langle i f, i g\rangle_{U}=\left\langle f, i^{*} i g\right\rangle_{U_{1}}
$$

where $i^{*}$ denotes adjoint with respect to the different dualities. An easy calculation (cf. [Tay89, Lemma 2]) shows that $i^{*} i$, viewed as endomorphism of $S\left(U_{1}, k\right)$, is multiplication by $\left[U_{1}: U\right]=\left[\mathfrak{P}_{v_{0}}: \mathfrak{B}_{v_{0}}\right]=q_{v_{0}}+1 \equiv$ $0 \bmod \lambda$. Restricting to the given subspace gives the desired result.

We now come to the proof of Theorem 4.1. Suppose for contradiction that there are no relevant automorphic representations $\pi$ such that $\pi_{v_{0}}$ is an unramified twist of the Steinberg representation. The space $\mathcal{M}=S(U, \mathcal{O})_{\mathfrak{m}}$ receives commuting actions of the Iwahori-Hecke algebras $H_{\mathfrak{B}_{v_{0}}, \mathcal{O}}, \ldots, H_{\mathfrak{B}_{v_{s}}, \mathcal{O}}$. By Corollary 2.10, it admits a direct sum decomposition

$$
\mathcal{M}=\bigoplus_{\mathbf{d}^{\prime} \in \prod_{i=1}^{s}\left\{X_{i}, Y_{i}\right\}} \mathcal{M}\left(\mathbf{d}^{\prime}\right)
$$

each summand being characterized by the equality (the first sum running over relevant $\pi$ ):

$$
\mathcal{M}\left(\mathbf{d}^{\prime}\right) \otimes_{\mathcal{O}} \overline{\mathbb{Q}}_{l}=\bigoplus_{\pi} \bigoplus_{\substack{\sigma \\ \text { BC }(\sigma)=\pi \\ \sigma_{v_{i}} \cong d_{i}^{\prime}, i=1, \ldots, s}}\left(\iota^{-1} \sigma^{\infty}\right)^{\text {ord }, U} .
$$

By choice of $\mathbf{d}$, if $\sigma$ appears in the decomposition of $\mathcal{M}(\mathbf{d}) \otimes_{\mathcal{O}} \overline{\mathbb{Q}}_{l}$ and the base change of $\sigma$ is not cuspidal, then $\sigma_{v_{0}} \cong X_{0}$. In particular, there is an isotypic decomposition of $H_{\mathfrak{B}_{v_{0}}, \mathbb{C} \text {-modules }}$

$$
\mathcal{M}(\mathbf{d}) \otimes_{\mathcal{O}, \iota} \mathbb{C} \cong\left(X_{0}^{\mathfrak{B}_{v_{0}}}\right)^{a} \oplus\left(Y_{0}^{\mathfrak{B}_{v_{0}}}\right)^{b},
$$

where $a>b$. Indeed, $a, b$ can be calculated as follows. For each relevant automorphic representation $\pi$, let $\sigma(\pi)=\iota^{-1} \sigma(\mathbf{d})^{v_{0} l \infty} \otimes \bigotimes_{v \mid l} \iota^{-1} \sigma(\mathbf{d})_{v}^{\text {ord }}$, an admissible representation of $G\left(\mathbb{A}_{F}^{v_{0} l \infty}\right)$, where $\sigma(\mathbf{d})$ is as in Corollary 4.4. We have

$$
a=\sum_{\pi} \operatorname{dim} \sigma(\pi)^{U^{v_{0} l}}, b=\sum_{\pi \text { cuspidal }} \operatorname{dim} \sigma(\pi)^{U^{v_{0} l}} .
$$

A priori, one need only have $a \geq b$, strict inequality occurring only when there are endoscopic representations contributing to the sum. This is the case here, due to our assumption on the existence of $\sigma_{0}$.

Let $M=\mathcal{M}(\mathbf{d})$. We define $\mathcal{N}=S(U, \mathcal{O})_{\mathfrak{m}} \vee$ and $N=\mathcal{N}(\mathbf{d})$ in an analogous manner. Let $M_{1}=$ $M^{U_{1}} \subset M$, and $N_{1}=N^{U_{1}} \subset N$. The perfect duality $\langle\cdot, \cdot\rangle_{U, \mathfrak{m}}$ restricts to a perfect duality $\langle\cdot, \cdot\rangle: M \times N \rightarrow \mathcal{O}$ satisfying the relation $\langle h x, y\rangle=\langle x, \jmath(h) y\rangle$ for all $h \in H_{\mathfrak{B}_{v_{0}}, \mathcal{O}}, x \in M, y \in N$. By Proposition 4.5 the induced perfect duality $M \otimes_{\mathcal{O}} k \times N \otimes_{\mathcal{O}} k \rightarrow k$ vanishes on the subspace $M_{1} \otimes_{\mathcal{O}} k \times N_{1} \otimes_{\mathcal{O}} k$.

We recall the abelian subalgebra $\mathcal{O}[\Lambda] \subset H_{\mathfrak{B}_{v_{0}}, \mathcal{O}}$, cf. $\{2.1$. If $\bar{\eta}: \mathcal{O}[\Lambda] \rightarrow k$ is a character of this algebra, we write $M(\bar{\eta})$ for its generalized eigenspace, i.e. the localization at ker $\bar{\eta}$ as $\mathcal{O}[\Lambda]$-module. Given a homomorphism $\bar{\eta}: \mathcal{O}[\Lambda] \rightarrow k$ we obtain a new homomorphism $\bar{\eta} \jmath: \jmath \mathcal{O}[\Lambda] \rightarrow k$, and the pairing restricts to a perfect pairing $\langle\cdot, \cdot\rangle_{\bar{\eta}}: M(\bar{\eta}) \times N(\bar{\eta} \jmath) \rightarrow \mathcal{O}$, where we write $N(\bar{\eta} \jmath)$ for the generalized $\bar{\eta} \jmath$-eigenspace of $\jmath \mathcal{O}[\Lambda]$.

By Theorem 2.7, the characters of $K[\Lambda]$ appearing amongst the Jordan-Hölder constituents of $M \otimes_{\mathcal{O}}$ $K$ as $K[\Lambda]$-module are amongst the characters

$[1, n-3, n-5, \ldots, 1],[n-3, \ldots, n-1-2 i, 1, n-3-2 i, \ldots, 1], i=1, \ldots, n / 2-2$, and $[n-3, n-5, \ldots, 1,-1]$. 
These all arise from characters $\mathcal{O}[\Lambda] \rightarrow \mathcal{O}$, and the last of these, the character $[n-3, n-5, \ldots, 1,-1]$, has distinct reduction modulo $\lambda$ from the others. Write $\bar{\eta}_{0}$ for the character $\mathcal{O}[\Lambda] \rightarrow k$ arising from reduction modulo $\lambda$ of this character. Then $M\left(\bar{\eta}_{0}\right)$ is a direct summand $\mathcal{O}[\Lambda]$-submodule of $M$ and (in the notation of Proposition 2.9. with $X=X_{0}$ and $\left.\mathfrak{B}=\mathfrak{B}_{v_{0}}\right) X_{\mathcal{O}}^{\mathfrak{B}}=X_{\mathcal{O}}^{\mathfrak{B}}\left(\bar{\eta}_{0}\right)$.

Let $M_{X}$ denote the intersection of $M$ with the $X_{0}$-isotypic piece of $M \otimes_{\mathcal{O}, \iota} \mathbb{C}$. Thus $M_{X} \subset M$ is a finite free $\mathcal{O}$-module of rank $a$, and $M / M_{X}$ is $\mathcal{O}$-torsion free. We have $M_{X} \subset M_{1}$, by Theorem 2.7 applied at the place $v_{0}$, which shows that $X_{0}^{\mathfrak{B}_{v_{0}}}=X_{0}^{\mathfrak{P}_{v_{0}}}$. Defining $N_{X} \subset N$ in the same manner, we have $N_{X} \subset N_{1}$ and $N_{X}$ is a finite free $\mathcal{O}$-module of rank $a$. Moreover, we have $M_{X}=M_{X}\left(\bar{\eta}_{0}\right)$. We also have $N_{X}=N_{X}\left(\bar{\eta}_{0} \jmath\right)$, since $X_{\mathcal{O}}^{\mathfrak{B}}=X_{\mathcal{O}}^{\mathfrak{B}}\left(\bar{\eta}_{0} \jmath\right)$, by Proposition 2.8 .

We now see that the perfect pairing

$$
\langle\cdot, \cdot\rangle_{\bar{\eta}_{0}}: M\left(\bar{\eta}_{0}\right) \otimes_{\mathcal{O}} k \times N\left(\bar{\eta}_{0} \jmath\right) \otimes_{\mathcal{O}} k \rightarrow k
$$

vanishes on the subspace $M_{X} \otimes_{\mathcal{O}} k \times N_{X} \otimes_{\mathcal{O}} k$. By construction, $M\left(\bar{\eta}_{0}\right) \otimes_{\mathcal{O}} k$ has dimension $a+b$ as a $k$-vector space, and the subspaces $M_{X} \otimes_{\mathcal{O}} k, N_{X} \otimes_{\mathcal{O}} k$ have dimension $a$. Since they annihilate each other, we must therefore have $a \leq b$. This contradicts the assumption above that $a>b$, and this contradiction completes the proof of the theorem.

\section{Construction of a special automorphic representation}

Let $E$ be an imaginary CM field with maximal totally real subfield $F$, and let $\pi$ be a RACSDC automorphic representation of $\mathrm{GL}_{2}\left(\mathbb{A}_{E}\right)$ of weight zero. Let $l \geq 5$ be prime, and let $n=l+1$. Fix an isomorphism $\iota: \overline{\mathbb{Q}}_{l} \cong \mathbb{C}$. In order to reduce notation, we now write $\rho=r_{\iota}(\pi)$. We suppose that the following hypotheses are in effect.

- For each place $v \mid l$ of $F, v$ is split in $E$ and $\left[F_{v}: \mathbb{Q}_{l}\right]$ is even. Moreover, $\pi$ is $\iota$-ordinary.

- The residual representation $\bar{\rho}: G_{E} \rightarrow \mathrm{GL}_{2}\left(\overline{\mathbb{F}}_{l}\right)$ is irreducible, and its image contains $\mathrm{SL}_{2}\left(\mathbb{F}_{l^{a}}\right)$ up to conjugation for some $a>1$. (This assumption will be used to ensure that certain symmetric powers of $\bar{\rho}$ are irreducible, and furthermore that their images are adequate, in the sense of Tho12.)

- The $(l-2)^{\text {th }}$ symmetric power of $\pi$ exists, in the following sense: there exists a RACSDC automorphic representation $\pi_{l-1}$ of $\mathrm{GL}_{l-1}\left(\mathbb{A}_{E}\right)$ such that $r_{\iota}\left(\pi_{l-1}\right) \cong \operatorname{Sym}^{l-2} \rho$.

- Let $y_{0}, \ldots, y_{s}$ denote the places of $F$ ramified in $E$. Then $s \geq 1$. Let $z_{i}$ denote the place of $E$ above $y_{i}$. For each $i=0, \ldots, s, \pi_{z_{i}} \cong \mathrm{St}_{2, E_{z_{i}}}, \bar{\rho}$ is ramified at $z_{i}$, and $q_{z_{i}}$ is a primitive element modulo $l$, and is odd.

- There exists an everywhere unramified totally real quadratic extension $F^{\prime} / F$, linearly disjoint over $F$ from the extension of $E\left(\zeta_{l}\right)$ cut out by $\bar{\rho}$, in which each place $y_{0}, \ldots, y_{s}$ is inert. We write $\omega_{F^{\prime}} / F$ for the corresponding quadratic character of $G_{F}$.

- If $w \neq z_{0}, \ldots, z_{s}$ is a place of $E$ at which $\pi$ is ramified, then $w$ is split over $F$.

Let $\chi=\operatorname{det} \rho$, and let $\varphi$ denote a continuous automorphism of $\overline{\mathbb{Q}}_{l}$ lifting the arithmetic Frobenius. There is an isomorphism (cf. [CT, §4]):

$$
\left(\operatorname{Sym}^{l} \bar{\rho}\right)^{\mathrm{ss}} \cong \varphi \bar{\rho} \oplus \bar{\chi} \otimes \operatorname{Sym}^{l-2} \bar{\rho} .
$$

In this section we will prove the following result.

Theorem 5.1. There exists a soluble $C M$ extension $M / E$, linearly disjoint over $E$ from the extension of $E\left(\zeta_{l}\right)$ cut out by $\bar{\rho}$, and a RACSDC automorphic representation $\Pi$ of $\mathrm{GL}_{n}\left(\mathbb{A}_{M}\right)$ satisfying the following:

1. $\Pi$ is ı-ordinary of weight zero.

2. There is an isomorphism $\left.\overline{r_{\iota}(\Pi)} \cong\left(\operatorname{Sym}^{l} \bar{\rho}\right)^{s s}\right|_{G_{M}}$.

3. There exists a place $w$ of $M$ above $z_{0}$ such that $\Pi_{w}$ is an unramified twist of the Steinberg representation.

Let $G$ denote the (unique up to isomorphism) unitary group in 2 variables attached to the extension $E / F$ which is quasi-split at all finite places and compact at infinity. By Proposition 3.10 and strong base change for $G$ (see, for example, Rog90, Ch. 11] and Lab11a, Théorème 5.9]), we can find a RACSDC automorphic representation $\pi_{2}$ of $\overline{G L}_{2}\left(\mathbb{A}_{E}\right)$ satisfying the following: 
- $\pi_{2}$ is $\iota$-ordinary. Writing $\rho^{\prime}=r_{\iota}\left(\pi_{2}\right)$, we have $\operatorname{HT}_{\tau}\left(\rho^{\prime}\right)=\{(1-l) / 2,(1+l) / 2\}$ for every embedding $\tau: E \hookrightarrow \overline{\mathbb{Q}}_{l}$.

- For each $i=0, \ldots, s, \pi_{2, z_{i}}$ has an Iwahori-fixed vector.

- $\bar{\rho}^{\prime} \cong \epsilon^{(l-1) / 2} \otimes \bar{\rho}$, where $\epsilon$ denotes the cyclotomic character.

- If $\pi_{2}$ is ramified at a place $w \neq z_{0}, \ldots, z_{s}$ of $E$, then $w$ is split over $F$.

Then the representation $\pi_{l+1}={ }^{\varphi} \pi_{2} \boxplus \pi_{l-1} \otimes \iota \epsilon \chi$ is conjugate self-dual and regular algebraic of weight zero, and we have

$$
r_{\iota}\left(\pi_{l+1}\right)=\epsilon^{(1-l) / 2} \otimes{ }^{\varphi} \rho^{\prime} \oplus \chi \otimes \operatorname{Sym}^{l-2} \rho .
$$

In particular, the reduction modulo $l$ of this representation is the same as that of $\operatorname{Sym}^{l} \rho$. We claim that for each $i=0, \ldots, s$ there is an isomorphism $\pi_{l+1, z_{i}} \cong \mathrm{St}_{2, E_{z_{i}}}\left(\omega_{F^{\prime} / F}\right) \boxplus \mathrm{St}_{l-1, E_{z_{i}}}$. In fact, it is easy to see that we have $\pi_{l+1, z_{i}} \cong \mathrm{St}_{2, E_{z_{i}}}\left(\psi_{2}\right) \boxplus \mathrm{St}_{l-1, E_{z_{i}}}\left(\psi_{l-1}\right)$ for some unramified characters $\psi_{2}, \psi_{l-1}$. Since $\pi_{l+1, z_{i}}$ is conjugate self-dual, these characters must be quadratic; we must show that $\psi_{2}$ is non-trivial, while $\psi_{l-1}$ is trivial. This can be done by looking, exactly as in the proof of Proposition 4.2, at the eigenvalues of a Frobenius lift at the place $z_{i}$.

Now let $L$ denote the extension of $E\left(\zeta_{l}\right)$ cut out by $\bar{\rho}$. We may choose a set $S_{1}$ of places of $E$ such that every place of $F$ below a place of $S_{1}$ is split in $F^{\prime}$, and any finite extension $E^{\prime} / E$ which is $S_{1}$-split is linearly disjoint over $E$ from $L$. We can moreover assume that $\pi$ and $L$ are unramified above $S_{1}$ (see BLGGT, Lemma A.2.2]). Let $S_{0}$ denote the set of places of $F$ below a place of $S_{1}$. Let $b$ denote the least positive integer such that $q_{z_{0}}^{b} \equiv-1 \bmod l$, and choose a cyclic totally real extension $F_{1} / F$ of degree $b$ which is $S_{0}$-split and in which $y_{0}$ is inert and each place $y_{1}, \ldots, y_{s}$ splits. (This is possible, by the Grunwald-Wang theorem, since $q_{z_{0}}$ is odd.) We write $v_{0}$ for the place of $F_{1}$ above $y_{0}$ and $v_{1}, \ldots, v_{b s}$ for the places of $F_{1}$ above $y_{1}, \ldots, y_{s}$. Let $E_{1}=E \cdot F_{1}$, and let $w_{i}$ denote the place of $E_{1}$ above $v_{i}$. Let $\pi_{l+1}^{\prime}$ denote the base change of $\varphi_{2} \otimes \omega_{F^{\prime} / F} \boxplus \pi_{l-1} \otimes \iota \epsilon \chi$ to $E_{1}$. Then $\pi_{l+1}^{\prime}$ is regular algebraic and conjugate self-dual. Moreover, for each $i=0, \ldots, b s$, we have $\pi_{l+1, w_{i}}^{\prime} \cong \mathrm{St}_{2, E_{1, w_{i}}} \boxplus \mathrm{St}_{l-1, E_{1, w_{i}}}$. We can therefore apply Theorem 4.1 to $\pi_{l+1}^{\prime}$ to deduce the existence of an automorphic representation $\Pi^{\prime}$ of $\mathrm{GL}_{n}\left(\mathbb{A}_{E_{1}}\right)$ such that $\Pi^{\prime}$ is $\iota$-ordinary of weight zero, $\Pi_{w_{0}}^{\prime}$ is an unramified twist of the Steinberg representation, and

$$
\left.\overline{r_{\iota}\left(\Pi^{\prime}\right)} \cong\left(\omega_{F^{\prime} / F} \otimes \varphi_{\bar{\rho}} \oplus \bar{\chi} \otimes \operatorname{Sym}^{l-2} \bar{\rho}\right)\right|_{G_{E_{1}}} .
$$

Now let $M=E_{1} \cdot F^{\prime}$. Then $M / E$ is a soluble extension, and $S_{1}$-split. Let $\Pi$ denote the base change of $\Pi^{\prime}$ to $M$. Then $\Pi$ is a RACSDC automorphic representation of $\mathrm{GL}_{n}\left(\mathbb{A}_{M}\right)$ which is $\iota$-ordinary of weight zero, such that $\Pi_{w}$ is an unramified twist of the Steinberg representation for any place $w$ of $M$ above $w_{0}$, and such that

$$
\left.\overline{r_{\iota}(\Pi)} \cong\left(\varphi \bar{\rho} \oplus \bar{\chi} \otimes \operatorname{Sym}^{l-2} \bar{\rho}\right)\right|_{G_{M}} .
$$

This completes the proof of Theorem 5.1 .

\section{Proof of Theorem 1.2}

In this section we prove the theorem from the introduction. Fix throughout this section a prime $l \geq 5$ and an isomorphism $\iota: \overline{\mathbb{Q}}_{l} \cong \mathbb{C}$, and a finite Galois extension $K / \mathbb{Q}$. We assume throughout this section the following hypothesis:

Conjecture 6.1 $\left(\mathrm{SP}_{l-1}\left(K\left(\zeta_{l}\right)\right)\right)$. Let $F$ be a totally real number field, linearly disjoint over $\mathbb{Q}$ from $K\left(\zeta_{l}\right)$. Let $(\pi, \chi)$ be a RAESDC automorphic representation of $\mathrm{GL}_{2}\left(\mathbb{A}_{F}\right)$ without $C M$. Then the $(l-2)^{t h}$ symmetric power lifting exists, in the following sense: there exists an RAESDC automorphic representation $\left(\pi_{l-1}, \chi_{l-1}\right)$ of $\mathrm{GL}_{l-1}\left(\mathbb{A}_{F}\right)$ and an isomorphism

$$
\operatorname{Sym}^{l-2} r_{\iota}(\pi) \cong r_{\iota}\left(\pi_{l-1}\right) .
$$

We must show that $\mathrm{SP}_{l+1}\left(K\left(\zeta_{l}\right)\right)$ holds. We begin by proving a special case, using the results accumulated above. We will reduce the general case to this one. 
Theorem 6.2. Let $F$ be a totally real number field. Let $(\pi, \chi)$ be a RAESDC automorphic representation of $\mathrm{GL}_{2}\left(\mathbb{A}_{F}\right)$, and suppose that the following hypotheses hold:

$-\pi$ is ı-ordinary of weight zero.

- Let $\rho=r_{\iota}(\pi)$. Then the residual representation $\bar{\rho}: G_{F} \rightarrow \mathrm{GL}_{2}\left(\overline{\mathbb{F}}_{l}\right)$ is irreducible, and its image contains $\mathrm{SL}_{2}\left(\mathbb{F}_{l^{a}}\right)$, up to conjugation, for some $a>1$.

- There exists a place $v$ of $F$ such that $\pi_{v}$ is an unramified twist of the Steinberg representation and $q_{v}$ is a primitive root mod $l$. Moreover, $\bar{\rho}$ is ramified at $v$.

Then $\operatorname{Sym}^{l} \rho$ is automorphic.

Proof. Let $F_{0} / F$ denote a totally real quadratic extension in which $v$ is inert, and let $\omega_{F_{0} / F}: G_{F} \rightarrow \overline{\mathbb{Q}}_{l}^{\times}$ denote the corresponding quadratic character. Let $E / F$ be a CM imaginary quadratic extension which is ramified at $v$, and in which every place of $F$ dividing $l$ splits. By [CHT08, Lemma 4.1.4] we can find an algebraic character $\psi: G_{E} \rightarrow \overline{\mathbb{Q}}_{l}^{\times}$, unramified above $v$, such that $\psi \psi^{c}=\left.r_{\iota}(\chi)\right|_{G_{E}}$. Let $F_{1} / F$ be a soluble extension satisfying the following:

- The place $v$ splits in $F_{1}$.

- Let $E_{1}=E \cdot F_{1}$. If $w \nmid v$ is a place of $E_{1}$ at which $\pi_{E}$ or $\psi_{E}$ is ramified, then $w$ is split over $F_{1}$.

- The extension $E_{1} / F_{1}$ is unramified away from places dividing $v$. The extension $F_{1} \cdot F_{0} / F_{1}$ is everywhere unramified.

- For each place $v \mid l$ of $F_{1}$, the local degree $\left[F_{1, v}: \mathbb{Q}_{l}\right]$ is even.

By choosing these extensions so that certain auxiliary primes split, we can force $F_{0} \cdot E_{1}$ to be disjoint over $F$ from the extension of $F\left(\zeta_{l}\right)$ cut out by $\bar{\rho}$ (see [BLGGT, Lemma A.2.2]). The hypotheses of $\$ 5$ are now satisfied, either for the automorphic representation $\left(\pi \otimes \iota \psi^{-1}\right)_{E_{1}}$ of $\mathrm{GL}_{2}\left(\mathbb{A}_{E_{1}}\right)$, or its twist $\left(\pi \otimes \iota \psi^{-1} \omega_{F_{0} / F}\right)_{E_{1}}$. (Since the representation $\left(\pi \otimes \iota \psi^{-1}\right)_{E_{1}}$ is conjugate self-dual, its local component at a prime $w$ dividing $v$ is isomorphic either to $\mathrm{St}_{2, E_{1, w}}$ or its twist by the quadratic unramified character of $E_{1, w}^{\times}$.) We may assume without loss of generality that it is the former. We deduce by Theorem 5.1 the existence of a soluble CM extension $M / E_{1}$ disjoint over $E_{1}$ from the extension of $E_{1}\left(\zeta_{l}\right)$ cut out by $\bar{\rho}$, and an automorphic representation $\Pi$ of $\mathrm{GL}_{l+1}\left(\mathbb{A}_{M}\right)$ such that $\Pi$ is $\iota$-ordinary, such that for some place $w$ of $M$ above $v, \Pi_{w}$ is an unramified twist of the Steinberg representation, and such that

$$
\left.\overline{r_{\iota}(\Pi)} \cong\left(\operatorname{Sym}^{l}\left(\bar{\rho} \otimes \bar{\psi}^{-1}\right)\right)\right|_{G_{M}} ^{\mathrm{ss}} .
$$

We claim that the hypotheses of [Tho, Theorem 7.1] now apply, and thus $\left.\operatorname{Sym}^{l} \rho \otimes \psi^{-1}\right|_{G_{M}}$ is automorphic. Indeed, it remains to check the following points:

- The irreducible constituents of $\left.\left(\operatorname{Sym}^{l} \bar{\rho}\right)^{\mathrm{ss}}\right|_{G_{M\left(\zeta_{l}\right)}}$ are adequate, in the sense of [Tho12, §3].

- The extension $M\left(\zeta_{l}\right)$ is not contained in the extension of $M$ cut out by ad $\bar{\rho}$.

The first point follows from our hypothesis on the image of $\bar{\rho}$ and [Gur, Theorem 1.5]. For the second point, we note that by construction we have $M \cap \mathbb{Q}\left(\zeta_{l}\right)=\mathbb{Q}$, while the image of ad $\bar{\rho}$ contains a simple normal subgroup of index at most 2. It now follows by soluble descent (see [BLGHT11, Lemma 1.4] and [BLGHT11, Lemma 1.5]) that $\mathrm{Sym}^{l} \rho$ is automorphic, and this completes the proof.

We now reduce the general case of $\mathrm{SP}_{l+1}\left(K\left(\zeta_{l}\right)\right)$ to the above one using a chain of congruences. The arguments are similar to those of [CT, §5], but since the hypotheses of the above theorem are more stringent we work a little harder. We begin by fixing a totally real field $F$, linearly disjoint over $\mathbb{Q}$ from $K\left(\zeta_{l}\right)$, and a RAESDC automorphic representation $(\pi, \chi)$ of $\mathrm{GL}_{2}\left(\mathbb{A}_{F}\right)$ without CM. Arguing as in the proof of [CT, Proposition 5.3], we can assume (after replacing $F$ by a soluble extension and passing to a congruent automorphic representation) that there is a finite set $T$ of places of $F$, a place $u$ of $F$ not in $T$, and that $\pi$ satisfies the following:

1. $\pi$ is unramified outside $T \cup\{u\}$.

2. $\pi$ is of weight zero.

3. For each place $v \mid l, \pi_{v}$ is an unramified twist of the Steinberg representation (and hence $\pi$ is $\iota$-ordinary).

4. There exists a rational prime $t>l^{2}$ such that $q_{u} \equiv-1 \bmod t$ and

$$
\left.r_{\iota}(\pi)\right|_{I_{F_{u}}} \cong\left(\begin{array}{cc}
\psi_{u} & 0 \\
0 & \psi_{u}^{q_{u}}
\end{array}\right)
$$


where $\psi_{u}: I_{F_{u}} \rightarrow \overline{\mathbb{Q}}_{l}^{\times}$is a character of order $t$. Moreover, the place $u$ is split in the maximal abelian extension of $F$ of exponent 2 which is unramified away from $T$. (We note that this implies that the representation $\left.r_{\iota}(\pi)\right|_{G_{F_{u}}}$ is absolutely irreducible, even after reduction modulo $l$.)

Fix an open compact subgroup $U \subset \mathrm{GL}_{2}\left(\mathbb{A}_{F}^{\infty}\right)$ such that $\left(\pi^{\infty}\right)^{U} \neq 0$, and let $\pi_{1}, \ldots, \pi_{n}$ denote the RAESDC automorphic representations of $\mathrm{GL}_{2}\left(\mathbb{A}_{F}\right)$ such that $\left(\pi_{i}^{\infty}\right)^{U} \neq 0$ and $\pi_{i}$ satisfies the above conditions. We can assume after renumbering that $\pi_{1}=\pi$.

For each $i=1, \ldots, n$, the residual representation $\overline{r_{\iota}\left(\pi_{i}\right)}$ is irreducible, and its image contains $\mathrm{SL}_{2}\left(\mathbb{F}_{l^{a}}\right)$ up to conjugation, for some $a>1$. This follows from an argument of Khare-Wintenberger, as follows. Let us write $\bar{\rho}=\overline{r_{\iota}\left(\pi_{i}\right)}$. Since $t>l^{2}$, the projective image of $\bar{\rho}$ contains an element of order $t>5$. The classification of finite subgroups of $\mathrm{PGL}_{2}\left(\overline{\mathbb{F}}_{l}\right)$ (see, for example, Suz82, Ch. 3, $\left.\S 6\right]$ ) implies that the projective image of $\bar{\rho}$ is conjugate either to $\mathrm{PSL}_{2}\left(\mathbb{F}_{l^{a}}\right)$ or $\mathrm{PGL}_{2}\left(\mathbb{F}_{l^{a}}\right)$, or to a dihedral subgroup. In the first case, we must have $a>1$ since $t>l^{2}$, by hypothesis. If the projective image is dihedral, then there exists a quadratic extension $M / F$ and a continuous character $\alpha: G_{M} \rightarrow \overline{\mathbb{F}}_{l}^{\times}$such that $\bar{\rho} \cong \operatorname{Ind}_{M}^{F} \alpha$. Since $\bar{\rho}$ is totally odd, $M$ is totally imaginary.

If the extension $M / F$ is ramified at a place $y$ of $F$, then $\bar{\rho}$ and hence $\pi$ is ramified at $y$, and so $y \in T \cup\{u\}$. In fact, we have $y \in T$, since $\bar{\rho}\left(I_{F_{u}}\right)$ has order $t$, prime to 2 . Thus $M / F$ is unramified outside $T$, and the place $u$ splits in $M$. This implies that the representation $\left.\bar{\rho}\right|_{G_{F_{u}}}$ is reducible, a contradiction.

Proposition 6.3. There exist a prime $p>2(l+2)$, an isomorphism $\iota_{p}: \overline{\mathbb{Q}}_{p} \cong \mathbb{C}$, and an automorphic representation $\pi^{\prime}$ satisfying conditions $2-4$ above, as well as the following conditions:

$-\overline{r_{\iota_{p}}\left(\pi^{\prime}\right)} \cong \overline{r_{\iota_{p}}(\pi)}$.

- The image of the residual representation $\overline{r_{\iota}\left(\pi^{\prime}\right)}$ contains $\mathrm{SL}_{2}\left(\mathbb{F}_{l^{a}}\right)$ up to conjugation, for some $a>1$.

- There exists a place $v$ of $F$ such that $q_{v}$ is odd and is a primitive root modulo $l$ and $\pi_{v}^{\prime}$ is an unramified twist of the Steinberg representation, and the restriction of $r_{\iota}\left(\pi^{\prime}\right)$ to $G_{F_{v}}$ is ramified.

- The symmetric $l^{\text {th }}$ power lifting of $\pi$ exists if and only if the symmetric $l^{\text {th }}$ power lifting of $\pi^{\prime}$ exists.

Proof. We construct $\pi^{\prime}$ by raising the level from $\pi$, modulo $p$. To ease notation, let us write $\overline{\rho_{i}}=\overline{r_{\iota}\left(\pi_{i}\right)}$ for $i=1, \ldots, n$. Choose a prime $p>t$ and an isomorphism $\iota_{p}: \overline{\mathbb{Q}}_{p} \cong \mathbb{C}$ such that the image of $\frac{\bar{r}_{\iota_{p}}(\pi)}{{ }^{\prime}}$ contains $\mathrm{SL}_{2}\left(\mathbb{F}_{p}\right)$ up to conjugation, and set $\bar{\rho}_{0}=\overline{r_{\iota p}(\pi)}$. We say that one of the representations $\bar{\rho}_{i}$ admits level

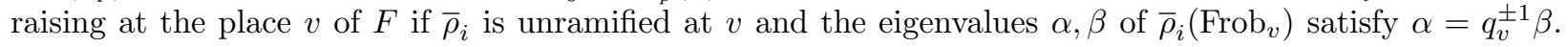
This condition depends only on the image of $\mathrm{Frob}_{v}$ under the projective representation associated to $\bar{\rho}_{i}$.

We claim that to prove the proposition, it suffices to exhibit a place $v$ of $F$ such that $q_{v}$ is odd and is a primitive root modulo $l$, and $\bar{\rho}_{0}$ admits level-raising at the place $v$ of $F$, but none of $\bar{\rho}_{1}, \ldots, \bar{\rho}_{n}$ admits level raising at $v$. Indeed, in this case we can construct using e.g. [Gee11, Corollary 3.1.7] an automorphic lift of $\bar{\rho}_{0}$ which corresponds to a RAESDC automorphic representation $\pi^{\prime}$ unramified outside $T \cup\{u, v\}$ and satisfying the desired properties, except possibly for the following:

$-\overline{r_{\iota}\left(\pi^{\prime}\right)}$ is irreducible, and its image contains $\mathrm{SL}_{2}\left(\mathbb{F}_{l^{a}}\right)$, up to conjugation, for some $a>1$.

- The restriction of $\overline{r_{\iota}\left(\pi^{\prime}\right)}$ to $G_{F_{v}}$ is ramified.

- The symmetric $l^{\text {th }}$ power lifting of $\pi$ exists if and only if the symmetric $l^{\text {th }}$ power lifting of $\pi^{\prime}$ exists.

We check that these conditions also hold. We first note that $\overline{r_{\iota}\left(\pi^{\prime}\right)}$ is, by construction, irreducible even after restriction to $G_{F_{u}}$. We claim that it is ramified after restriction to $G_{F_{v}}$. If not, then applying [Gee11, Corollary 3.1.7] once more we can find a RAESDC automorphic representation $\pi^{\prime \prime}$ which satisfies conditions 1-4 above and such that $\overline{r_{\iota}\left(\pi^{\prime \prime}\right)} \cong \overline{r_{\iota}\left(\pi^{\prime}\right)}$. Then there exists $1 \leq i \leq n$ such that $\pi^{\prime \prime}=\pi_{i}$, and this implies that $\bar{\rho}_{i}$ admits level-raising at $v$, a contradiction. In particular, the image of $\overline{r_{\iota}\left(\pi^{\prime}\right)}$ contains an element of order $l$. Since it also contains an element of order $t$, the classification of finite subgroups of $\mathrm{GL}_{2}\left(\overline{\mathbb{F}}_{l}\right)$ now shows that the image must contain a conjugate of $\mathrm{SL}_{2}\left(\mathbb{F}_{l^{a}}\right)$ for some $a>1$. (Since the image is an irreducible subgroup, it can not be contained in a Borel subgroup.) To complete the proof of the claim, we must show that the symmetric $l^{\text {th }}$ power lifting of $\pi$ exists if and only if the symmetric $l^{\text {th }}$ power lifting of $\pi^{\prime}$ exists. Since both $r_{\iota_{p}}(\pi)$ and $r_{\iota_{p}}\left(\pi^{\prime}\right)$ are potentially Barsotti-Tate, hence potentially diagonalizable, and their symmetric $l^{\text {th }}$ powers are adequate, this follows from [BLGGT, Theorem 4.2.1] (cf. the proof of [CT, Proposition 5.2]). 
We now introduce some more notation. Let $F_{i}$ denote the extension of $F$ cut out by the projective representation associated to $\bar{\rho}_{i}, i=0, \ldots, n$. Let $L$ denote the compositum of the extensions $F_{1}, \ldots, F_{n}$. Let us write $L^{\mathrm{ab}}$ and $F_{0}^{\mathrm{ab}}$ for the maximal subextensions of $L$ and $F_{0}$, respectively, which are abelian over $F$. Let $G=\operatorname{Gal}(L / F), G_{i}=\operatorname{Gal}\left(F_{i} / F\right)$. For each $i=1, \ldots, n$ there is a surjective homomorphism $p_{i}: G \rightarrow G_{i}$. The group $G_{i}$ contains a simple normal subgroup of index at most 2 , isomorphic to $\mathrm{PSL}_{2}\left(\mathbb{F}_{l^{a}}\right)$ for some $a>1$. By the Chebotarev density theorem, it now suffices to construct an element $\sigma \in \operatorname{Gal}\left(L \cdot F_{0} \cdot F\left(\zeta_{p}, \zeta_{l}\right) / F\right)$ satisfying the following conditions:

1. The projection of $\sigma$ to $\operatorname{Gal}\left(F\left(\zeta_{l}\right) / F\right)$ generates this group.

2. The projection of $\sigma$ to $\operatorname{Gal}\left(F_{0}\left(\zeta_{p}\right) / F\right)$ is trivial.

3. For each $i=1, \ldots, n$, the eigenvalues $\alpha, \beta$ (which are defined only up to scalars) of $p_{i}(\sigma)$ satisfy

$$
\alpha \neq \epsilon(\sigma)^{ \pm 1} \beta
$$

We first note that the extensions $F\left(\zeta_{l}\right)$ and $F_{0}\left(\zeta_{p}\right)$ are linearly disjoint over $F$. Indeed, $F_{0}^{\mathrm{ab}}\left(\zeta_{p}\right)$ is unramified at the primes dividing $l$. It follows that we can choose an element $\sigma_{0} \in \operatorname{Gal}\left(L \cdot F_{0} \cdot F\left(\zeta_{p}, \zeta_{l}\right) / F\right)$ satisfying the first two requirements above. We now claim that we can choose $\tau \in \operatorname{Gal}\left(L \cdot F_{0}\left(\zeta_{p}, \zeta_{l}\right) / F_{0}\left(\zeta_{p}, \zeta_{l}\right)\right)$ such that $\sigma=\tau \cdot \sigma_{0}$ satisfies all three requirements. Note that multiplying by such an element $\tau$ does not disturb the first two points.

We will in fact choose an element $\tau \in \operatorname{Gal}\left(L \cdot F_{0} \cdot F\left(\zeta_{p}, \zeta_{l}\right) / L^{\mathrm{ab}} \cdot F_{0}\left(\zeta_{p}, \zeta_{l}\right)\right)=H$, say. The group $H$ is a product of simple groups, each isomorphic to $\mathrm{PSL}_{2}\left(\mathbb{F}_{l^{a}}\right)$ for some $a>1$, and each map $\left.p_{i}\right|_{H}: H \rightarrow G_{i}$ has image of index at most 2. We show by induction on $j$ that we can choose $\tau$ such that the condition on eigenvalues is satisfied for $i=1, \ldots, j$. For the case $j=1$, we look at the image of $\sigma_{0}$ in $G_{1}$. Either the condition is satisfied for $p_{1}\left(\sigma_{0}\right) \in \mathrm{PGL}_{2}\left(\mathbb{F}_{l^{a}}\right)$ or we can choose $x \in \mathrm{PSL}_{2}\left(\mathbb{F}_{l^{a}}\right)$ such that the condition is satisfied for $p_{1}\left(\sigma_{0}\right) x$. We now take $\tau$ be be an arbitrary lift of $x$ to $H$.

For the induction step, we look at $p_{j+1}\left(\tau \sigma_{0}\right) \in \mathrm{PGL}_{2}\left(\mathbb{F}_{l^{a}}\right)$. If the condition is satisfied for this element, then we are done. If the condition is not satisfied, then the extensions $F_{j+1} \cdot L^{\mathrm{ab}}\left(\zeta_{p}, \zeta_{l}\right)$ and $F_{1} \cdots \cdots F_{j} \cdot L^{\mathrm{ab}}\left(\zeta_{p}, \zeta_{l}\right)$ are linearly disjoint over $L^{\mathrm{ab}}\left(\zeta_{p}, \zeta_{l}\right)$. For otherwise, there exists $i \in\{1, \ldots, j\}$ such that $F_{i} \cdot L^{\mathrm{ab}}\left(\zeta_{p}, \zeta_{l}\right)=F_{j+1} \cdot L^{\mathrm{ab}}\left(\zeta_{p}, \zeta_{l}\right)$; on the other hand one knows that every automorphism of $\mathrm{PSL}_{2}\left(\mathbb{F}_{l^{a}}\right)$ is a composite of conjugation by an element of $\mathrm{PGL}_{2}\left(\mathbb{F}_{l^{a}}\right)$ and the automorphism induced by a power of Frobenius, and such an automorphism does not affect the condition $\alpha \neq \epsilon(\sigma)^{ \pm 1} \beta$. We can therefore choose an element $\tau^{\prime} \in H$ such that $p_{i}(\tau)=p_{i}\left(\tau^{\prime}\right)$ for each $i=1, \ldots, j$ and $p_{j+1}\left(\tau^{\prime} \sigma_{0}\right)$ satisfies the condition on eigenvalues.

This proposition implies the result, since Theorem 6.2 now shows that the symmetric $l^{\text {th }}$ power lifting of $\pi^{\prime}$ exists. 


\section{Appendix A : Calculation of Jacquet modules}

\section{By Colette Mœglin}

\subsection{Le cas quasi déployé, introduction}

On fixe une extension quadratique $E / F$ de corps $p$-adiques; on s'intéresse au groupe $U(n, E / F)$ et $\widetilde{\mathrm{GL}}(n, E)$ est la composante non neutre qui intervient dans l'endoscopie tordue.

On écrit abusivement les induites en oubliant le parabolique mais on considère les paraboliques standard, le Borel étant les triangulaires supérieures de sorte que la représentation de Steinberg de $U(n, E / F)$ a pour module de Jacquet pour le Borel $\bigotimes_{\ell \in[(n-1) / 2,1 / 2]}||^{\ell}$.

Pour $\pi$ une représentation de $U(n, E / F)$ et pour $\chi$ un caractère de $E^{\times}$(en général une puissance d'une valeur absolue $\|^{x}$, avec $x$ demi-entier), on note $J a c_{\chi} \pi$ l'élément du groupe de Grothendieck de $U(n-$ $2, E / F)$ tel que le module de Jacquet de $\pi$ pour le parabolique maximal de Levi $E^{\times} \times U(n-2, E / F)$ est de la forme $\chi \otimes J a c_{\chi} \pi \oplus \bigoplus_{\chi^{\prime} \neq \chi, \pi^{\prime}} \chi^{\prime} \otimes \pi^{\prime}$. Pour $\tilde{\pi}$ une représentation de $\widetilde{G L}(n, E)$, on note $J a c_{\chi}^{G L} \tilde{\pi}$ la même chose sauf que l'on regarde le Levi $E^{\times} \times \widetilde{\mathrm{GL}}(n-2, E) \times E^{\times}$(on peut avoir $n=2$ mais je ne l'utiliserai pas) et le module de Jacquet est la somme de $\chi \otimes J a c_{\chi}^{\mathrm{GL}} \tilde{\pi} \otimes \bar{\chi}^{-1}$ plus d'autres termes où au moins l'une des composantes $E^{\times}$agit par un autre caractère. Comme $\tilde{\pi}$ est muni d'une action de $\theta$, de fait $J a c_{\chi}^{\mathrm{GL}}(\tilde{\pi})$ en a une aussi tout à fait canoniquement. Pour nous, cela n'interviendra pas car on évite cette difficulté.

\subsubsection{Le cas de $U(4, E)$}

Proposition. L'induite de la représentation de Steinberg de $\mathrm{GL}(2, E)$ à $U(4, E / F)$ est réductible.

Ceci résulte de [Gol93, Theorem 2.11], étant donné que cette représentation de Steinberg provient par changement de base stable de $U(2)$.

Lemme. L'induite de la proposition précédente est de longueur deux. L'un de ses sous-modules a un module de Jacquet (pour le Borel) de longueur 3; on note cette représentation $\pi_{4,+}$. L'autre représentation $\pi_{4,-} a$ un module de Jacquet irréductible. Avec des notations intuitives, le semi-simplifié du module de Jacquet de $\pi_{4,+}$ contient le terme :

$$
||^{1 / 2} \otimes||^{1 / 2}
$$

avec multiplicité 2 et le module de Jacquet de $\pi_{4,+}$ et $\pi_{4,-}$ contiennent tous deux avec multiplicité 1 le terme

$$
||^{1 / 2} \otimes||^{-1 / 2} \text {. }
$$

Le module de Jacquet de toute l'induite contient exactement les 2 termes décrits, chacun avec multiplicité 2 et chacune des sous-représentations irréductibles contient au moins avec multiplicité 1 le terme (2) par réciprocité de Frobenius. Fixons $\pi^{\prime}$, un des sous-modules irréductible dont le module de Jacquet contient avec multiplicité au moins 1 , le terme

$$
\left.\left\|\left.\right|^{1 / 2} \otimes\right\|\right|^{1 / 2}
$$

On montre qu'il contient ce terme avec multiplicité au moins $2:$ en effet on calcule le module de Jacquet de $\pi^{\prime}$ par rapport au parabolique, $P_{2}$, de Levi GL $(2, E)$. Par transitivité le calcul du module de Jacquet de $\pi^{\prime}$ par rapport au Borel se calcule en prenant d'abord le module de Jacquet par rapport au parabolique $P_{2}$ puis en passant de $\mathrm{GL}(2, E)$ au Borel de $\mathrm{GL}(2, E)$. Donc dans la première opération, on a nécessairement une représentation de $\mathrm{GL}(2, E)$ de support cuspidal ||$^{1 / 2},||^{1 / 2}$. Il n'y a qu'une représentation de $\mathrm{GL}(2, E)$ ayant cette propriété, c'est l'induite de ||$^{1 / 2} \otimes \|^{1 / 2}$ qui est irréductible. Le module de Jacquet de cette induite a bien le terme (1) de l'énoncé avec multiplicité 2. D'où le lemme. 


\subsection{Le cas de $U(n, E), n$ pair et $>4$}

\subsubsection{Nombre de séries discrètes dans le paquet}

Proposition. Il existe exactement 2 représentations elliptiques dans le paquet associé à $S t(2), S t(n-2)$ et ce sont des séries discrètes.

J'admets essentiellement cette proposition : Mœe 7.1 où ici $J o r d(\pi)$ est, par définition, l'ensemble à deux éléments (trivial, 2), (trivial, 4$)$.

\subsubsection{Rappel d'un petit lemme technique}

Lemme. Soit $\pi$ une série discrète irréductible pour $U(n, E / F)$ et $\chi$ un caractère de $E^{\times}$de la forme $\|^{x}$.

(i) $J a c_{\chi} \pi$ est soit nul soit $x>0$; si Jac $\pi \neq 0$, alors $J a c_{\chi} \pi$ est irréductible. De plus si Jac $\pi$ n'est pas nul alors $\pi$ est un sous-module irréductible de l'induite de $\chi \otimes J a c_{\chi} \pi$ pour le parabolique standard de Levi $E^{\times} \times U(n-2, E / F)$.

(ii) Soit $\pi$ et $\pi^{\prime}$ deux séries discrètes irréductibles et inéquivalentes; alors on ne peut avoir Jac $c_{\chi} \pi=$ $J a c_{\chi} \pi^{\prime}$ sauf si ces deux modules sont nuls.

En fait il y a unicité du sous-module irréductible dans (i) mais on n'en a pas besoin.

La première assertion de (i) est uniquement le critère de Casselman pour les séries discrètes : Cas Theorem 4.4.6].

Pour l'irréductibilité de (i), c'est Mœg corollaire de 2.7 (i). Montrons l'inclusion : la non nullité de $J a c_{\chi} \pi$ entraîne que le module de Jacquet de $\pi$ pour le parabolique standard de Levi isomorphe à $E^{\times} \times$ $U(n-2, E / F)$ a un quotient irréductible de la forme $\chi \otimes \sigma$; par irréductibilité de $J a c_{\chi} \pi$, nécessairement $\sigma=J a c_{\chi} \pi$. Par réciprocité de Frobenius, on a alors une inclusion de $\pi$ dans l'induite comme annoncé.

Pour (ii) c'est Mœg corollaire 2.7 (ii) avec le fait que toute série discrète est dans un paquet stable (Mœg 2.4, où n'importe quelle autre référence).

\subsubsection{Calcul des modules de Jacquet}

On peut aller plus loin, en utilisant le fait que le module de Jacquet commute au transfert. On a, pour $n \geq 6, J_{c} c_{\chi}^{\mathrm{GL}}(S t(2) \times S t(n-2))=0$ sauf exactement si $\chi=||^{1 / 2}$ ou $\chi=||^{(n-3) / 2}$. On a $J a c_{||^{1 / 2}}^{\mathrm{GL}} S t(2) \times$ $S t(n-2)=S t(n-2)$ et $J a c_{||^{(n-3) / 2}}^{\mathrm{GL}} S t(2) \times S t(n-2)=S t(2) \times S t(n-4)$ et il n'y a pas de multiplicité; donc l'action de $\theta$ est bien déterminée à un signe près, dont on se moque.

Proposition. On suppose que $n \geq 6$

(i) On suppose que $\pi$ est dans le paquet de séries discrètes associées à $S t(2), S t(n-2)$. Alors $J a c_{\chi} \pi=0$ sauf éventuellement si $\chi=||^{1 / 2}$ ou $\chi=||^{(n-3) / 2}$.

(ii) $\operatorname{Jac}_{\left.\right|_{1 / 2} ^{1 / 2}}(\pi)=0$ ou est la représentation de Steinberg de $U(n-2, E / F)$, chacun de ces deux cas se produisant pour un bon choix de $\pi$ dans le paquet; on note $\pi_{n,+}$ celle des deux représentations du paquet telle que $J a c_{\left.\right|_{1 / 2} / 2} \pi 0$ et $\pi_{n,-}$ l'autre représentation.

(iii) Avec la définition glissée dans (ii) et celle du paragraphe 6.1.1, pour tout $n \geq 6$, on a pour $\zeta= \pm, J a c_{\mid(n-3) / 2} \pi_{n, \zeta}=\pi_{n-2, \zeta}$.

Le (i) est juste la compatibilité des modules de Jacquet au transfert. Pour (ii) et (iii) on introduit les notations suivantes : soit $\pi_{i}$ pour $i=1,2$ les deux séries discrètes dans le paquet considéré. Soit $a_{i}$ des nombres complexes non nuls tels que $\sigma:=a_{1} \pi_{1}+a_{2} \pi_{2}$ est stable.

Montrons (ii) $J a c_{||^{1 / 2}} \sigma$ est une distribution stable (compatibilité de la stabilité à la restriction) et elle se transfère (à un scalaire près ) en la trace tordue de la représentation de Steinberg de GL $(n-2, E)$; donc $J a c_{\mid 1 / 2} \sigma$ est nécessairement (à un scalaire près) la représentation de Steinberg de $U(n-2, E / F)$. Par l'irréductibilité rappelée ci-dessus 6.2 .2 (i)) et le fait que $J a c_{\chi} \pi_{1} \neq J a c_{\chi} \pi_{2}$ si l'un des deux modules de Jacquet est non nul $(6.2 .2($ ii $))$, il existe exactement un $i$ tel que $J a c_{||^{1 / 2}} \pi_{i} \neq 0$ et ce module de Jacquet vaut alors la représentation de Steinberg de $U(n-2, E / F)$. 
Pour (iii), $J a c_{||(n-3) / 2}\left(a_{1} \pi_{1}+a_{2} \pi_{2}\right)$ est (via la trace) une distribution stable et elle se transfère à un scalaire près en la trace tordue de $\operatorname{Ind} S t(2) \otimes S t(n-4)$. Si $n=6$, l'induite $\operatorname{Ind} S t(2) \otimes S t(n-4)$ n'est pas $\theta$-elliptique c'est une induite à partir d'une représentation $\theta$-stable. Son caractère est le transfert de la représentation de $U(4, E / F)$, Ind $S t_{\mathrm{GL}(2, E)}(2)$ et on a calculé cette distribution; c'est le caractère de $\pi_{4,+}+\pi_{4,-}$. On montre par récurrence sur $n$ que $a_{1}=a_{2}$ et l'égalité d'ensembles non ordonnés :

$$
\left(J a c_{||^{(n-3) / 2}} \pi_{1}, J a c_{||^{(n-3) / 2}} \pi_{2}\right)=\left(\pi_{n-2,+}, \pi_{n-2},-\right) .
$$

Initialiser la récurrence avec $n=6$ se fait en même temps que le pas général.

En effet, quitte à multiplier $a_{1}$ et $a_{2}$ par le même nombre complexe non nul $J a c_{||^{(n-3) / 2}}\left(a_{1} \pi_{1}+a_{2} \pi_{2}\right)=$ $\pi_{n-2,+}+\pi_{n-2,-}$, car c'est (à un scalaire près) la distribution stable portée par $\pi_{n-2,+}$ et $\pi_{n-2,-}$ que l'on connait pour $n=6$, on vient de le rappeler, et par l'hypothèse de récurrence pour $n>6$. Le membre de gauche vaut

$$
a_{1} J a c_{||^{(n-3) / 2}} \pi_{1}+a_{2} J a c_{||^{(n-3) / 2}} \pi_{2}
$$

et chaque terme est soit nul soit une représentation irréductible, les deux ne pouvant être simultanément non nuls et égaux $6.2 .2(\mathrm{i})$ et (ii)). Ainsi $a_{1}=a_{2}=1$ et l'égalité d'ensemble non ordonné annoncée.

Supposons que $J a c_{||^{(n-3) / 2} / 2} \pi_{1}=\pi_{n-2,+}$ donc $J a c_{||^{(n-3) / 2}} \pi_{2}=\pi_{n-2,-}$. On sait $\sqrt{6.2 .2}$ (i)) que $\pi_{2} \hookrightarrow$ Ind ||$^{(n-3) / 2} \otimes \pi_{n-2,-}$.

On traite d'abord le cas de $n=6$ qui est le seul cas où $J a c_{||^{1 / 2}} \pi_{n-2,-} \neq 0$. Dans ce cas, par les formules générales de calcul de module de Jacquet, on a :

$$
J a c_{||^{1 / 2}} \operatorname{Ind}\left(||^{5 / 2} \times \pi_{4,-}\right)=\left.\operatorname{Ind}||^{5 / 2} \otimes\right|^{-1 / 2},
$$

car $J a c_{||^{1 / 2}} \pi_{4,-}=||^{-1 / 2}$ d'après la description donnée dans le paragraphe 6.1.1. Par exactitude du module de Jacquet, on a l'inclusion :

$$
J a c_{||^{1 / 2}} \pi_{6,-} \hookrightarrow \operatorname{Ind}||^{5 / 2} \otimes||^{-1 / 2} \simeq \operatorname{Ind}||^{-1 / 2} \otimes||^{5 / 2}
$$

l'isomorphisme est, par transitivité, une propriété de $\mathrm{GL}(2, E)$ et dans ce groupe l'induite de ||$^{5 / 2} \otimes||^{-1 / 2}$ est irréductible. Ainsi si $J a c_{\mid 1 / 2} \pi_{6,-}$ est non nul c'est un sous-module irréductible de l'induite Ind ||$^{-1 / 2} \otimes||^{5 / 2}$. D'où une inclusion (cf. 6.2.2 (i))

$$
\pi_{6,-} \hookrightarrow \operatorname{Ind}\left(||^{1 / 2} \otimes J a c_{||^{1 / 2}} \pi_{6,-}\right) \hookrightarrow \operatorname{Ind}||^{1 / 2} \otimes||^{-1 / 2} \otimes||^{5 / 2} .
$$

Ceci est impossible pour une série discrète car $1 / 2+(-1 / 2)=0$ et cela contredit le critère de Casselman. Ainsi $J a c_{||^{1 / 2}} \pi_{6,-}=0$.

On suppose $n>6$. Par hypothèse de récurrence $J a c_{||^{1 / 2}} \pi_{n-2,-}=0$ et $(n-3) / 2 \neq \pm 1 / 2$; donc les formules standard de calcul de module de Jacquet donnent

$$
J a c_{||^{1 / 2}} \operatorname{Ind}||^{(n-3) / 2} \otimes \pi_{n-2,-}=\operatorname{Ind}\left(||^{(n-3) / 2} \otimes J a c_{||^{1 / 2}} \pi_{n-2,-}\right)=0,
$$

par l'hypothèse de récurrence puisque $n-2 \geq 6$. Par exactitude des modules de Jacquet cela force aussi $J a c_{||^{1 / 2}} \pi_{2}=0$ donc $\pi_{2} \neq \pi_{n-2,+}$; d'où $\pi_{1}=\pi_{n-2,+}$ par (ii). Cela termine la preuve de (iii).

Corollaire. Ici on suppose $n \geq 4$.

(i) Le module de Jacquet (pour le Borel) de $\pi_{n,-}$ est de longueur 1 ; il est réduit à

$$
\bigotimes_{\ell \in[(n-3) / 2,-1 / 2]}||^{\ell}
$$

où on décale de 1 en 1 (et non $1 / 2$ )

(ii) Le module de Jacquet (pour le Borel) de $\pi_{n,+}$ est de longueur $(n-2) / 2+2$. Il contient avec multiplicité 1 le terme

$$
\bigotimes_{\ell \in[(n-3) / 2,-1 / 2]}||^{\ell}
$$


avec multiplicité 2 le terme

$$
\bigotimes_{\ell \in[(n-3) / 2,1 / 2]}||^{\ell} \otimes||^{1 / 2}
$$

et avec mutliplicité 1 tous les termes

$$
\bigotimes_{\ell \in[(n-3) / 2,1 / 2]} \|^{\ell}
$$

et où on glisse ||$^{1 / 2}$ juste à gauche de l'un des ||$^{\ell}$ avec $\ell>1 / 2$.

Le corollaire est vrai pour $n=4$ grâce au paragraphe 6.1.1. Pour $n>4$ on le démontre ainsi.

Le (i) se démontre par récurrence : on sait que $J a c_{\chi} \pi_{n,-}=0$ sauf pour $\chi=||^{(n-3) / 2}$ et $J a c_{||^{(n-3) / 2}} \pi_{n,-}=$ $\pi_{n-2,-}$. Par transitivité, le module de Jacquet pour le Borel de $\pi_{n,-}$ est le produit tensoriel de ||$^{(n-3) / 2}$ avec le module de Jacquet (pour le Borel) de $\pi_{n-2,-}$.

Pour (ii) le même argument que pour (i) calcule tous les termes du module de Jacquet de $\pi_{n,+}$ qui commence par ||$^{(n-3) / 2}$ et il faut ajouter les termes qui commencent par ||$^{1 / 2}$. Mais il n'y a en qu'un puisque $J a c_{||^{1 / 2}}=S t_{U(n-2)}$ et c'est la description de l'énoncé : le module de Jacquet de $S t_{U(n-2)}$ est évidemment égal à $\bigotimes_{\ell \in[(n-3) / 2,1 / 2]}||^{\ell}$.

\section{References}

[Art13] James Arthur. The endoscopic classification of representations, volume 61 of American Mathematical Society Colloquium Publications. American Mathematical Society, Providence, RI, 2013. Orthogonal and symplectic groups.

[BG06] Joël Bellaïche and Phillipe Graftieaux. Augmentation du niveau pour U(3). Amer. J. Math., 128(2):271-309, 2006.

[BLGGT] Thomas Barnet-Lamb, Toby Gee, David Geraghty, and Richard Taylor. Potential automorphy and change of weight. To appear in Annals of Math.

[BLGHT11] Tom Barnet-Lamb, David Geraghty, Michael Harris, and Richard Taylor. A family of CalabiYau varieties and potential automorphy II. Publ. Res. Inst. Math. Sci., 47(1):29-98, 2011.

[BMM] Nicolas Bergeron, John Millson, and Colette Moeglin. Hodge type theorems for arithmetic manifolds associated to orthogonal groups. Preprint. Available at http://arxiv.org/abs/1110.3049.

[Bor76] Armand Borel. Admissible representations of a semi-simple group over a local field with vectors fixed under an Iwahori subgroup. Invent. Math., 35:233-259, 1976.

[BT72] F. Bruhat and J. Tits. Groupes réductifs sur un corps local. Inst. Hautes Études Sci. Publ. Math., (41):5-251, 1972.

[Car79] P. Cartier. Representations of p-adic groups: a survey. In Automorphic forms, representations and L-functions (Proc. Sympos. Pure Math., Oregon State Univ., Corvallis, Ore., 1977), Part 1, Proc. Sympos. Pure Math., XXXIII, pages 111-155. Amer. Math. Soc., Providence, R.I., 1979.

[Car12] Ana Caraiani. Local-global compatibility and the action of monodromy on nearby cycles. Duke Math. J., 161(12):2311-2413, 2012.

[Cas] W. Casselman. Introduction to the theory of admissible representations of $p$ adic reductive groups. Unpublished draft of 1 May 1995. Available online at http://www.math.ubc.ca/ cass/research/pdf/p-adic- book.pdf.

[Cas80] W. Casselman. The unramified principal series of $\mathfrak{p}$-adic groups. I. The spherical function. Compositio Math., 40(3):387-406, 1980.

[CH] Gaëtan Chenevier and Michael Harris. Construction of automorphic Galois representations, II. To appear in Cambridge Math. Journal, vol. 1 (2013). 
[CHL11] Laurent Clozel, Michael Harris, and Jean-Pierre Labesse. Endoscopic transfer. In On the stabilization of the trace formula, volume 1 of Stab. Trace Formula Shimura Var. Arith. Appl., pages 475-496. Int. Press, Somerville, MA, 2011.

[CHT08] Laurent Clozel, Michael Harris, and Richard Taylor. Automorphy for some $l$-adic lifts of automorphic mod $l$ Galois representations. Publ. Math. Inst. Hautes Études Sci., (108):1-181, 2008. With Appendix A, summarizing unpublished work of Russ Mann, and Appendix B by Marie-France Vignéras.

[CL10] Pierre-Henri Chaudouard and Gérard Laumon. Le lemme fondamental pondéré. I. Constructions géométriques. Compos. Math., 146(6):1416-1506, 2010.

[CL12] Pierre-Henri Chaudouard and Gérard Laumon. Le lemme fondamental pondéré. II. Énoncés cohomologiques. Ann. of Math. (2), 176(3):1647-1781, 2012.

[Clo90a] Laurent Clozel. The fundamental lemma for stable base change. Duke Math. J., 61(1):255-302, 1990.

[Clo90b] Laurent Clozel. Motifs et formes automorphes: applications du principe de fonctorialité. In $A u-$ tomorphic forms, Shimura varieties, and L-functions, Vol. I (Ann Arbor, MI, 1988), volume 10 of Perspect. Math., pages 77-159. Academic Press, Boston, MA, 1990.

[Clo11] Laurent Clozel. Identités de caractères en la place archimédienne. In On the stabilization of the trace formula, volume 1 of Stab. Trace Formula Shimura Var. Arith. Appl., pages 351-367. Int. Press, Somerville, MA, 2011.

[CT] Laurent Clozel and Jack A. Thorne. Level raising and symmetric power functoriality, I. To appear in Compos. Math.

[Gee11] Toby Gee. Automorphic lifts of prescribed types. Math. Ann., 350(1):107-144, 2011.

[Ger] David Geraghty. Modularity lifting theorems for ordinary Galois representations. Preprint.

[Gol93] David Goldberg. Reducibility of generalized principal series representations of U $(2,2)$ via base change. Compositio Math., 86(3):245-264, 1993.

[Gur] Robert Guralnick. Adequacy of representations of finite groups of Lie type. Preprint. Appendix to a paper by L. Dieulefait.

[Hen02] Guy Henniart. Une caractérisation de la correspondance de Langlands locale pour GL(n). Bull. Soc. Math. France, 130(4):587-602, 2002.

[HT01] Michael Harris and Richard Taylor. The geometry and cohomology of some simple Shimura varieties, volume 151 of Annals of Mathematics Studies. Princeton University Press, Princeton, NJ, 2001. With an appendix by Vladimir G. Berkovich.

[Iwa66] Nagayoshi Iwahori. Generalized Tits system (Bruhat decompostition) on $p$-adic semisimple groups. In Algebraic Groups and Discontinuous Subgroups (Proc. Sympos. Pure Math., Boulder, Colo., 1965), pages 71-83. Amer. Math. Soc., Providence, R.I., 1966.

[KS99] Robert E. Kottwitz and Diana Shelstad. Foundations of twisted endoscopy. Astérisque, (255):vi+190, 1999.

[KS02] Henry H. Kim and Freydoon Shahidi. Functorial products for $\mathrm{GL}_{2} \times \mathrm{GL}_{3}$ and the symmetric cube for $\mathrm{GL}_{2}$. Ann. of Math. (2), 155(3):837-893, 2002. With an appendix by Colin J. Bushnell and Guy Henniart.

[Lab11a] J.-P. Labesse. Changement de base CM et séries discrètes. In On the stabilization of the trace formula, volume 1 of Stab. Trace Formula Shimura Var. Arith. Appl., pages 429-470. Int. Press, Somerville, MA, 2011.

[Lab11b] J.-P. Labesse. Les facteurs de transfert pour les groupes unitaires. In On the stabilization of the trace formula, volume 1 of Stab. Trace Formula Shimura Var. Arith. Appl., pages 411-421. Int. Press, Somerville, MA, 2011.

[LS87] R. P. Langlands and D. Shelstad. On the definition of transfer factors. Math. Ann., 278(14):219-271, 1987. 
[Lus89] George Lusztig. Affine Hecke algebras and their graded version. J. Amer. Math. Soc., 2(3):599635, 1989.

[Mín11] Alberto Mínguez. Unramified representations of unitary groups. In On the stabilization of the trace formula, volume 1 of Stab. Trace Formula Shimura Var. Arith. Appl., pages 389-410. Int. Press, Somerville, MA, 2011.

[Mœg] C. Møglin. Paquets stables des séries discrètes accessibles par endoscopie tordue; leur paramètre de Langlands. Available at arXiv:1212.5433 or http://www.math.jussieu.fr/ moeglin. Preprint.

[Møg07] Colette Møglin. Classification et changement de base pour les séries discrètes des groupes unitaires p-adiques. Pacific J. Math., 233(1):159-204, 2007.

[Mok] Chung Pang Mok. Endoscopic classification of representations of quasi-split unitary groups. To appear in Memoirs of the AMS.

[Mor99] Lawrence Morris. Level zero G-types. Compositio Math., 118(2):135-157, 1999.

[Ngô10] Bao Châu Ngô. Le lemme fondamental pour les algèbres de Lie. Publ. Math. Inst. Hautes Études Sci., (111):1-169, 2010.

[Ree97] Mark Reeder. Nonstandard intertwining operators and the structure of unramified principal series representations. Forum Math., 9(4):457-516, 1997.

[Ree00] Mark Reeder. Matrices for affine Hecke modules. J. Algebra, 231(2):758-785, 2000.

[Rib84] Kenneth A. Ribet. Congruence relations between modular forms. In Proceedings of the International Congress of Mathematicians, Vol. 1, 2 (Warsaw, 1983), pages 503-514, Warsaw, 1984. PWN.

[Rog90] Jonathan D. Rogawski. Automorphic representations of unitary groups in three variables, volume 123 of Annals of Mathematics Studies. Princeton University Press, Princeton, NJ, 1990.

[Suz82] Michio Suzuki. Group theory. I, volume 247 of Grundlehren der Mathematischen Wissenschaften [Fundamental Principles of Mathematical Sciences]. Springer-Verlag, Berlin, 1982. Translated from the Japanese by the author.

[Tat79] J. Tate. Number theoretic background. In Automorphic forms, representations and L-functions (Proc. Sympos. Pure Math., Oregon State Univ., Corvallis, Ore., 1977), Part 2, Proc. Sympos. Pure Math., XXXIII, pages 3-26. Amer. Math. Soc., Providence, R.I., 1979.

[Tay89] Richard Taylor. On Galois representations associated to Hilbert modular forms. Invent. Math., 98(2):265-280, 1989.

[Tho] Jack A. Thorne. Automorphy lifting for residually reducible $l$-adic Galois representations. Preprint.

[Tho12] Jack A. Thorne. On the automorphy of $l$-adic Galois representations with small residual image. Journal of the Inst. of Math. Jussieu, 11(4):855-920, 2012. With an appendix by Robert Guralnick, Florian Herzig, Richard Taylor and Jack Thorne.

[Tit79] J. Tits. Reductive groups over local fields. In Automorphic forms, representations and $L$ functions (Proc. Sympos. Pure Math., Oregon State Univ., Corvallis, Ore., 1977), Part 1, Proc. Sympos. Pure Math., XXXIII, pages 29-69. Amer. Math. Soc., Providence, R.I., 1979.

[Wala] J.-L. Waldspurger. Stabilisation de la formule des traces tordue I: endoscopie tordue sur un corps local. Preprint. Available at http://arxiv.org/abs/1401.4569.

[Walb] J.-L. Waldspurger. Stabilisation de la formule des traces tordue II: intégrales orbitales et endoscopie sur un corps local non-archimédien; définitions et énoncés des résultats. Preprint. Available at http://arxiv.org/abs/1401.7127.

[Wal97] J.-L. Waldspurger. Le lemme fondamental implique le transfert. Compositio Math., 105(2):153236, 1997.

[Wal09a] J.-L. Waldspurger. À propos du lemme fondamental pondéré tordu. Math. Ann., 343(1):103174, 2009. 
[Wal09b] Jean-Loup Waldspurger. Endoscopie et changement de caractéristique: intégrales orbitales pondérées. Ann. Inst. Fourier (Grenoble), 59(5):1753-1818, 2009. 